\title{
Three editions of the star catalogue of Tycho Brahe
}

\section{Machine-readable versions and comparison with the modern Hipparcos Catalogue $^{\star}$}

\author{
F. Verbunt ${ }^{1}$ and R. H. van Gent ${ }^{2,3}$ \\ 1 Astronomical Institute, Utrecht University, PO Box 80 000, 3508 TA Utrecht, The Netherlands \\ e-mail: f.w.m.verbunt@uu.nl \\ 2 URU-Explokart, Faculty of Geosciences, Utrecht University, PO Box 80 115, 3508 TC Utrecht, The Netherlands \\ 3 Institute for the History and Foundations of Science, PO Box 80 000, 3508 TA Utrecht, The Netherlands
}

Received 6 January 2010 / Accepted 3 February 2010

\section{ABSTRACT}

\begin{abstract}
Tycho Brahe completed his catalogue with the positions and magnitudes of 1004 fixed stars in 1598 . This catalogue circulated in manuscript form. Brahe edited a shorter version with 777 stars, printed in 1602, and Kepler edited the full catalogue of 1004 stars, printed in 1627. We provide machine-readable versions of the three versions of the catalogue, describe the differences between them and briefly discuss their accuracy on the basis of comparison with modern data from the Hipparcos Catalogue. We also compare our results with earlier analyses by Dreyer (1916, Tychonis Brahe Dani Scripta Astronomica, Vol. II) and Rawlins (1993, DIO, 3, 1), finding good overall agreement. The magnitudes given by Brahe correlate well with modern values, his longitudes and latitudes have error distributions with widths of 2', with excess numbers of stars with larger errors (as compared to Gaussian distributions), in particular for the faintest stars. Errors in positions larger than $\simeq 10^{\prime}$, which comprise about $15 \%$ of the entries, are likely due to computing or copying errors.
\end{abstract}

Key words. astrometry - history and philosophy of astronomy

\section{Introduction}

The astronomical observations of Tycho Brahe improved by an order of magnitude on the positional accuracy achieved by his predecessors. His measurements of the positions of stars on the celestial sphere resulted in a manuscript catalogue in 1598 (Brahe 1598). Astronomers and mapmakers throughout Europe used handwritten copies of this catalogue. Brahe edited a shorter version, with 777 stars, which was printed in 1602 as part of Astronomiae Instauratae Progymnasmata (Brahe 1602). The full list of 1004 entries with some modifications was published by Johannes Kepler in 1627 as part of the Tabulae Rudolphinae (Kepler 1627).

These catalogues are a monument in the history of astronomy, and as such have been studied repeatedly (e.g. Baily 1843; Dreyer 1890; Rawlins 1993).

In this paper we describe machine-readable versions of the catalogues. In addition to the numbers given by Brahe (and Kepler) the machine-readable tables provide crossreferences between the catalogues, identifications with stars from the (modern!) Hipparcos Catalogue (ESA 1997) and on the basis of these the accuracy of the positions and magnitudes tabulated in the old catalogues.

The accuracy of the measurements of Brahe is best studied by reference to his observational logs rather than by reference to his reduced data, and Wesley (1978) has shown that the measurement accuracy varies between the instruments used by Brahe. For the mural quadrant, for example, the average error is 34 "' 6 .

* The full tables KeplerE and Variants (see Table 4) and the table with the latin descriptions of the stars are available in electronic form only at the CDS via anonymous ftp to cdsarc.u-strasbg.fr $(130.79 .128 .5)$ or via

http://cdsweb.u-strasbg.fr/cgi-bin/qcat? J/A+A/516/A28
In his comprehensive study of Brahe's star catalogue, Rawlins (1993) also refers to the observational logs to correct errors that Brahe made in producing his star catalogue. An important conclusion drawn by Rawlins is that errors exceeding 6' are usually the consequence of errors in the reduction of the measurements, rather than in the measurements themselves. Such errors include repetition of stars in several entries, mixing data for different stars for one entry, and spurious entries. Rawlins (1993) produces an improved version of the star catalogue of Brahe, the best version that Brahe could have published on the basis of his measurements. The goal of our edition is to present the star catalogue of Brahe as it was available for use to a 17th century astronomer or map maker, i.e. the versions as given by Brahe and Kepler.

Our analysis improves on earlier ones in three ways. First and foremost, our analysis is based on the Hipparcos Catalogue (ESA 1997), which is more accurate, complete and homogeneous than the stellar catalogues used in earlier analyses. Second, we grade each identification, discriminating between secure, probable and merely possible. Third, we provide images of each constellation comparing the positions and magnitudes from the Brahe catalogue with the posistion and magnitudes of all stars in the field, thus illustrating which stars were selected by Brahe for measurements and which ones not.

In describing the different versions of the catalogues we use the following notation: Manuscript refers to the manuscript version (Brahe 1598), Progym refers to the version edited and printed by Brahe (1602), Kepler refers to the version edited by Kepler (1627), and KeplerE to our emended version of the latter catalogue. Individual entries are numbered according to the order in which they appear in the different versions. For example, the third entry in Kepler corresponds to entry 338 in Manuscript, which we denote as K3 and M338, respectively. 
(Thus, our $\mathrm{M}$ numbers correspond to $\mathrm{D}$ numbers of Rawlins 1993.) The sequence number within a constellation is indicated by a number following the abbreviated constellation name: Oph 14 is the 14th star of Ophiuchus.

\section{Description of the catalogues}

The star catalogue of Brahe is organized by constellation. Manuscript starts with the zodiacal constellations, followed first by the Northern and then by the Southern constellations. Brahe added two constellations with respect to those given by Ptolemaios: Coma Berenices (between Bootes and Corona Borealis), and Antinous (between Aquila and Delphinus) and omitted the southern constellations that follow Centaurus in the catalogue of Ptolemaios, viz. Lupus, Ara, Corona Australis, and Piscis Austrinus. For many constellations the list of stars belonging to it is followed by a list of additional stars near to, but not considered to be part of, the constellation. In the case of Ophiuchus and Serpens the additional stars precede the stars of the constellations. The total number of entries in Manuscript is 1004 , but as we will see below up to fourteen stars occur more than once, so that the actual number of stars present in the catalogue is closer to 990 . The version we use is the one given in Volume 3 of the complete works by Tycho Brahe as edited by Dreyer (1916). Dreyer based his edition mainly on Codex 306 in the Copenhagen Royal Library, but also consulted various other manuscripts (Dreyer 1916: p. 332).

In Progym the additional stars and occasionally some more stars at the end of the constellation have been omitted, and the constellation Centaurus is absent, reducing the total number of entries to 777. Coma Berenices is listed here between Ericthonius and Ophiuchus. No star occurs twice in this shorter catalogue, and the number of stars in it equals the number of entries. The version we use is the reprint in Volume 2 of the complete works by Tycho Brahe edited by Dreyer (1915).

Kepler restores the order of constellations as used by Ptolemaios; it also restores most stars omitted in Progym. For some stars Kepler adds an alternative position, indicated with meus catalogus ("my catalogue") or with Piferus or abbreviated versions of these indications. For this catalogue we use the orginal edition (Kepler 1627). Piferus refers to Francesco Pifferi whose translation with commentary of Sacrobosco's Tractatus de Sphaera was printed in Siena in 1604. In comments with some entries Kepler (1627) refers to Grünbergerus, i.e. Christoph Grünberger, who in 1612 brought out an edition of Brahe's star list.

The ordering of the constellations and the numbers of stars in each constellation is given for the two printed editions (1602 and 1627 ) in Table 1. Generally, Manuscript has the same stars in the same order within each constellation as Kepler. Exceptions are

- Kepler adds new stars in Cygnus (Nova $1600=$ PCygni) and Cassiopeia (Nova 1572) at the end of these constellations. (Remarakbly, the supernova of 1604, now named after Kepler, is not in the catalogue ${ }^{1}$.)

- Manuscript lists thirteen stars denoted as Sequentes pertinent ad Ophiuchum et eius Serpentem (the following belong to Ophiuchus and his Snake) before the stars of Ophiuchus. Manuscript also erroneously gives the position of the Oph 14 as identical to that of Oph 12. Kepler places these thirteen

\footnotetext{
${ }_{1}$ It is however in the Secunda Classis, a list which immediately follows the star catalogue of Brahe in Kepler's (1627) edition, and which contains stars not in the Brahe catalogue
}

Table 1. Constellations and numbers of stars in them.

\begin{tabular}{|c|c|c|c|c|c|c|}
\hline$C_{B}$ & $C$ & & $N$ & $N_{a}$ & K & Constellation \\
\hline 13 & 1 & UMi & 7 & 13 & 1 & Ursa Minor, Cynosura \\
\hline 14 & 2 & UMa & 29 & 27 & 21 & Ursa Maior, Helice \\
\hline 15 & 3 & Dra & 32 & 0 & 77 & Draco \\
\hline 16 & 4 & Cep & 4 & 7 & 109 & Cepheus \\
\hline 17 & 5 & Boo & 18 & 10 & 120 & Bootes, Arctophylax \\
\hline 18 & 6 & $\mathrm{CrB}$ & 8 & 0 & 148 & Corona Borea \\
\hline 19 & 7 & Her & 28 & 0 & 156 & Engonasi, Hercules \\
\hline 20 & 8 & Lyr & 11 & 0 & 184 & Lyra, Vultur Cadens \\
\hline 21 & 9 & Cyg & 18 & 9 & 195 & Olor, Cygnus \\
\hline 22 & 10 & Cas & 26 & 20 & 222 & Cassiopeia \\
\hline 23 & 11 & Per & 29 & 4 & 268 & Perseus \\
\hline 24 & 12 & Aur & 9 & 18 & 301 & Auriga, Heniochus \\
\hline 26 & 13 & Oph & 15 & 22 & 328 & Ophiuchus, Serpentarius \\
\hline 27 & 14 & Ser & 13 & 0 & 365 & Serpens Ophiuchi \\
\hline 28 & 15 & Sge & 5 & 3 & 378 & Sagitta sive Telum \\
\hline 29 & 16 & Aql & 12 & 0 & 386 & Aquila seu Vultur Volans \\
\hline 30 & 17 & Atn & 3 & 4 & 398 & Antinous \\
\hline 31 & 18 & Del & 10 & 0 & 405 & Delphinus \\
\hline 32 & 19 & Equ & 4 & 0 & 415 & Equuleus, Equi Sectio \\
\hline 33 & 20 & Peg & 19 & 4 & 419 & Pegasus, Equus Alatus \\
\hline 34 & 21 & And & 23 & 0 & 442 & Andromeda \\
\hline 35 & 22 & Tri & 4 & 0 & 465 & Triangulus, Deltoton \\
\hline 25 & 23 & Com & 14 & 1 & 469 & Coma Berenices \\
\hline 1 & 24 & Ari & 21 & 0 & 484 & Aries \\
\hline 2 & 25 & Tau & 43 & 0 & 505 & Taurus \\
\hline 3 & 26 & Gem & 25 & 4 & 548 & Gemini \\
\hline 4 & 27 & $\mathrm{Cnc}$ & 15 & 0 & 577 & Cancer \\
\hline 5 & 28 & Leo & 30 & 10 & 592 & Leo \\
\hline 6 & 29 & Vir & 33 & 6 & 632 & Virgo \\
\hline 7 & 30 & Lib & 10 & 8 & 671 & Libra \\
\hline 8 & 31 & Sco & 10 & 0 & 689 & Scorpius \\
\hline 9 & 32 & Sgr & 14 & 0 & 699 & Sagittarius \\
\hline 10 & 33 & Cap & 28 & 0 & 713 & Capricornus \\
\hline 11 & 34 & Aqr & 41 & 0 & 741 & Aquarius \\
\hline 12 & 35 & Psc & 36 & 0 & 782 & Pisces \\
\hline 36 & 36 & Cet & 21 & 0 & 818 & Cetus \\
\hline 37 & 37 & Ori & 42 & 20 & 839 & Orion \\
\hline 38 & 38 & Eri & 10 & 9 & 901 & Eridanus \\
\hline 39 & 39 & Lep & 13 & 0 & 920 & Lepus \\
\hline 40 & 40 & $\mathrm{CMa}$ & 13 & 0 & 933 & Canis Maior \\
\hline 41 & 41 & $\mathrm{CMi}$ & 2 & 3 & 946 & Canis Minor, Procyon \\
\hline 42 & 42 & Arg & 3 & 8 & 951 & Argo Navis \\
\hline 43 & 43 & Hya & 19 & 5 & 962 & Hydra \\
\hline 44 & 44 & Crt & 3 & 5 & 986 & Crater \\
\hline 45 & 45 & Crv & 4 & 3 & 994 & Corvus \\
\hline \multirow{2}{*}{-} & 46 & Cen & 0 & 4 & 1001 & Centaurus \\
\hline & & & 777 & 227 & & \\
\hline
\end{tabular}

Notes. For each constellation the columns give the sequence number in Progym $\left(C_{B}\right)$ and Kepler $(C)$, the abbreviation we use, the number $N$ of stars in Progym, the number $N_{a}$ added in Kepler, the K-number of the first star in it (for quick reference of a K-number to its constellation), and the names as given in Kepler. Note that the modern constellations do not exactly match those used by Brahe; in particular the constellation Antinous has been subsumed in Aquila, and Argo is split since the 18th century in Carina, Pyxis, Puppis and Vela.

stars in his catalogue as follows: the first one In dextra tibia Ophiuchi (in the right shinbone of Ophiuchus) is identified with Oph 14 In dextra tibia and gives it its correct position; the remaining twelve are listed at the end of Ophiuchus, as Oph 26 to Oph 37.

- Kepler omits Gem 30 of Manuscript. (As one of the stars additional to Gemini in Manuscript, this star is also absent from Progym.) 
Table 2. Numbers $Z$ replacing the zodiacal signs in the machinereadable longitudes.

\begin{tabular}{rrrrrrrrrrrr}
\hline \hline 1 & $\Upsilon$ & 3 & I & 5 & $\Omega$ & 7 & $\bumpeq$ & 9 & $\chi^{\nearrow}$ & 11 & $\approx$ \\
\hline 2 & 8 & 4 & $\sigma$ & 6 & m & 8 & $\bar{m}$ & 10 & $\overline{6}$ & 12 & $\mathcal{- ( x}$ \\
\hline
\end{tabular}

- In Kepler Com 15 is identical to Com 2. Com 15 is not in Manuscript and Com 2 is not in Progym.

- Manuscript contains a star which would have been between Eri 13 and Eri 14 in Kepler, but Kepler omits it. Its position is almost the same as that of Eri 8, and it certainly refers to the same star. Progym lists only 10 stars for Eridanus.

If we do the counting, we see that Kepler adds three stars (the Novae and Com 15) and omits three stars (one each in Ophiuchus, Gemini, Eridanus) with respect to Manuscript, and thus ends up with the same total number of 1004 entries. With these exceptions, the order of the stars within each constellation is preserved between the various versions of the catalogue. Thus, UMa 13 is the same star in all three versions, and UMa 33 in Manuscript and Kepler is absent from Progym.

For each star a short description is followed by the ecliptic longitude and latitude, and the magnitude. The longitude is given in degrees $(G$ : integer), minutes $(M$ : integer or integer plus 0.5$)$, and zodiacal sign. The zodiacal sign is indicated with its symbol in the original catalogues, which we replace with an integer number $Z$ from 1 to 12 as shown in Table 2. The longitude in decimal degrees follows as

$\lambda=(Z-1) * 30+G+M / 60$.

The latitude is given in degrees ( $G$ : integer) and minutes ( $M$ : integer or integer plus 0.5$)$, plus a B or A indicating north (Borealis) or south (Australis). In the 1627 edition the B or A is often omitted, assumed implicitly to be the same as for the previous star. The latitude in decimal degrees follows as

$\beta= \pm(G+M / 60) ; \quad+/-$ for $\mathrm{B} / \mathrm{A}$.

The equinox of the coordinates is given in all three versions of the catalogue as 1600 (MDC) annum completum, or in modern terms AD 1601.00.

The last number given for each star is the magnitude. This is given as an integer, occasionally followed in Progym by a colon (:) or decimal point (.). According to Dreyer (1890, p. 354) these qualifications indicate somewhat brighter (:) and fainter (.), respectively. Manuscript and Kepler omit the magnitude qualifications. In lieu of a magnitude the original catalogues occasionally refer to a star as ne, for nebulous.

\section{Identification procedure}

To identify each star from the star catalogue of Brahe with a modern counterpart, we start with the selection from the Hipparcos Catalogue (ESA 1997) of all stars brighter than Johnson visual magnitude 6.0. We correct the equatorial Hipparcos star positions for proper motion, then precess the coordinates to equinox 1601.00 (old style, JD 2305824 ), and finally convert equatorial to ecliptic coordinates. For each entry in the old catalogue, we find the Hipparcos entry at the smallest angular distance.

\subsection{Conversion of the coordinates}

The Hipparcos coordinates are equatorial, for epoch 1991.25 (JD 2448349.0625 ) and equinox 2000.0. The proper motion thus has to be corrected for -390.25 years. We apply linear corrections separately to right ascension and declination, i.e. we ignore curvature in the proper motion. A major reason for using the Hipparcos Catalogue is that it provides errors on the proper motion, and that these errors are small. The typical accuracy of the proper motions given in the Hipparcos Catalogue is a few mas/yr, corresponding to less than an arcsec over the 390.25 period. The best measurements by Brahe have an accuracy of the order of an arcminute. The error in the Hipparcos position computed for 1601 contributes less than one percent to the error in the difference between the Brahe and Hipparcos positions if the error in the proper motion is less than 0.1 in $390.25 \mathrm{yrs}$, i.e. less than 0 ' $^{\prime} 015 / \mathrm{yr}$. This is the case for all but eight of the stars with $V<6.0$. The star with the largest error in proper motion among these eight is $\alpha^{2} \mathrm{Cen}$; at $\sigma_{\mu}=00^{\prime} 033 / \mathrm{yr}$, this error is less than 1 percent of the proper motion itself, and leads to a positional accuracy of $13^{\prime \prime}$ for epoch 1601 . Note that 3869 and 645 stars with $V<6.0$ have proper motions leading to displacements larger than $0{ }^{\prime} 1$ and $1^{\prime}$ over $390.25 \mathrm{yr}$, respectively. We conclude that correction for proper motion is important, but that the errors in this correction may be neglected.

For precession of the equatorial coordinates from 2000.0 to 1601.0 we use Eqs. (3.211) and (3.212-2) of Seidelman (1992).

Finally, for conversion of equatorial to ecliptic coordinates we use the value for the obliquity in 1601, computed with Eq. (3.222-1) of Seidelman (1992).

\subsection{Finding the nearest counterpart}

The angle $d$ between two stars with position $\lambda_{1}, \beta_{1}$ and $\lambda_{2}, \beta_{2}$ is computed from

$\cos d=\sin \beta_{1} \sin \beta_{2}+\cos \beta_{1} \cos \beta_{2} \cos \left(\lambda_{1}-\lambda_{2}\right)$.

For small angles $d$, computations based on this equation suffer from round-off errors. Therefore, if Eq. (1) results in $d<10^{\prime}$, we compute $d$ from

$d^{2}=\cos \beta_{1} \cos \beta_{2}\left(\lambda_{1}-\lambda_{2}\right)^{2}+\left(\beta_{1}-\beta_{2}\right)^{2}$.

\subsection{Deciding on the identification}

In many cases, the nearest Hipparcos match to the star in the Brahe catalogue, as found with Eqs. (1) or (2), is the obvious and unambiguous counterpart, as no other star is nearby. This may be seen most directly in the figures we provide in Sect. C, in which filled circles indicate a star from the Brahe catalogue and open circles stars from the Hipparcos Catalogue. Bigger symbols indicate brighter stars. A filled circle within an open circle is strongly indicative of a secure counterpart. Aries and Gemini are examples of constellations in which all stars are thus securely identified (Figs. C.30, C.31). In some cases the offset between the positions is large and indicates an error, but the identification is still secure with the nearest counterpart. Examples are $\beta$ Aur (near $+5,+5$ in Fig. C.17), and the three northern stars in Corona Borealis (Fig. C.8).

In a number of cases we consider not the nearest but another nearby Hipparcos match to the star in the Brahe catalogue as a secure identification. There are two possible reasons for this: first, the nearest Hipparcos star is much fainter than another unidentified Hipparcos star at a slightly larger angular 
distance; second, the nearest Hipparcos star is more plausibly identified with another star in Brahe's catalogue. An example of the first reason is $\mathrm{K} 101$, near -1 , +28 in Fig. C.4. K 101 is 0.7 from HIP 80309 ( $V=5.7$ ), and 10.5 from HIP 80331 ( $~ \eta$ Dra, $V=2.7$ ) and we consider HIP 80331 the secure counterpart. Because we consider a pair of Hipparcos stars to be one object when they are within $2^{\prime}$ from one another, most identifications of Brahe entries with a not-nearest Hipparcos star involve large positional errors. For example, K 646 lies about $61^{\prime}$ from the $6.0 \mathrm{mag}$ star HIP 65545 and about $78^{\prime}$ from the 3.4 mag star HIP 66249 (near $+4,+5$ in Fig. C.37). We consider HIP 66249 (= $\zeta$ Vir) a secure identification even if not the nearest Hipparcos star. An example of the second reason may be seen in the constellation Centaurus: Fig. C.58 shows convincingly that the position of the whole constellation is shifted to lower longitude in the Brahe catalogue. This implies that the counterpart of the middle star on the left is not HIP 67153 just south of it, but HIP 67669 almost a degree east of it. Another example of the second reason is $\kappa \mathrm{UMa}$ which forms a pair with $\iota$ UMa just north of it (near $-20,-7$ in Fig. C.2). $\kappa$ UMa is closest to HIP 44127 , but so is $\iota$ UMa, and $\kappa$ UMa may be securely identified with HIP 44471 just east of it. A further example is illustrated in Fig. C.5. K93 is closest to HIP 86219 and K 94 to the very close $\left(30^{\prime \prime}\right)$ pair HIP $86614+86620\left(=\psi^{1}\right.$ Dra $)$. From the brightness of the stars, however, we prefer to identify K 93 with HIP 86614+86620 and K 94 with HIP 87728. Dreyer (1916) agrees in the identification of K 93, but identifies K 94 with HIP 89937 ( $=\chi$ Dra), relying more on the description in Brahe's star catalogue of stars K 93-95 as a triangle. Rawlins (1993) corrects the position of K 94, and validates Dreyer's interpretation. All these examples illustrate the importance of identifying stars in context, rather than individually.

In a number of cases we cannot find a secure counterpart. Some of these are illustrated in Fig. C.3. K 68, K 72 and K 74 may be identified with nearby Hipparcos stars, which we consider probable rather than secure. K 75 is at an equal distance of two Hipparcos stars, and might be identified with either of them. The remaining stars cannot be identified with any Hipparcos star, either because no nearby matches are found, or - in the case of K67 - because the nearby star is identified with another star from the Brahe catalogue. Another constellation with stars we cannot confidently identify is Ophiuchus (the group near $+10,-16$ in Fig. C.19, see also Fig. C.20). We discuss uncertain identifications in Sect. B.

Finally, in three cases we find that two entries in the star catalogue are identical $(\mathrm{K} 10=\mathrm{K} 252 ; \mathrm{K} 339=\mathrm{M} 701$; $\mathrm{K} 483=\mathrm{K} 470$ ), and in other cases almost identical and probably referring to the same star. In two cases three entries appear to refer to the same star. These multiple entries are listed in Table 3.

We note that all three stars from Manuscript omitted in Kepler are in Table 3, i.e. they were presumably recognised by Kepler as double entries. We already noted that one of the stars added in Kepler, viz. K 483, is a repeat entry. Thus, if we accept that all 15 entries in the second column of Table 3 refer to the corresponding star in the first column, this implies that the 1004 entries in the Manuscript actually correspond to 990 stars; and that the 1004 entries in Kepler correspond to 992 stars. It may be argued that some entries from the second column of Table 3 refer to a hitherto unidentified star. This can be excluded in all cases where the angular separation between the catalogue entries is less than $2^{\prime}$, the resolution of the human eye. On the basis further of the absence of sufficiently bright candidate counterparts, as illustrated in the figures mentioned in
Table 3. Multiple entries in the Brahe catalogue.

\begin{tabular}{rrrrrrl}
\hline \hline & $\mathrm{K}$ & $\mathrm{K}(\mathrm{M})$ & $\mathrm{HIP}$ & $d_{1,2}\left(^{\prime}\right)$ & $\Delta M$ & See \\
\hline & 10 & 252 & 23734 & 0.0 & 0 & \\
$\mathrm{a}$ & 14 & 64 & 51808 & 13.8 & 0 & Fig. C.1 near $+6,+2$ \\
& 201 & 216 & 96441 & 7.8 & 0 & Fig. C.12 \\
& & 220 & 96441 & 1.0 & 2 & Fig. C.12 \\
& 333 & 360 & 80883 & 6.6 & 1 & Fig. C.19 near $-6,+6$ \\
& 336 & 347 & 86284 & 46.3 & 0 & Fig. C.21 \\
& & 364 & 86284 & 30.0 & 1 & Fig. C.21 \\
& 339 & M701 & 84012 & 0.0 & 0 & \\
$\mathrm{a}$ & 341 & 353 & 84893 & 22.3 & 0 & Fig. C.20 near $+8,-16$ \\
& 344 & 361 & 84880 & 11.2 & 1 & Fig. C.21 \\
& 345 & 362 & 86263 & 5.1 & 1 & Fig. C.21 \\
& 346 & 363 & 86565 & 16.5 & 1 & Fig. C.21 \\
& 470 & 483 & - & 0.0 & 0 & \\
& 584 & M94 & 39780 & 3.6 & 1 & \\
& 908 & M913 & 17593 & 0.4 & 0 & \\
b & 13 & 297 & 15520 & - & 0 & \\
b & 738 & 739 & 108036 & - & 0 & \\
b & $205-7$ & 219 & 102589 & - & 2 & \\
\hline
\end{tabular}

Notes. For each pair of K (or M) numbers the table lists the Hipparcos identification, with the difference in positions and magnitude of the Brahe entries and the approximate position in a figure in Sect. C. Effects of position corrections by Rawlins (1993, esp. footnote 77): a: no longer repeated entry, b: new repeated entry.

Table 3, we consider it most likely that all entries from the second column are in fact repeat entries.

While looking for identifications we occasionally encountered cases where an emendation to the Brahe star catalogue appears to be warranted. We describe these in Appendix A.

\subsection{Identifications by Dreyer and Rawlins}

Unavoidably, deciding between secure and probable identifications or between probable and possible identifications is sometimes subjective. We therefore compare our identifications with those by Dreyer (1916) and Rawlins (1993) in their editions of the Manuscript version of the star catalogue by Brahe, thus obtaining independent opinions.

To compare the identifications by Dreyer with those by us, we convert his identifications to a Hipparcos number. In most cases, Dreyer provides a Flamsteed number which is present in the Bright Star Catalogue (Hoffleit \& Warren 1991), allowing us to obtain the Hipparcos identification from the HR number via the HD number. In some cases a Flamsteed number given by Dreyer is not listed in the Bright Star Catalogue. For these we first check the SIMBAD data base: for any Flamsteed number in this data base the Hipparcos number is provided. In seven cases we have not found the Flamsteed number in the Bright Star Catalogue or in the SIMBAD database. For these (54 And, $51 \mathrm{Lib}, 31 \mathrm{Mon}, 2,4,9 \mathrm{Crt}$ and $35 \mathrm{Cam}$ ) we consulted the Flamsteed catalogue (Flamsteed 1725). To find the corresponding Hipparcos numbers we convert the positions of all Hipparcos entries brighter than $V=6.0$ to the equinox of the Flamsteed catalogue 1690.0 (old style) = JD 2338331 by first correcting for proper motion and then precessing the coordinates. We then find the Hipparcos entry with the closest coordinates to each of the seven Flamsteed stars. (In the constellation Camelopardalis some entries only provide a latitude, omitting the longitude; in counting entries to find number 35, we ignore these entries.) This leads to an unambiguous identification in all cases, with positional differences ranging from 0.3 to 1.0 . 
F. Verbunt and R. H. van Gent: Three editions of the star catalogue of Tycho Brahe

Table 4. First lines from the machine-readable tables KeplerE and Variants.

\begin{tabular}{|c|c|c|c|c|c|c|c|c|c|c|c|c|c|c|c|c|c|c|c|}
\hline M & B & K & $\mathrm{C}$ & $Z$ & $G$ & $M$ & $G$ & $M$ & & $V_{B}$ & HIP & $\mathrm{I}$ & $\mathrm{D}$ & $\mathrm{R}$ & V & $\Delta \lambda$ & $\Delta \beta$ & $\Delta$ & \\
\hline 336 & 307 & 1 & $1=\mathrm{UMi} 1$ & 3 & 23 & 02.5 & 66 & 02. & B & 2 & 11767 & 1 & 1 & 1 & 2.0 & -3.2 & 1.2 & 1.8 & \\
\hline 337 & 308 & 2 & $1=\mathrm{UMi} 2$ & 3 & 25 & 36. & 69 & 50.5 & B & 4 & 85822 & 1 & 1 & 1 & 4.3 & 1.6 & 3.4 & 3.5 & \\
\hline 338 & 309 & 3 & $1=\mathrm{UMi} 3$ & 4 & 03 & 24. & 73 & 50. & B & 4 & 82080 & 1 & 1 & 1 & 4.2 & 8.0 & 2.3 & 3.2 & \\
\hline 339 & 310 & 4 & $1=\mathrm{UMi} 4$ & 4 & 21 & 29. & 75 & 00 & B & 4 & 77055 & 1 & 1 & 1 & 4.3 & 15.2 & 4.5 & 6.0 & \\
\hline 340 & 311 & 5 & $1=\mathrm{UMi} 5$ & 4 & 24 & 52. & 77 & 38.5 & B & 5 & 79822 & 1 & 1 & 1 & 4.9 & -10.4 & 10.2 & 10.4 & \\
\hline $\begin{array}{c}\ldots \\
348\end{array}$ & 0 & 13 & $1=\mathrm{UMi} 13$ & 3 & 18 & 03. & 42 & 56. & B & 6 & 22783 & 3 & 1 & 4 & 4.3 & -158.1 & 26.0 & 118.2 & \\
\hline 349 & 0 & 14 & $1=$ UMi 14 & 4 & 21 & 38. & 57 & 55. & B & 6 & 51808 & 6 & 1 & 1 & 4.9 & -62.3 & 38.7 & 50.7 & \\
\hline 350 & 0 & 15 & $1=$ UMi 15 & 3 & 21 & 55. & 70 & 42. & B & 6 & 0 & 5 & 3 & 4 & 0.0 & 0.0 & 0.0 & 0.0 & \\
\hline 351 & 0 & 16 & $1=\mathrm{UMi} 16$ & 3 & 24 & 31. & 69 & 03. & B & 6 & 85699 & 1 & 1 & 4 & 5.8 & 61.4 & 27.8 & 35.2 & \\
\hline 344 & 0 & 9 & $1=\mathrm{UMi} 9$ & 4 & 27 & 20.5 & 70 & 18. & $\mathrm{~B}$ & 6 & 69112 & 1 & 1 & 1 & 4.8 & -10.5 & -0.8 & 3.6 & $\ldots \mathrm{M}$ \\
\hline 351 & 0 & 16 & $1=$ UMi 16 & 3 & 24 & 31. & 69 & 8. & B & 6 & 85699 & 1 & 1 & 1 & 5.8 & 61.4 & 22.8 & 31.4 & $\ldots \mathrm{M}$ \\
\hline $\begin{array}{c}\ldots \\
403\end{array}$ & 0 & 68 & $2=\mathrm{UMa} 48$ & 5 & 3 & 58. & 47 & 55. & B & 6 & 0 & 5 & 0 & 1 & 0.0 & 0.0 & 0.0 & 0.0 & K. . . \\
\hline 413 & 344 & 78 & $3=$ Dra 2 & 9 & 4 & 14.5 & 78 & 14.5 & B & 4. & 85819 & 1 & 1 & 1 & 4.9 & 22.7 & -3.2 & 5.6 & PM . \\
\hline $\begin{array}{c}\ldots \\
586\end{array}$ & 478 & 238 & $10=$ Cas 17 & 1 & 22 & 23.0 & 54 & 27.0 & B & 6 & 115990 & 1 & 3 & 1 & 4.9 & 15.9 & -24.3 & 26.0 & $\mathrm{~K}$. \\
\hline 586 & 478 & 238 & $10=$ Cas 17 & 2 & 22 & 33.0 & 54 & 27.0 & B & 6 & 7078 & 1 & 3 & 1 & 5.8 & -17.1 & -14.1 & 17.3 & . PM . \\
\hline 586 & 478 & 238 & $10=$ Cas 17 & 1 & 22 & 32.0 & 54 & 27.0 & B & 6 & 115990 & 1 & 3 & 1 & 4.9 & 6.9 & -24.3 & 24.6 & $\ldots \mathrm{C}$ \\
\hline
\end{tabular}

Notes. For explanation of the columns for the basic table KeplerE (above) and from the file with variants Variants see Sect. 4.

In seventeen cases Dreyer gives an identification consisting of the letter $\mathrm{P}$ followed by a roman and an arabic number. This refers to the catalogue of Piazzi (1803; we use the 1814 reprint). To find the corresponding Hipparcos numbers we convert the positions of all Hipparcos entries brighter than $V=6.0$ to the equinox of the Piazzi catalogue 1800.0 = JD 2378497 and find the nearest Hipparcos entry to each of the 17 Piazzi stars. This leads to an unambiguous identification in all 17 cases with positional differences ranging from $0{ }^{\prime} 06$ to $0{ }^{\prime} 19$.

In two instances Dreyer gives an identification consisting of a letter $\mathrm{G}$ followed by a number (and in both cases by a question mark). This refers to the catalogue by Groombridge (1838), which uses an equinox $1810.0=$ JD 2382149 . G3887 is 107' from HIP 112519 (corrected for proper motion and precession); however, G 3928 is only 0'03 from HIP 112519 . G 2807 has no Hipparcos counterpart with $V<6$, but HIP $90182(V=6.2)$ lies at 11.7 from it.

Some emendations to Dreyer's identifications that we consider probable are discussed in Sect. A.3.

To compare the identifications by Rawlins (1993) with those by us we convert his Bright Star Catalogue (HR) number for each of his identifications to an HD number using the Bright Star Catalogue (Hoffleit \& Warren 1991), and use the HD number to find the corresponding Hipparcos number.

\section{The machine-readable catalogues}

A large majority of the entries is identical in the three catalogues. To save unnecessary duplication we produce the basic machinereadable catalogue from Kepler to which we add the three stars from Manuscript that Kepler omits. This basic catalogue is referred to in the following as KeplerE. A second catalogue, referred to below as Variants, collects variants to the entries in KeplerE. A third catalogue, Names, contains the descriptions of the stars from Manuscript.

\subsection{The basic catalogue}

Kepler (1627) gives alternative positions for some stars, indicated with meus catalogus or with Piferus or abbreviated versions of these; these are omitted from KeplerE (and given in Variants). In KeplerE the emendations discussed in Sect. A.1 have been implemented.

The columns in KeplerE give the following information.

Columns $1-3$ give the sequence numbers of the stars in the three versions of the catalogue viz. $\mathrm{M}$ for the manuscript version (Brahe 1598), B for the printed edition of Brahe (1602), and K for the Kepler (1627) edition. KeplerE follows the sequence of entries in Kepler (1627); three stars from Manuscript omitted by Kepler (1627) are listed at the end of KeplerE. Thus KeplerE contains 1007 entries. To obtain the ordering of the stars/entries in the Manuscript (1598) or Brahe (1603) edition, one simply orders the table on Cols. 1 or 2, respectively. A sequence number zero indicates that the entry is absent in the corresponding catalogue.

Columns 4 and 5 indicate the constellation in which the star is listed in Kepler (1627). For convenience the constellation is indicated both with its sequence number C in Kepler (1627; see Table 1) and with the modern abbreviation (preceded by the equal sign =). We use Atn and Arg as abbreviations for Antinous and Argo, respectively, constellations which are no longer in use. Column 6 gives the sequence number of the star within the constellation in KeplerE.

Columns 7-14 copy the information of the original catalogue: Cols. 7-9 give the longitude ( $Z, G$ and $M)$, Cols. 10-12 the latitude ( $G, M$ and $\mathrm{A}$ or B), and Cols. 13, 14 the magnitude $V_{B}$ and its qualifier (blank, or :). The meaning of these numbers and qualifiers is explained in Sect. 2. Kepler (1627) often omits the B or A for the latitude, implicitly setting it equal to the value of the previous entry; KeplerE always gives B or A explicitly. A magnitude indicated as "ne" in the original catalogue is denoted 9 in KeplerE.

Columns 15-22 provide additional information from our analysis. Columns 15 and 16 give the number of the star in the Hipparcos Catalogue with which we identify the entry, and a flag I giving the quality of the identification, as explained in Sect. 3. The meanings of the identification flags are given in Table 5.

It should be noted that in general we limit our search for counterparts to Hipparcos stars with $V<6.0$, and the term 
Table 5. Meaning of flags I classifying our identifications.

\begin{tabular}{cl}
\hline \hline 1 & nearest star, secure identification \\
2 & not nearest star, secure identification \\
3 & probable identification, not secure \\
& because too far or too faint \\
4 & possible identification \\
& $\begin{array}{l}\text { other identification(s) also possible } \\
5\end{array}$ \\
6 & not identified \\
\hline
\end{tabular}

Table 6. Meaning of flags D and R for identifications by Dreyer (1916) and Rawlins (1993).

0 no identification given by Dreyer/Rawlins

1 identification by Dreyer/Rawlins identical to ours

2 identification by Dreyer/Rawlins identical to other of alternative identifications given by us

3 identification by Dreyer/Rawlins differs from ours

4 identification by Rawlins different due to corrected position

5 catalogue entry called "utter fake" by Rawlins

nearest in our identification flag ignores stars fainter than this limit. In the case of double entries it is not clear whether the star listed in the first or that in the second column of Table 3 should be considered the repeat entry. In general we have the entry closest to the Hipparcos star a flag 1 or 3, and the other doubles entries a flag 6 .

Columns 17 and 18 give flags $\mathrm{D}$ and $\mathrm{R}$ that compare our identification with those by Dreyer (1916) and Rawlins (1993), respectively. The meanings of these flags are given in Table 6.

Column 19 gives the visual (Johnson) magnitude $V$ of the Hipparcos object given in Col. 15. Columns 20, 21 give the differences in longitude $\Delta \lambda$ and latitude $\Delta \beta$ between the correct position (based on information from the Hipparcos Catalogue) and the catalogue entry in tabulated Minutes $M$. If the catalogue entry for minutes as computed from the position and proper motion in Hipparcos Catalogue is $M_{\mathrm{H}}$, and $M$ is the value actually given in the Brahe catalogue, then Cols. 20 and 21 give $M_{\mathrm{H}}-M$. Column 22 gives the difference $\Delta$ between correct and tabulated position in '.

\subsection{Catalogue with variants}

The second catalogue Variants collects five types of variants. First, it gives the original entries of Kepler (1627) for all entries which are emended in KeplerE to allow the reader to judge the validity of our emendations and if required to restore the original Kepler (1627) edition. Next, Variants gives entries from the 1598 manuscript version of the catalogue and from the 1602 edition when these are different from the corresponding entry in KeplerE. Finally we give the alternative positions given by Kepler (1627) as originating from meus catalogus or Piferus.

Each entry in Variants starts with the variant of an entry in KeplerE, and ends with a 4-character string indicating the origin of the variant. If the first character in this string is $\mathrm{K}$ the entry gives the original values of the Kepler (1627) edition (for which KeplerE gives emended values). If the second character is $\mathrm{P}$ the entry gives values from Brahe (1602) that differ from KeplerE. If the third character is $M$ the entry gives the values for the Manuscript version of Brahe (1598) that differ from KeplerE. This notation allows us to give variants which are common between different versions of the star catalogue in a single line.
Table 7. Frequency of flags D of Dreyer (1916) identifications as a function of the flags I of our identifications.

\begin{tabular}{lrrrrrrrrrr}
\hline \hline & \multicolumn{1}{c}{ KeplerE } & \multicolumn{1}{c}{ Progymnasmata } \\
$\mathrm{I} \backslash \mathrm{D}$ & 0 & 1 & 2 & 3 & all & 0 & 1 & 2 & 3 & all \\
\hline 1 & 11 & 896 & 0 & 22 & 929 & 2 & 735 & 0 & 15 & 752 \\
2 & 0 & 12 & 0 & 2 & 14 & 0 & 8 & 0 & 1 & 9 \\
3 & 11 & 5 & 1 & 1 & 18 & 1 & 2 & 0 & 1 & 4 \\
4 & 3 & 4 & 8 & 2 & 17 & 0 & 4 & 3 & 0 & 7 \\
5 & 11 & 0 & 0 & 3 & 14 & 3 & 0 & 0 & 2 & 5 \\
6 & 2 & 8 & 0 & 2 & 12 & 0 & 0 & 0 & 0 & 0 \\
all & 38 & 925 & 9 & 32 & 1004 & 6 & 749 & 3 & 19 & 777 \\
\hline
\end{tabular}

If the fourth character of the string is $\mathrm{C}$ or $\mathrm{P}$ the entry gives a variant given by Kepler (1627) with the characterization meus catalogus or Piferus, respectively.

Note that the identification procedure is done independently for all variant positions; when the variant position differs much from the KeplerE position, this may lead to a different identification.

\subsection{Catalogue with descriptions}

The third catalogue Names gives the descriptions of the stars as given in the manuscript version of the star catalogue (Brahe 1598). For convenience we add to this the sequence numbers $M$, $\mathrm{B}$ and $\mathrm{K}$ of each entry in the various editions of the catalogue.

\section{Analysis and discussion}

It is beyond the purpose of this paper to provide a full analysis of the star catalogue by Brahe and it different editions, but a few general remarks may be made.

\subsection{Identifications, emendations and the three versions}

In Table 7 , in the columns labelled all, we list the numbers of our identifications of the entries in KeplerE and in Progym. It is seen that only 14 entries remain unidentified in KeplerE, and 5 in Progym. A large majority of stars is securely identified.

The sixteen emendations that we apply to Kepler affect the number of identifications. In particular, eight lead to secure identifications (all with $\Delta \leq 3.2$ ) of previously unidentified stars. One other emendation leaves an unidentified star (K 67) unidentified, one gives a probable identification of a previously unidentified star (K 68), one improves the positional correspondence with its Hipparcos counterpart (K 801), and five lead to different identifications with better positions. Note that with one exception (K 801) all the emendations that we make to Kepler are taken from Progym and/or Manuscript. In Fig. 1 we show the change in position caused by our emendations to Kepler, and by different positions in Progym or Manuscript with respect to KeplerE. Not surprisingly, all emendations lead to better positions. Figure 1 shows that most differences between Progym and KeplerE are small, as are most differences between Manuscript and KeplerE. Remarkably, the figure also shows that in all cases where the positions differ strongly between Kepler and Progym and/or Manuscript, the positions in the older catalogues are better. 25 of the 26 entries from Progym that differ from the corresponding entries in KeplerE have the same identification in both versions, but K 547 is identified in KeplerE but unidentified in Progym. Similarly, 39 of 42 entries from Manuscript that differ from the corresponding entries in KeplerE have the same 
F. Verbunt and R. H. van Gent: Three editions of the star catalogue of Tycho Brahe

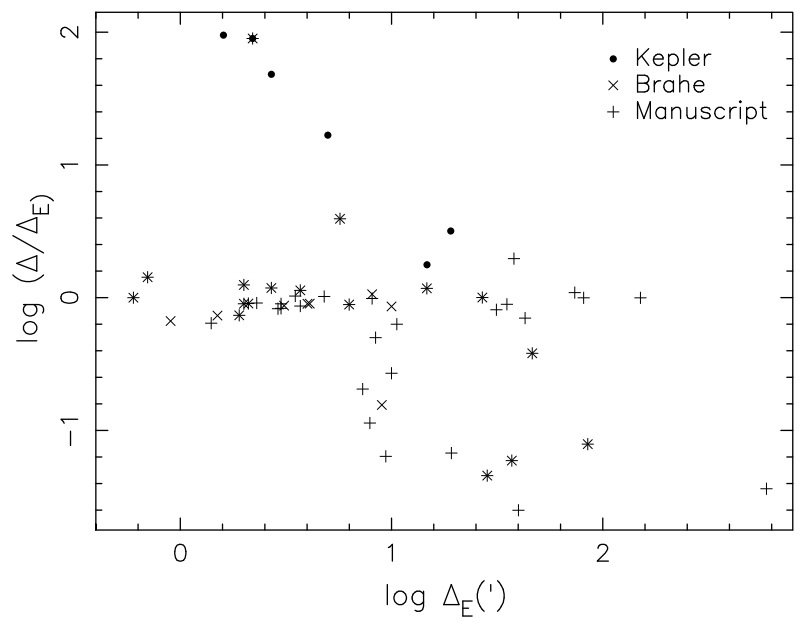

Fig. 1. Comparison of the accuracies of positions that are different in different versions of the star catalogue of Brahe. $\Delta_{\mathrm{E}}$ is the positional accuracy of KeplerE, $\Delta$ the positional accuracy of Kepler, Progym, or Manuscript. Note that symbols may be superposed when a variant position is common to several versions of the catalogue. Stars identified in one but not in the other catalogue are omitted.

identification in both versions, one (K251) is identified in KeplerE but not in Manuscript, and two (K 64 and K 300) have a different identification in KeplerE than in Manuscript.

\subsection{Comparison with Dreyer (1916) and Rawlins (1993)}

In Table 7 we compare the identifications as found by us with those given by Dreyer (1916). For both the Brahe (1602) and the emended Kepler (1627) versions, we find that our identifications agree with the earlier ones by Dreyer in most cases. We have identified a number of stars not identified by Dreyer, in some cases prefer another one from several plausible possibilities, and in some cases reject an identification by Dreyer.

The numbers in Table 7 should be read as indicative rather than exact, due to unavoidable arbitrariness in some classifications. The pair K $146 /$ K 147 is an example (see Sect. B): we flagged our identifications as secure $(I=1)$ and Dreyer's as wrong $(\mathrm{D}=3)$, but could have chosen ours as one of several possibilites $(I=4)$ and Dreyer's as an alternative to our choice ( $\mathrm{D}=2)$. Another example is the case of $\mathrm{K} 218$ in Cygnus. We have assigned HIP 106062 as its counterpart, because the closer and brighter counterpart has been assigned to K 440, a star in Pegasus. This is a reasonable choice if we assume that Kepler was aware of the proximity of K 440 to K 218 . If such was not the case, we could follow Dreyer and consider K 218 as a repeated entry for K 440, and our identification flag would be $\mathrm{I}=6$ rather than $\mathrm{I}=2$; and the flag for Dreyer's identification $\mathrm{D}=1$ rather than $\mathrm{D}=3$.

The sixteen emendations that we apply to Kepler also affect the numbers in Table 7.

Comparison of our identifications with those by Rawlins (1993) must be made with some care, because his identifications refer to an ideal version of the catalogue, that Brahe might have produced given the time, whereas our identifications refer to the catalogue in the versions edited by Brahe and Kepler. Thus Rawlins identifies the stars that Brahe actually observed, whereas we identify the stars closest to the catalogue positions.

In Table 8 we compare the identifications as found by us with those given by Rawlins for the emended Kepler edition. The three entries in KeplerE that do not occur in Manuscript
Table 8. Frequency of flags R of Rawlins (1993) identifications as a function of the flags I of our identifications for KeplerE.

\begin{tabular}{lrrrrrrr}
\hline \hline $\mathrm{I} \backslash \mathrm{R}$ & 0 & 1 & 2 & 3 & 4 & 5 & All \\
\hline 1 & 2 & 900 & 0 & 5 & 22 & 0 & 929 \\
2 & 0 & 11 & 0 & 0 & 3 & 0 & 14 \\
3 & 0 & 6 & 0 & 0 & 8 & 4 & 18 \\
4 & 0 & 9 & 1 & 0 & 7 & 0 & 17 \\
5 & 0 & 0 & 0 & 1 & 13 & 0 & 14 \\
6 & 1 & 11 & 0 & 0 & 0 & 0 & 12 \\
all & 3 & 937 & 1 & 6 & 53 & 4 & 1004 \\
\hline
\end{tabular}

are not discussed by Rawlins; all other entries are identified. In 937 cases our identification agrees with the one by Rawlins. We include in this four identifications (of K 583, K 718, K 120, and K 411) given by Rawlins (1993) that refer to one of a close pair of stars, whereas our identification refers to the other star of the pair. In each of these cases the pair is not separable with the naked eye, with a separation $<2^{\prime}$, and our identification refers to the star that is brighter in the Hipparcos Catalogue. In 911 cases the identifications given by Dreyer (1916), Rawlins (1993) and us all agree. In one case the identification given by Rawlins refers to one of two possibilities considered by us. In 53 cases Rawlins finds a different identification because he has corrected the catalogue position. For 4 entries, all in Ophiuchus, Rawlins concludes that Brahe invented positions without having observed them: they are "utter fakes" (see Fig. C.20).

This leaves 6 entries where our identification is different from that given by Rawlins. Five of these concern pairs of stars, with separations varying from 3 '.8 $^{\prime}$ to 10.5 , in which our suggested counterpart is closer to the catalogue position than the counterpart given by Rawlins. An example is shown in Fig. C.48. In four cases (K 183, K 209, K 671, K 870) our counterpart is the brighter star of the pair, in one case (K 804) only slightly fainter than the other star. In some cases, e.g. K 175, K 183 and K 209, Rawlins combines two stars separated by $8-11^{\prime}$ into one counterpart; in such cases we may choose the brighter and/or closer star as the counterpart, or leave the entry unidentified.

The corrections applied by Rawlins (1993) also affect the number of repeated entries, as indicated in Table 3.

\subsection{Accuracy}

Table 7 shows that 14 stars remain unidentified in our emended Kepler catalogue. Two entries in KeplerE have a secure identification, but no counterpart from the Hipparcos Catalogue: K 267 is SN 1572 and K 577 is Praesepe. To identify the fourteen unidentified stars would require that one accepts either a fainter counterpart, or a larger positional offset. It is necessary to note that such acceptance increases the probability of chance coincidences, i.e. of spurious identifications. That this is a serious problem may be concluded from the fact that we classified as "secure" four identifications of entries in Kepler (1627) that were identified with other counterparts after our emendation was applied. Thirteen of our unidentified entries are corrected by Rawlins (1993) to new positions, that allow him to identify them. One of our unidentified entries (K 175) is identified by Rawlins as the combined light of HIP $87045(V=6.47)$ and HIP 87119 $(V=6.83)$ two stars separated by 8.8 .

In Fig. 2 we compare the magnitude distributions of the stars in KeplerE to those of their securely identified counterparts. In our opinion, a difference between an Hipparcos magnitude and the magnitude assigned by Brahe cannot be called 

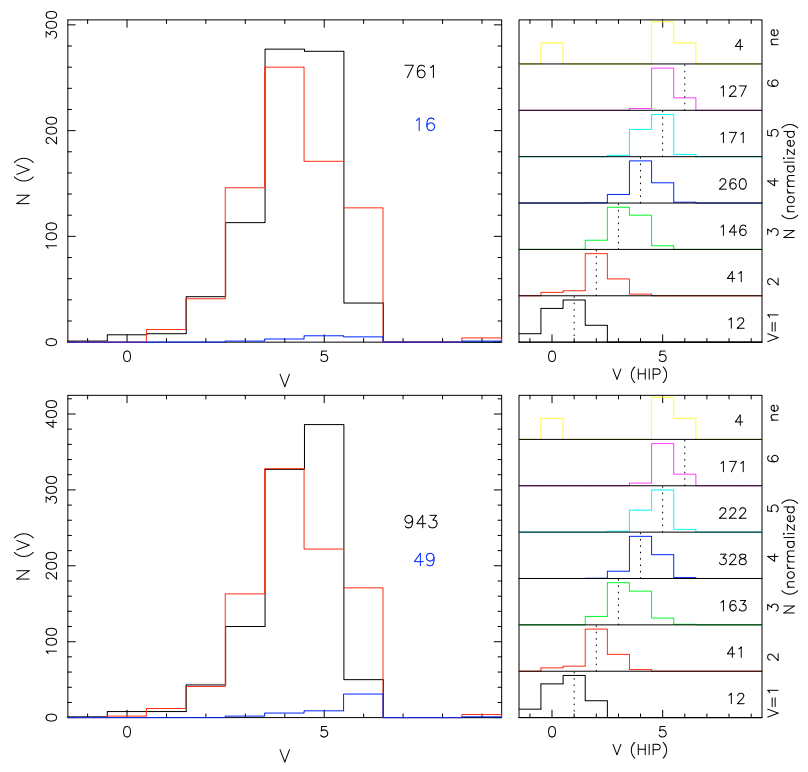

Fig. 2. Distribution of the magnitudes in Progym (above) and KeplerE (below). In the large frames the histograms indicate the magnitudes according to Brahe for stars which we have securely identified (red; flags $1-2$ in Table 5) or not securely identified (blue, flags 3-5), and the magnitudes from the Hipparcos catalogue for securely identified stars (black). The numbers of securely and not-securely identified stars are indicated. The small frames give the Hipparcos magnitude distributions for securely identified stars for each magnitude according to Brahe separately. The number of securely identified stars at each (Brahe) magnitude is indicated.

an error, since the magnitudes for Brahe correspond to a classification rather than a measurement. It is striking that Brahe's classification in general correlates well with the modern magnitude; only his magnitude 6 corresponds to mostly brighter stars in Hipparcos. The number of securely identified stars peaks at modern magnitudes 4 and 5, and rapidly drops for magnitude 6 . This lends support to our hesitance in accepting stars with modern magnitudes $V>6$ as feasible counterparts.

In Fig. 3 we show the offsets between the position in KeplerE and the position derived from the Hipparcos data, for longitudes and latitudes separately. We showed in Sect. 3.1 that errors in conversion of the modern data to the positions in 1601 are negligible, so that the offsets describe the errors in the position as given by Brahe (or Kepler). If the errors were fully random, we might expect their distributions to be Gaussian, but this is not the case: Gaussians that fit the peak of the distribution $\left(|\Delta \lambda|,|\Delta \beta|<5^{\prime}\right.$, say) have a width $\sigma \simeq 2^{\prime}$ and predict much smaller numbers at $|\Delta \lambda|,|\Delta \beta|>5^{\prime}$ than observed. The excess in the wings of the distributions with respect to a Gaussian description is presumably due to the correlations and buildup of errors when the position of a star is determined by measurement with respect to another star which already has a positional error. However, for large errors, $>10^{\prime}$ say, the possibility of a copying error must be considered, as illustrated by the differences between the different versions of the catalogue (see Fig. 1), and as proven for many cases by Rawlins (1993).

The average offsets $\Delta \lambda$ and $\Delta \beta$ are not zero, but for Gaussian fits to the central part of the distributions are both around $-0,5$. Together with the small but systematic dependence of the average offsets on longitude, also shown in Fig. 3, this suggests that a small part of the errors may be due to small errors in the position of the zero point in longitude and in the value of the obliquity that Brahe used. Brahe used an obliquity $\epsilon_{B}=23.525$
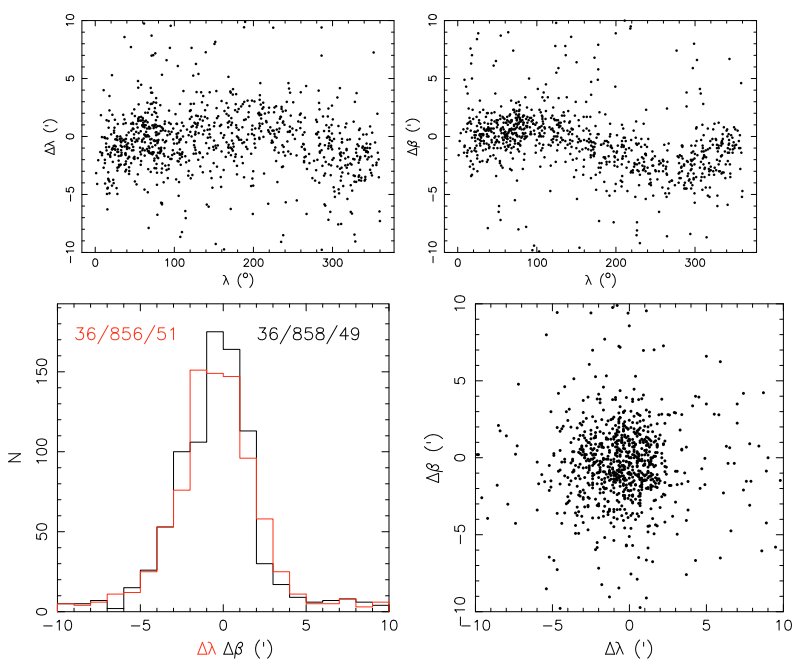

Fig. 3. Above: correlations of the differences in longitude $\Delta \lambda \equiv$ $\left(\lambda_{\mathrm{H}}-\lambda\right) \cos \beta$ and latitude $\Delta \beta \equiv \beta_{\mathrm{H}}-\beta$ of the entries in KeplerE and their secure Hipparcos counterparts (converted to 1601) with longitude. Below left: distributions of $\Delta \lambda$ and $\Delta \beta$. The numbers of sources with $\Delta \lambda, \Delta \beta<-10^{\prime}$, of sources included in the histogram $\left(-10^{\prime}<\Delta \lambda\right.$, $\Delta \beta<10^{\prime}$ ), and of sources with $\Delta \lambda, \Delta \beta>10^{\prime}$ are indicated. Below right: correlation between $\Delta \lambda$ and $\Delta \beta$.

(Brahe 1602, p. 18 and p. 208) whereas the correct value for 1601 according to modern determinations (see Sect. 3.1) was $\epsilon=23: 491$. For small declinations $\delta$, the resulting error in latitude $\Delta \beta \equiv \beta-\beta_{B}$ due to the error $\Delta \epsilon \equiv \epsilon-\epsilon_{B}$ after converting equatorial to ecliptic coordinates may be written

$\cos \beta \Delta \beta \simeq-\sin \alpha \cos \epsilon \Delta \epsilon=1.9 \sin \alpha$.

This dependence on right ascension is clearly seen in Brahe's data, as illustrated in Fig. 4.

Brahe also assumed that refraction at altitudes above $20^{\circ}$ is negligible, whereas a modern estimate would give 0.7 at the altitude of the equatorial pole for Hven (latitude almost $56^{\circ}$; the actual refraction depends somewhat on weather circumstances). Declination measurements with respect to this pole would thus all be offset by -0.7 , and this would lead to a systematic offset in $\beta$. Even though the real situation would be more complicated, involving differences in refraction errors between stars measured at different altitudes (see Fig. 4), we think that this offset largely explains the overall offset in $\beta$ seen in Figs. 3 and 4, which averages -0.5 , and is already present in the positions of Brahe's nine standard stars (Fig. 4; see also Dreyer 1890, p. 387).

If $\Delta \lambda$ and $\Delta \beta$ were distributed as Gaussians centered on zero, the distribution of the total position errors $\Delta \equiv \sqrt{\Delta \lambda^{2}+\Delta \beta^{2}}$ could be written

$$
\begin{aligned}
N f(\Delta \lambda) f(\Delta \beta) d \Delta \lambda d \Delta \beta & =\frac{N}{2 \pi \sigma^{2}} \mathrm{e}^{-0.5 \Delta \lambda^{2} / \sigma^{2}} \mathrm{e}^{-0.5 \Delta \beta^{2} / \sigma^{2}} d \Delta \lambda d \Delta \beta \\
& \equiv N f(\Delta) d \Delta=N \frac{\Delta}{\sigma} \mathrm{e}^{-0.5(\Delta / \sigma)^{2}} d \frac{\Delta}{\sigma}
\end{aligned}
$$

The maximum of this function is at $\Delta=\sigma$. In Fig. 5 we show the distribution of $\Delta$. The observed error distribution is similar to Eq. (4), but has more measurements at large $\Delta$ than expected from Eq. (4) with $\sigma=2$ because the observed distributions of $\Delta \lambda$ and $\Delta \beta$ are not centered on zero (a small effect) and show excesses at higher offsets with respect to a Gaussian (the dominant effect).

Using Kolmogorow-Smirnov tests to compute the probability that the distributions of the errors $\Delta$ in the range $\Delta<10^{\prime}$ for 
F. Verbunt and R. H. van Gent: Three editions of the star catalogue of Tycho Brahe
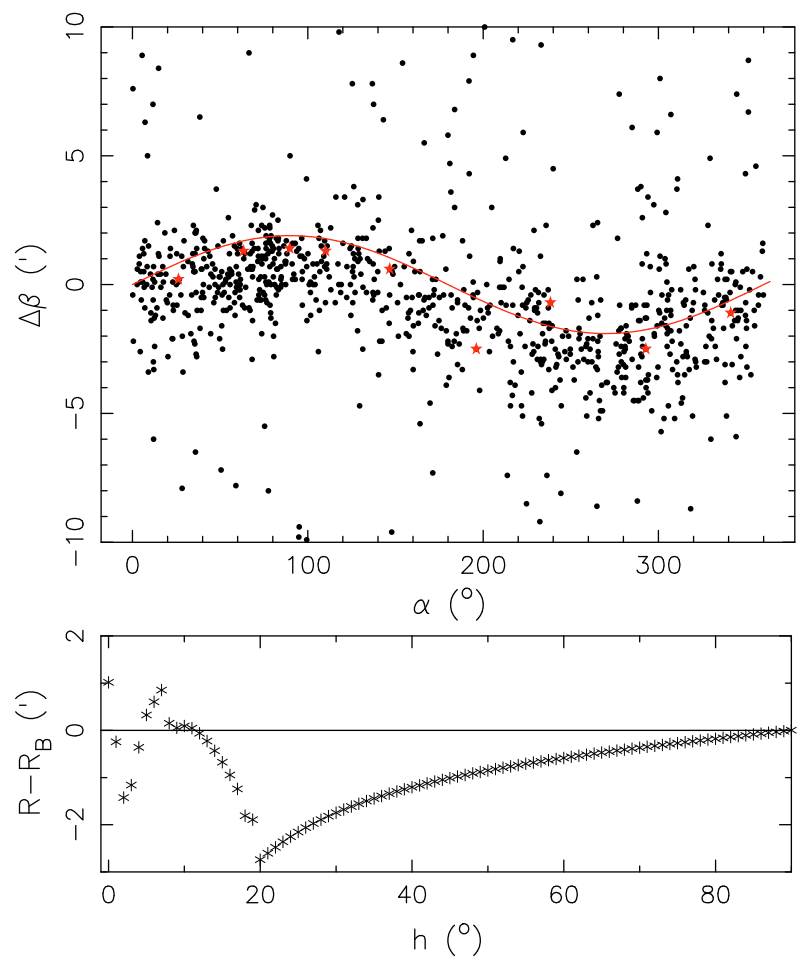

Fig. 4. Above: latitude errors $\Delta \beta$ in KeplerE as a function of right ascension. The nine standard stars of Brahe are indicated with a red star; the red line indicates 1:9 $\sin \alpha$ (see Eq. (3)). Below: the difference between modern values for diffraction and the values used by Brahe (zero at $h>20^{\circ}$ ) for stars, as a function of altitude $h$ (Saemundsson 1986; Meeus 1998; Brahe 1602, p. 287).

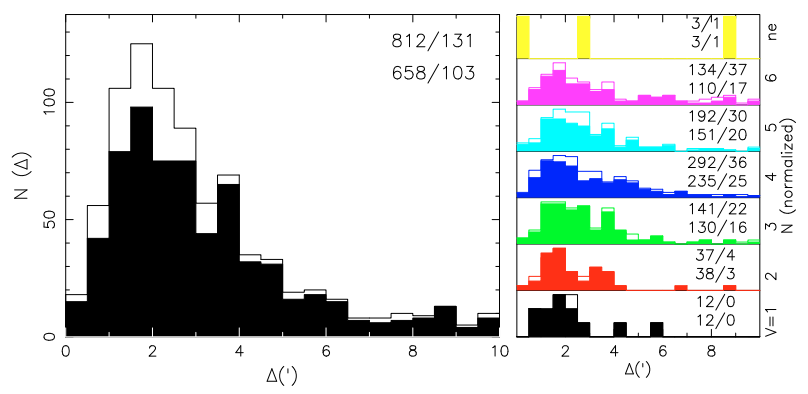

Fig. 5. Distribution of the position errors $\Delta$ in Progym (solid histograms) and in KeplerE (open histograms) for all securely identified stars (left) and for the securely identified stars at each Brahe magnitude separately (right). The numbers indicate the number of stars included in the plot (i.e. with $\left.\Delta<10^{\prime}\right)$ and those excluded $\left(\Delta \geq 10^{\prime}\right)$.

$V=n$ is the same as for $V=n+1$ we find no significant differences. However, the distribution of $\Delta$ for $V \leq 2$ is significantly different from that for $3 \leq V \leq 5$ (probability for being identical is 0.003). Comparing the differences between the distributions of $\Delta$ over the full range of $\Delta$, i.e. including large offsets, we find a significant difference between $V=5$ and $V=6$ (probability for being identical is 0.001 ). We conclude that the positions of stars with Brahe magnitudes 1 and 2 are better than those of fainter stars; and that a larger fraction of the positions of stars with magnitude 6 is wrong by more than $10^{\prime}$ than for positions of fainter stars.

Acknowledgements. We thank Dr. Gertie Geertsema for help in photographing Kepler. This research has made use of the SIMBAD database, operated at CDS, Strasbourg, France, and was supported by The Netherlands Organisation for Scientific Research under grant 614.000.425.

\section{References}

Baily, F. 1843, MNRAS, 5, 279

Brahe, T. 1598, Stellarum octavi orbis inerrantium accurata restitutio (manuscript), ed. A. Dreyer, 1916, 333

Brahe, T. 1602, Astronomiae Instauratae Progymnasmata, ed. Dreyer, 1915, 258

Dreyer, J. L. E. 1890, Tycho Brahe. A picture of scientific life and work in the sixteenth century (Edinburgh: Adam and Charles Black)

Dreyer, J. L. E. 1915, Tychonis Brahe Dani Scripta Astronomica, Vol. II (Kopenhagen: Gyldendal)

Dreyer, J. L. E. 1916, Tychonis Brahe Dani Opera Omnia, Vol. III (Kopenhagen: Gyldendal)

ESA 1997, The Hipparcos and Tycho Catalogues (Noordwijk: ESA Publications Division), SP-1200

Flamsteed, J. 1725, Historia Coelestis Brittanicae (London: H. Meere), 3

Groombridge, S. 1838, A catalogue of circumpolar stars, ed. G. B. Airy (London: Murray)

Hoffleit, D., \& Warren, W. H. 1991, Bright Star Catalogue, 5th Revised Ed., Astronomical Data Center, NSSDC/ADC, http://cdsarc.u-strasbg. $\mathrm{fr} / \mathrm{viz}$-bin/Cat?V/50

Kepler, J. 1627, Tabulae Rudolphinae (Ulm: Jonas Saur)

Meeus, J. 1998, Astronomical Algorithms ${ }^{2}$ (Richmond: Willmann-Bell)

Piazzi, G. 1803, Praecipuarum stellarum inerrantium positiones mediae (Palermo: Typis Regiis), reprinted 1814

Rawlins, D. 1993, DIO, 3, 1

Saemundsson, T. 1986, Sky \& Telescope, July, 72, 70

Seidelman, P. K. 1992, Explanatory supplement to the astronomical almanac (Sausalito, California: University Science Books)

Wesley, W. G. 1978, JHA ix, 42

\section{Appendix A: Emendations}

We have emended some obvious errors in Kepler (1627). Positional errors are obvious if they place a star well outside the constellation; in general we emend them only if the correct version appears in the Brahe (1603) version. In the following, we abbreviate Meus Cat for an alternative position labelled Meus Catalogus in Kepler (1627). In this section reference to Dreyer is short for Dreyer (1916) and reference to Rawlins for Rawlins (1993).

\section{A.1. Applied emendations}

We list errors in Kepler (1627) that we emended.

$\mathrm{K} 67,68$ have $Z=\sigma$ and $\Omega$ for their longitudes in Kepler, and $\Omega$ and $\mathrm{m}$, respectively, in Manuscript. Because the description Tertia (the third) for K 69 couples it to that of K 68 Secunda earundem (the second of the same [sc. stars]) we apply the correction. Whether or not we apply the emendation, we don't find close counterparts for these stars.

K 145 has $\Omega$ in Kepler, but $M$ in Manuscript. Because K 145 is described as following K 144 (hanc sequens), we follow Manuscript.

K 238 has $Z=\Upsilon$ in Kepler for the longitude, but $\succ$ in Progym and Manuscript. Because the star is described together with K 237 and K 239 as in the bench (scabellum) of the throne on which Cassiopeia sits, it should be near these two stars.

K 378 has $29^{\circ}$ in the latitude in Kepler, too far from the other stars of Sagitta. We emend to the value $39^{\circ}$ of Progym and Manuscript.

K 499, 500 have southern latitude A in Kepler, but northern latitude B in Progym and Manuscript.

K530 has southern latitude A in Kepler, both Manuscript and Progym give B.

Kepler gives $Z$ of K 539 as $\succ$; both Manuscript and Progym give II.

The magnitudes of K 535-545 are erroneously replaced in Kepler with those of K 536-546, and the magnitude of K 546 
is left empty. This is due to an erroneous shift by one line of the magnitude column, starting with a meus cat alternative position for K 535.

K 547. Manuscript has B for the latitude of K 547, whereas Progym and Kepler give A. B is clearly correct, as it fits the description of the star Quae est inter binas praec. in $\square$ colli (which is between the two leading stars in the square at the neck) and lies within 0.3 of HIP 19171.

K 685, 686 are A(ustralis) in Kepler but B(orealis) in Manuscript. The southern positions give no near counterparts, whereas the northern positions do. The descriptions for K 685 Superior et Orientalior (North and East, sc. of K 684) and K 686 Quae hanc sequitur (Which follows this one) imply positions north of K 685, and thus B.

K 801. Manuscript, Progym and Kepler all have B for the latitude of this star, close to K 802, and with no near counterpart. A latitude A gives an excellent match both to the otherwise unidentified o Psc, and to the description In lineo boreo a connexu praecedens (in the northern line leading the connection).

K 934. Manuscript and Progym have $G=11$ in the longitude, Kepler has $G=19$. The descripton Quae in fronte ad dextram aurem (Which is in the forehead at the right ear) is not compatible with the position in Kepler.

K 972, K 973. Manuscript and Progym have $\Omega$ for longitude, Kepler has m.

\section{A.2. Not-applied emendations}

We list errors in Kepler (1627) which we decided not to emend.

K 59-61 all three have magnitude 5 in Manuscript, and 3 in Kepler. Their counterparts indicate that the Manuscript magnitudes are correct.

K 534 has the same position in Manuscript, Progym, and Meus Cat which differs from that in Kepler. The Manuscript position give a better match to Electra (see Fig. C.34).

K 581. Manuscript has B for the latitude of K 581, whereas Progym and Kepler give A. The manuscript position gives a better match with HIP $42911(=\delta \mathrm{Cnc})$ at $0{ }^{\prime} .9$.

\section{A.3. Emendations to Dreyer}

In a number of cases the Flamsteed number is accompanied by an (extended) Bayer identification that is different from the one given in the 5th edition of the Bright Star Catalogue (Hoffleit \& Warren 1991). For example 35 Lib (K $679=$ HIP 76126) is listed as $\zeta^{1}$ by Dreyer and as $\zeta^{4}$ Lib in the Bright Star Catalogue. Similarly with $\pi$ Ori (K 860-865). Since we use only Flamsteed numbers to identify the counterpart we do not emend such differences.

K 580 Dreyer gives Flamsteed No. 41 for $\gamma$ Cnc; correct is 43.

K 646. Dreyer gives Flamsteed No. 70 for $\zeta$ Vir; correct is 79.

K 990. Dreyer gives Flamsteed No. 23 for $\theta$ Crt. Correct is 21 .

K 263 and K 264. Dreyer gives no identification for K 263 and P.IX.37 = HIP 47193 for K 264. From the position HIP 47193 is a better match for K 263, which when accepted leaves K 264 unidentified by Dreyer.

\section{Appendix B: Notes on individual identifications}

In this section reference to Dreyer is short for Dreyer (1916) and reference to Rawlins for Rawlins (1993).

K 10-13 lie in a straight line in Fig. C.1 (lower right corner), without close counterparts. All four identifications we choose lie to the northwest. K 12 lies rather closer to HIP 24914, 63'.4 to the southeast, and this star is an alternative identification. The counterpart suggested for K 12 by Dreyer, HIP 24348 is too faint and still not very close $(V=6.5, \Delta=45.3)$.

$\mathrm{K} 14$, near $+5.5,+1.5$ in Fig. C. 1 , is very close $\left(14^{\prime}\right)$ to $\mathrm{K} 64$ in Ursa Maior, and refers to the same star.

$\mathrm{K} 15$, near $-6,+15$ in Fig. C.1, is tentatively identified by Dreyer with Groombridge 2708, possibly identical to HIP 90182 at 11.7 from the Groombridge position. We consider HIP 90182 too faint, at $V=6.2$.

$\mathrm{K} 18$, near $-12,+14$ in Fig. C.1, is tentatively identified by Dreyer with Groombridge 3887; however, HIP 112519, our counterpart for K 18, corresponds to Groombridge 3928.

$\mathrm{K} 26$, near $-16,+16$ in Fig. C.2, has no near counterpart. It lies between two faint stars HIP $50685\left(V=5.9, \Delta=111^{\prime}\right.$ East $)$ and HIP 47594 ( $V=5.7, \Delta=102^{\prime}$ West), but we prefer the brighter star slightly further West. Dreyer agrees.

$\mathrm{K} 29$, near $-15,+10$ in Fig. C.2, is not identified by us. We consider HIP 47911, the counterpart given by Dreyer, too faint $(V=6.6)$ and far $(\Delta=46.7)$ to be viable.

K 93,94. We identify K 93 with the close pair HIP 86614/86620 $\left(=\psi^{1}\right.$ Dra) and K 94 with HIP 87728 . For K 94 Dreyer prefers HIP 89937 ( $=\chi$ Dra) as counterpart. See Fig. C.5

$\mathrm{K} 101$, near $-1,+28$ in Fig. C.4, lies very close to HIP 80309 $\left(V=5.7, \Delta=0{ }^{\prime} 7\right.$ ), but its much brighter neighbour is our preferred counterpart.

$\mathrm{K} 146$ and $\mathrm{K} 147$, near $-0.5,+17.5$ in Fig. C.7. Our identification for K 146 is that of K 147 in Dreyer, and vice versa, because we identify with the nearest object, whereas Dreyer follows order in longitude.

$\mathrm{K} 175$, near $+7,+18$ in Fig. C.9, is listed in the catalogue as nebulous, and is identified by Dreyer with HIP 87280, a Be star. Although variable, HIP 87280 is too faint $(V=6.8)$ and too far $\left(\Delta=50^{\prime}\right)$ to be a viable counterpart. Rawlins identifies K 175 with the combined light of HIP $87045(V=6.47)$ and HIP $87119(V=6.83)$ two stars separated by 8.8 . We leave K 175 unidentified.

K 183, near $-16,+6$ in Fig. C.9, is identified by Dreyer with HIP 86534, but this star is at a distance $\Delta=77^{\prime}$, whereas our identification, which corresponds to $53 \mathrm{Boo}$, lies at 1.2 from K 183. Rawlins identifies this entry with the combined light of 52 Boo (=HIP 75973, $V=5.0$ ) and 53 Boo, separated by 10.5.

K 191, near $-2.5,-3$ in Fig. C.10, is identified by Dreyer with HIP $92398\left(=v^{1}\right.$ Lyr $)$, which is both fainter and further $(V=5.9$, $\left.\Delta=14^{\prime}\right)$ as our identification HIP $92405\left(=v^{2}\right.$ Lyr $)$.

$\mathrm{K} 209$, near $+4.5,+14$ in Fig. C.11, is identified by Dreyer with HIP 99639, both fainter and further $(V=4.8, \Delta=9$ '.5) than our suggested counterpart HIP 99675. Rawlins identifies this entry with the combined light of these two stars.

$\mathrm{K} 216$ is at 7.8 from $\mathrm{K} 201 ; \mathrm{K} 220$ is at 1.0 from $\mathrm{K} 201$ (Fig. C.12). We think that these three entries refer to the same star, HIP 96441. Dreyer identifies K 201 as we do, but identifies 
K 216 and K 220 both with HIP 96895 , but this star is $54^{\prime}$ from K 220.

$\mathrm{K} 218$, near $+13,-12$ in Fig. C.11. HIP 106140, the counterpart suggested by Dreyer, is brighter and closer than our suggested counterpart, but it is already taken by $\mathrm{K} 440$.

\section{K 220: see K 216.}

K 237-239. Notwithstanding implied position errors of 224', $161^{\prime}, 68^{\prime}$, it is tempting to agree with Hevelius, Dreyer and Rawlins and identify these three stars with HIP 9598, HIP 9480 and HIP 9009, respectively, since these counterparts are not matched by other entries in Brahe's catalogue, even though rather brighter than the counterparts we choose because of their positional matches, HIP 5926, HIP 7078 and HIP 7965. See Figs. C.13, C.14. Rawlins indeed corrects the positions. Note that the Brahe catalogue give magnitudes 6 to K 237-239. K 249 lies very close to K 300 (Fig. C.14), but we do not consider this to be a double entry: see K 297-300.

K 254, near +20, -9 in Fig. C.13, lies between HIP 33449 and HIP 32489. We choose the HIP 33449 as a counterpart as it is brighter; Dreyer chooses HIP 32489, which is fainter but closer $(V=5.3, \Delta=55.3)$

$\mathrm{K} 264,265$, near $+14,+18.5$ in Fig. C.13, form a pair close to the pair HIP 51384 and HIP 51502. If we follow Dreyer and identify K 265 with HIP 51502 (at 6'0), K 264 is without counterpart. We have chosen to identify the pair, accepting the larger offsets. Neither solution is very satisfactory.

$\mathrm{K} 267$ is SN 1572. The positional error is rather large, about 15', mainly in longitude.

K 294. Rawlins makes a large correction to its position and identifies it with HIP 19949 ( $V=5.2)$.

K 297-300 are the four additional stars (indicated blue) top left in Fig. C.15. K 297 and K 300 are close to HIP $15520(\Delta=$ 2 '.8) and HIP $14862(\Delta=16.3)$, respectively, whereas K 298, 299 have no nearby counterparts. This is the choice made by Dreyer. Looking at the figure we have the distinct impression that the correct position of this group of four stars is about $10^{\circ}$ (mainly towards the south) from the catalogued positions. We give our identifications accordingly. Rawlins identifies K 297 with HIP 15520 and corrects the positions of K 298-300 to identify K 298 with HIP 16281, K 299 with HIP 16292 and K 300 with HIP 16228.

$\mathrm{K} 317$. The Kepler position is closest to the counterpart of K 316, so that we choose HIP 27673 as counterpart (near $+3,-1$ in Fig. C.17, the two entries of the Brahe catalogue - in blue are merged). For the position of Meus Cat HIP 27673 is in fact the star closest to $\mathrm{K} 317$.

$\mathrm{K} 319$, near $-2,-5$ in Fig. C.17, lies between HIP 25471 ( $V=$ 5.9, $\Delta=48$ '. 1 ) and our preferred counterpart HIP 24879, brighter and at the same distance $(V=5.1, \Delta=48.5)$. Dreyer takes HIP 25541 as counterpart $\left(V=5.1, \Delta=58^{\prime}\right)$.

K 336, K 347 and K 364 have virtually the same position, and all correspond to HIP 86284 ( $=\mu$ Oph). See Fig. C.21.

K 341: see K 353.

K 344-347. We agree wih Dreyer in considering K 361 to K 364 as repeat entries for K 344 to K 347 . Alternatively, one may identify the pair K345/K 362 with the pair HIP 86263/HIP 86266. See Fig. C.21.

K 347: see K 336.

K 353-364. Kepler annotates these entries with: Desunt in meo seqq. ad finem. Vide Classem secund. (The following until the end are absent in mine, see Second Class). Kepler refers to the stars in the catalogue of Ptolemaios that Brahe omitted in his catalogue as the Second Class, and lists these separately, on pages following his edition of the Brahe catalogue.

K 353-357 are illustrated in Fig. C.20. Our interpretation has K 353 as a repeat entry for K 341 ; and identifies K 354 with HIP 85207 and K355-357 with HIP 84970, HIP 85340 and HIP 86755, respectively. An alternative possiblity would be to identify K 341 with HIP 85207 and K 354 with HIP 84626, but we consider this less likely. Dreyer gives no identifications for K 353-357. Rawlins argues that K 354-357 are utter fakes.

K 360-364 are all repeat entries (see Table 3) K360 is near -6 , +6 in Fig. C.19. For K 361-364 see Fig. C.21. One may consider the pair K $362-\mathrm{K} 345$ to correspond to the pair HIP 86266-HIP 86263, but we consider this less likely, because the orientation is not right and because the two entries preceding K 362 and the two following are all repeat entries.

$\mathrm{K} 470$ is a rather confusing entry. It is repeated exactly as K 483 , apparently unnoticed by Kepler; Manuscript gives it at the location of K 470 only, and Progym gives it at the location of K 483 only. It has no nearby identification. Kepler annotates K 470 Meus solus. Forte eadem (Mine only. Accidentally in the same place). This may refer to its absence at this location in Progym and suggests that Kepler thought it as almost at the same coordinates as K 469. Did he consider 28 for $M$ in longitude as an error for 18 ?

K471-473. From the positions in the catalogue, K471 and K 472 both lie closest to HIP 60697 (near -4.2, -0.7 in Fig. C.28) and K 473 is close to HIP 60746, leaving HIP 60904 (near $-3.2,-1.6$ ) without catalogue counterpart. The catalogue describes K 471-473 as following one another, hence we prefer to identify K 472 with HIP 60746 and K 473 with HIP 60904. Dreyer agrees.

$\mathrm{K} 484=$ HIP 8832 corresponds to HR 545+546 $\left(\gamma^{1}+\gamma^{2}\right.$ Ari $)$.

K 517 is identified with the northern of the pair $\theta^{1,2}$ (HIP 20885 and HIP 20894) by Dreyer, but actually is closer to the southern star. See Fig. C.33.

K 534 is Electra in the Pleiades, as shown by its position in Manuscript (see Fig. C.34).

K 543 lies between HIP 21683 and HIP 21673, somewhat closer to the latter (Fig. C.33). We list the brighter star as the counterpart. HIP 21673 is fainter $(V=5.1)$ but closer $(2.4)$, and is listed by Dreyer as the counterpart.

K 547. The position in Manuscript gives excellent agreement with HIP 19171 (at 0.3), which is the identification also given by Dreyer. The position given in Progym and Kepler does not lead to a good identification. See Fig. C.32.

K 556, near $+5.5,+5.5$ in Fig. C.31, lies between HIP 36393 (at 8.9) and HIP 36492. Dreyer gives HIP 36393 as identification, we choose the closer star.

K 577 is the open star cluster Praesepe; Dreyer identifies it with the brightest star (at $V=6.3$ ) in Praesepe, $\epsilon \mathrm{Cnc}=\mathrm{HIP} 42556$

$\mathrm{K} 607$, near $-7,-3$ in Fig. C.36, is identified with HIP 51585 (46 Leo, near $-0.5,-0.5$ ) by Dreyer; too far to be believable. HIP 50333, near $-4,-2$, is closer.

$\mathrm{K} 625$, near $-3.0,+12$ in Fig. C.36, is identified with 7.3 mag star HIP 52240 (39 LMi) by Dreyer, too faint to be believable. 
We think our identification with HIP 52422 is secure, as this is the only sufficiently bright star near K 625 .

K 646, near $+4,+5$ in Fig. C.37, is securely identified with HIP 66249 ( $\zeta$ Vir) even though the rather fainter HIP 65545 is somewhat closer at 61.2.

$\mathrm{K} 650$, near $+13.5,-1.0$ in Fig. C.37, is possibly identified with HIP 68940, as no other sufficiently bright star is nearer. Dreyer gives no identification.

K 676, near $-2,+1$ in Fig. C.38. We accept a counterpart with $V=6.1$ as no brighter stars are nearby; the identification by Dreyer, HIP 75294, is fainter and further $\left(V=6.5, d=33^{\prime}\right)$.

$\mathrm{K} 687,688$, near $-2.5,-9$ and $-2.5,-3.5$ in Fig. C.38, have better positions in Manuscript, i.e. closer to the counterparts, than in Kepler.

K 713 is closer to $\alpha^{2}$, the brighter and southern star of the pair $\alpha^{1}, \alpha^{2}$ Cap (Fig. C.42). Dreyer gives $\alpha^{1}$ as identification.

K 717, 718 and 720 are listed "ne", but are well identified with stars (see Fig. C.41).

$\mathrm{K} 737$, near $+9.5,+3$ in Fig. C.41, is rather far from its identification, but no star with $V<6$ is closer.

$\mathrm{K} 738$, near $+11.5,+0.5$ in Fig. C.41, is identified (by us and Dreyer) with HIP 108036, which leaves K 739, right next to it, without counterpart, unless we are willing to accept a $V=$ 6.3 star.

$\mathrm{K} 759$, near $+4,-3$ in Fig. C.43) is tentatively identified by us with HIP 112542 (near $+7,-3$ ) and by Dreyer with HIP 110778 (near $+1,-4)$.

$\mathrm{K} 772$, near $+17,-12$ in Fig. C.43). Our identication is both brighter and closer than the identification given by Dreyer $\operatorname{HIP} 116889(\Delta=14.2)$.

$\mathrm{K} 804$, near $+17,+3$ in Fig. C.44). Our identification is closer than, but slightly fainter than the identification given by Dreyer and Rawlins, $\rho$ Psc $=\operatorname{HIP} 6706\left(\Delta=5^{\prime}\right.$.6 $)$.

$\mathrm{K} 808$, near $+13,+12$ in Fig. C.44). Our counterpart has $V=6.1$. K 870 (Fig. C.48) is identified with HIP 26268 by Dreyer $(V=$ 5.2, $\Delta=5$ 5.1). Our identification HIP 26237 is brighter and closer; note that the angle between HIP 26268 and HIP 26237 is just 4! 2 .

K 871 is identified by Dreyer with HIP $26235\left(=\theta^{2}\right.$ Ori, $V=$ $5.0, \Delta=1$.7). Our identification $\theta^{1}$ Ori is equally bright and marginally closer (Fig. C.48). Note that $\theta^{1}$ Ori corresponds to HIP 26220 and HIP 26221 (separation 13"). HIP 26224 (at 21", $V=6.7$ ) is also part of $\theta^{1}$, but much fainter.

K 893, near $+6,-10$ in Fig. C.47, is identified by Dreyer with HIP $30700(V=6.5, \Delta=29$ '.5). Our identification is much closer and brighter.

$\mathrm{K} 938$, near $-4.5,+1$ in Fig. C.50) is identified by Dreyer with HIP $31700\left(=v^{3} \mathrm{CMa} ; V=4.4, \Delta=52\right.$ ! $)$ almost a degree north of it. Our identification $v^{2} \mathrm{CMa}$ is brighter and much nearer.

K 959-961. Kepler annotates Has tres trajecit Grünbergerus ad finem Hydrae (Grüberger moves these three to the end of Hydra). Figure C.53 shows the area near K 959 and K960, which includes K975 and K976 (in Hydra). Our preferred identification for each of these is with the nearest HIPPARCOS star in the figure. One problem with this is that we couple the fainter of the pair K960/K976 to the brighter of the pair HIP 49809/HIP 49841. Another problem is the description of K960 as Sequens earundem (the following of the same two [sc. K 959 and K 960]) which appears inapt for a faint star between two brighter ones. Dreyer identifies both K960 and K 976 with HIP 49841, but this has the problem that these two stars have significantly different coordinates and magnitudes in the catalogue. The same problem arises with the identification by Dreyer of both K 961 and K 982 with 9 Crt = HIP 54204 (see Fig. C.54). We prefer to identify K 961 with HIP 54204, and the fainter K 982 with the fainter HIP 54255. Hevelius and Rawlins correct the longitude of $\mathrm{K} 961$, to $\lambda(1601)=113^{\circ} 44^{\prime}$ (by replacing $Z=6$ with $Z=4$ ) which gives a close (0.5) match to HIP 37447 ( $=\alpha$ Mon; see Fig. C.52).

K 975, K 976: see K 959, K 960.

K 982: see K 961.

$\mathrm{K} 1003$, K 1004 both are closest to HIP 67153, near 0, 0 in Fig. C.58, but from the pattern of the whole constellation it is seen that K 1004 is HIP 67669.

M94 is almost identical to M $102=\mathrm{K} 584$.

M 703 is an exact repeat of M $701=\mathrm{K} 339$.

M915 is an almost identical repeat of M $908=\mathrm{K} 908$.

\section{Appendix C: Figures}

To illustrate and clarify our identifications we provide figures for each constellation. It should be noted that the following equations are used only for the figures, i.e. for illustrative purposes: to compute the angles between positions, e.g. to find the nearest counterpart, we always use Eqs. (1) and (2).

In these figures the stars listed with the constellation in Progym are shown red, those added in Kepler in blue, and other stars listed in Progym and Kepler are shown light-red and lightblue, respectively. For all stars we use positions from KeplerE. In yellow we indicate stars from Secunda Classis (which are discussed in Verbunt \& Van Gent, Paper III, in preparation).

To minimize deformation of the constellations, we determine the center of the constellation $\lambda_{\mathrm{c}}, \beta_{\mathrm{c}}$ from the extremes in $\lambda$ and $\beta$, compute the rotation matrix

$\mathcal{R} \equiv\left(\begin{array}{ccc}\cos \lambda_{\mathrm{c}} \cos \beta_{\mathrm{c}} & \sin \lambda_{\mathrm{c}} \cos \beta_{\mathrm{c}} & \sin \beta_{\mathrm{c}} \\ -\sin \lambda_{\mathrm{c}} & \cos \lambda_{\mathrm{c}} & 0 \\ -\cos \lambda_{\mathrm{c}} \sin \beta_{\mathrm{c}} & -\sin \lambda_{\mathrm{c}} \sin \beta_{\mathrm{c}} & \cos \beta_{\mathrm{c}}\end{array}\right)$

which moves this center to $(x, y, z)=(1,0,0)$, and then apply this rotation to each of the stellar positions $\lambda_{i}, \beta_{i}$ :

$$
\left(\begin{array}{l}
x \\
y \\
z
\end{array}\right)=\mathcal{R}\left(\begin{array}{c}
\cos \lambda_{i} \cos \beta_{i} \\
\sin \lambda_{i} \cos \beta_{i} \\
\sin \beta_{i}
\end{array}\right)
$$

The resulting $y, z$ values correspond roughly to differences in longitude and latitude, exact at the center $\lambda_{\mathrm{c}}, \beta_{\mathrm{c}}$ and increasingly deformed away from the center. We plot the rotated positions of the stars in KeplerE as $d \lambda \equiv y$ and $d \beta \equiv z$ with filled circles. The same rotation matrix $\mathcal{R}$ is applied to all stars down to a magnitude limit $V_{m}$ (usually $V_{m}=6.0$ ) from the Hipparcos Catalogue and those in the field of view are plotted as open circles. The symbol sizes are determined from the magnitudes as indicated in the legenda. The values used for $\lambda_{\mathrm{c}}, \beta_{\mathrm{c}}$ and $V_{m}$ are indicated with each figure.

Where necessary we show enlarged detail figures; for easy comparison with the figures showing the whole constellation, these detail figures use the same rotation center (and thus rotation matrix). 
F. Verbunt and R. H. van Gent: Three editions of the star catalogue of Tycho Brahe

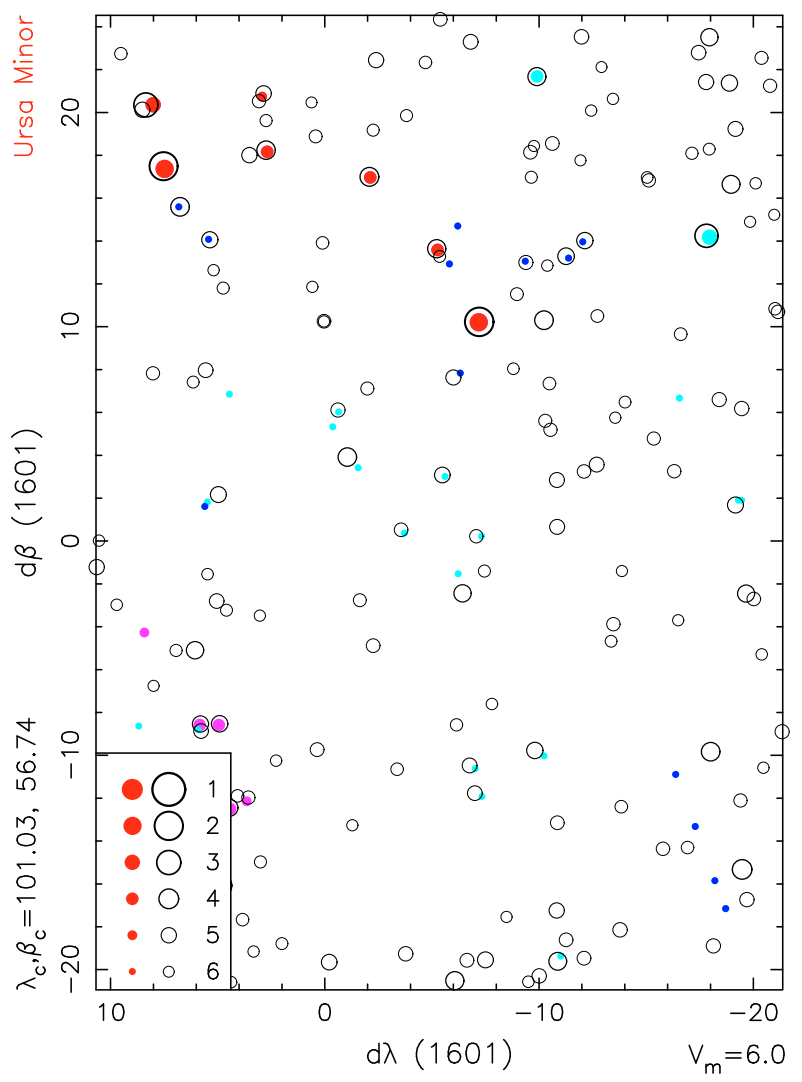

Fig. C.1. Ursa Minor. The stars additional to the 7 stars given in Progym include four stars in Camelopardalis (below right) with no close counterparts, and one star (near $+5.5,1.5$ ) also listed in Ursa Maior. We agree with Dreyer in not finding a suitable identification for K 15 (near $-6,14.5$ ).

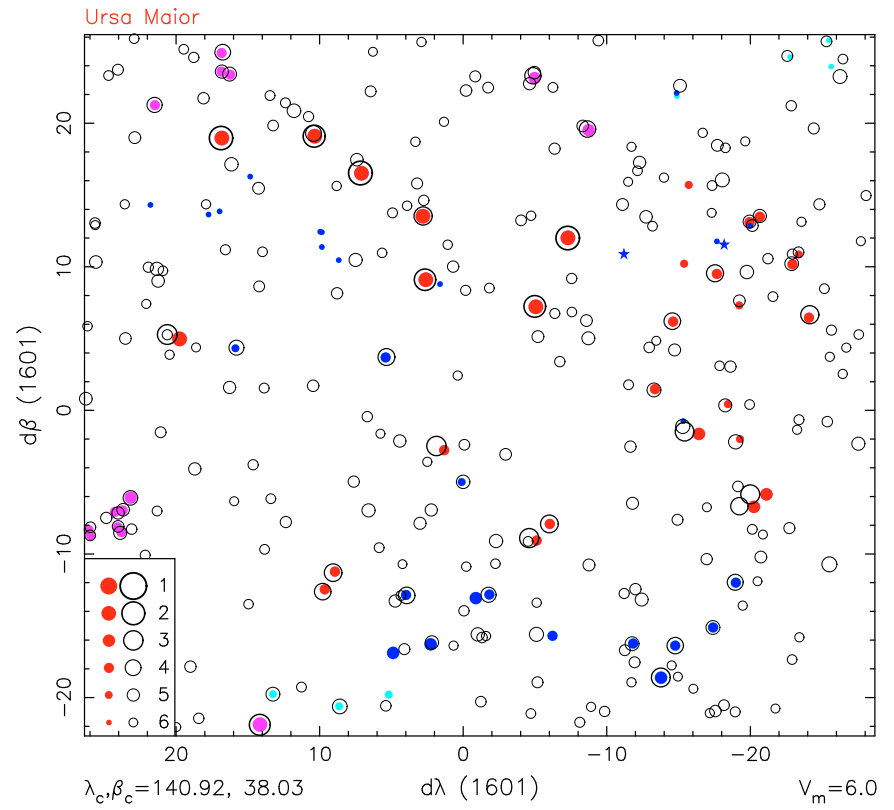

Fig. C.2. Ursa Maior. K 67 and K 88 at the positions given in Kepler are indicated $\star$. See also Fig. C.3.

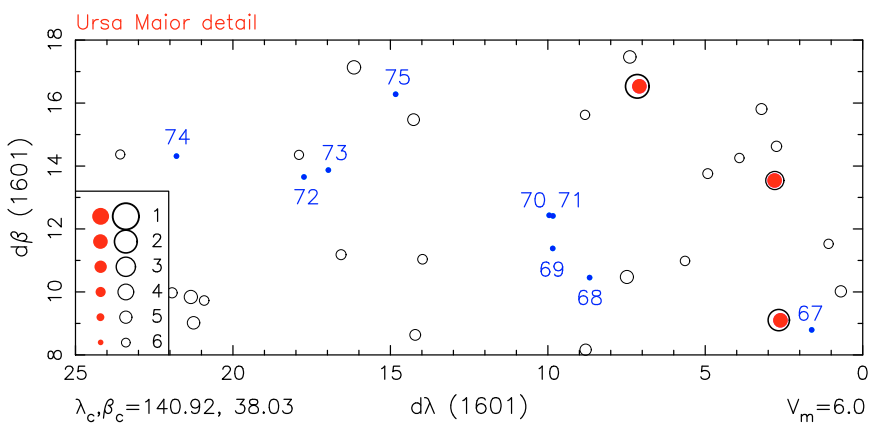

Fig. C.3. Ursa Maior detail. No good counterparts are found for K 67-75 even if the search is extended down to $V=6.5$. Rawlins (1993) notes that Brahe's positions in this area are in "extreme confusion”. Compare with Fig. C.2.

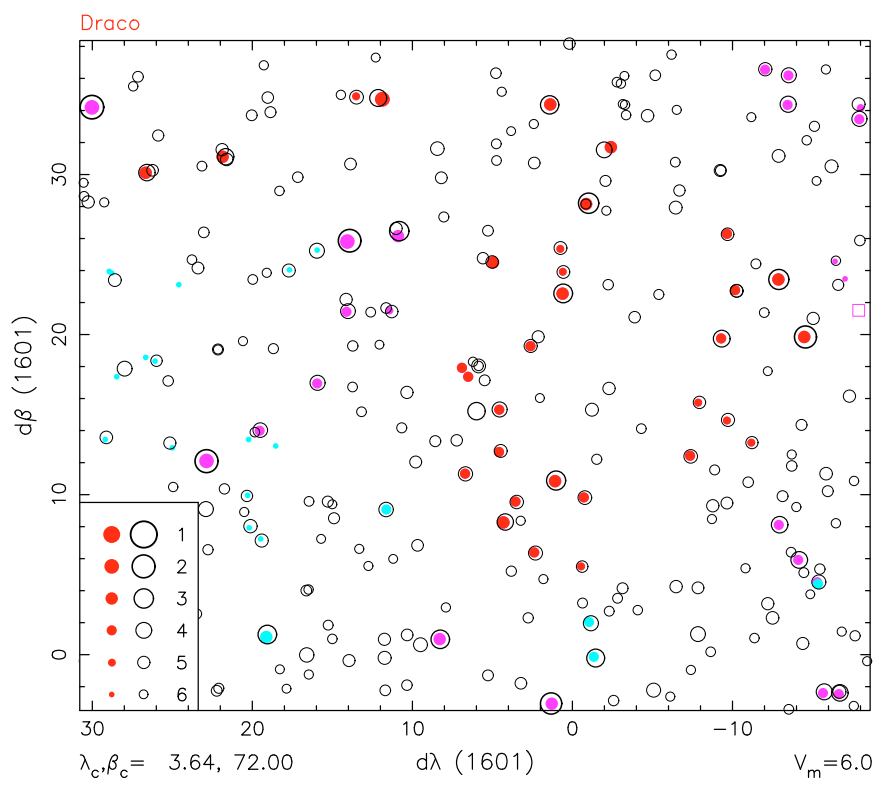

Fig. C.4. Draco.

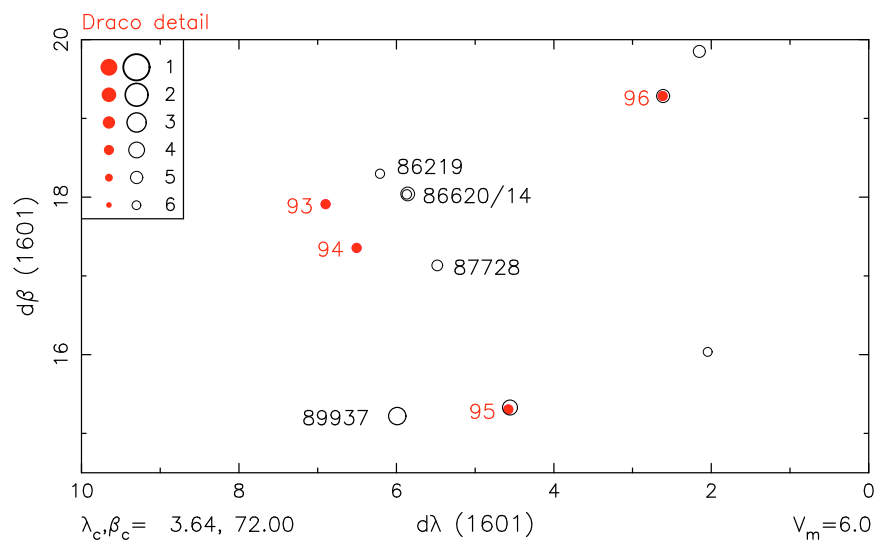

Fig. C.5. Draco detail. K 93,94. We identify K 93 with the close pair HIP 86614/86620 $\left(=\psi^{1}\right.$ Dra $)$ and K 94 with HIP 87728. For K 94 Dreyer (1916) and Rawlins (1993) prefer HIP 89937 (= $\chi$ Dra) as counterpart. 
A\&A 516, A28 (2010)

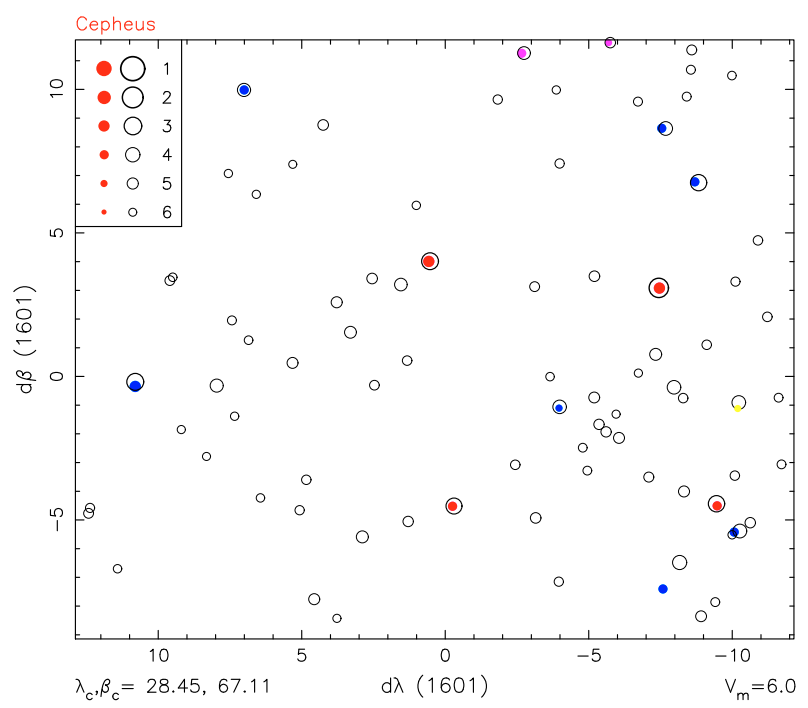

Fig. C.6. Cepheus.

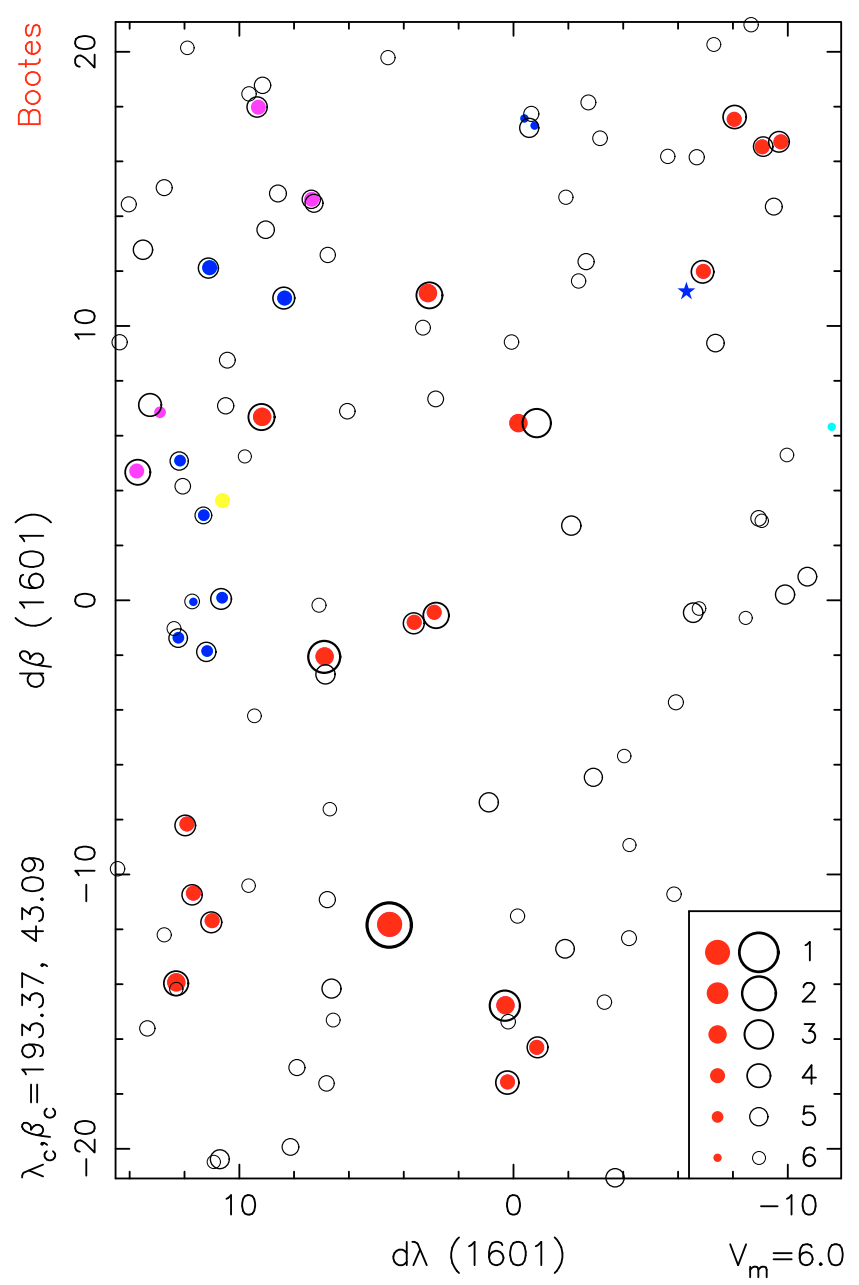

Fig. C.7. Bootes. The position of K 145 in Kepler is indicated $\star$.

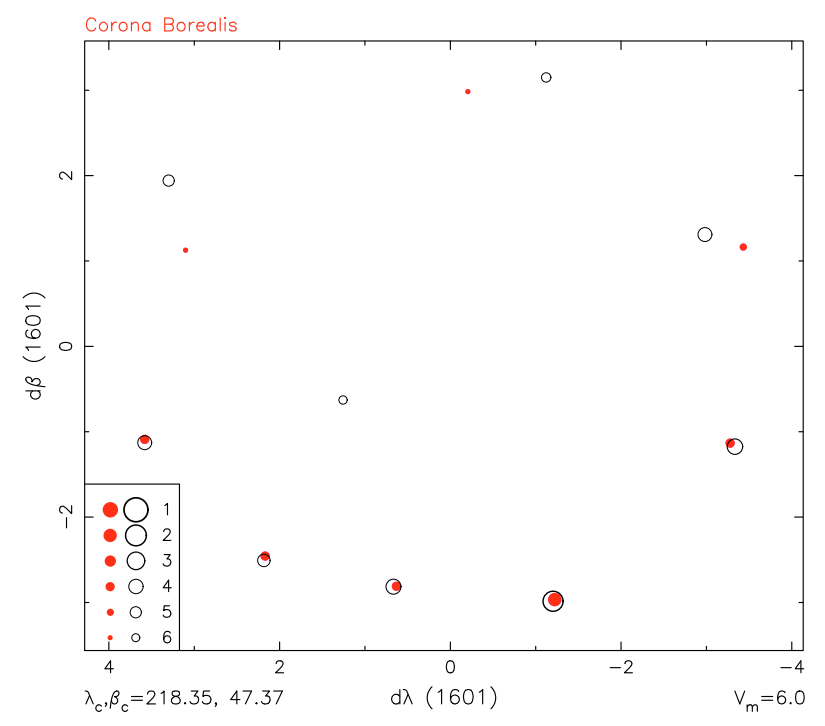

Fig. C.8. Corona Borealis.

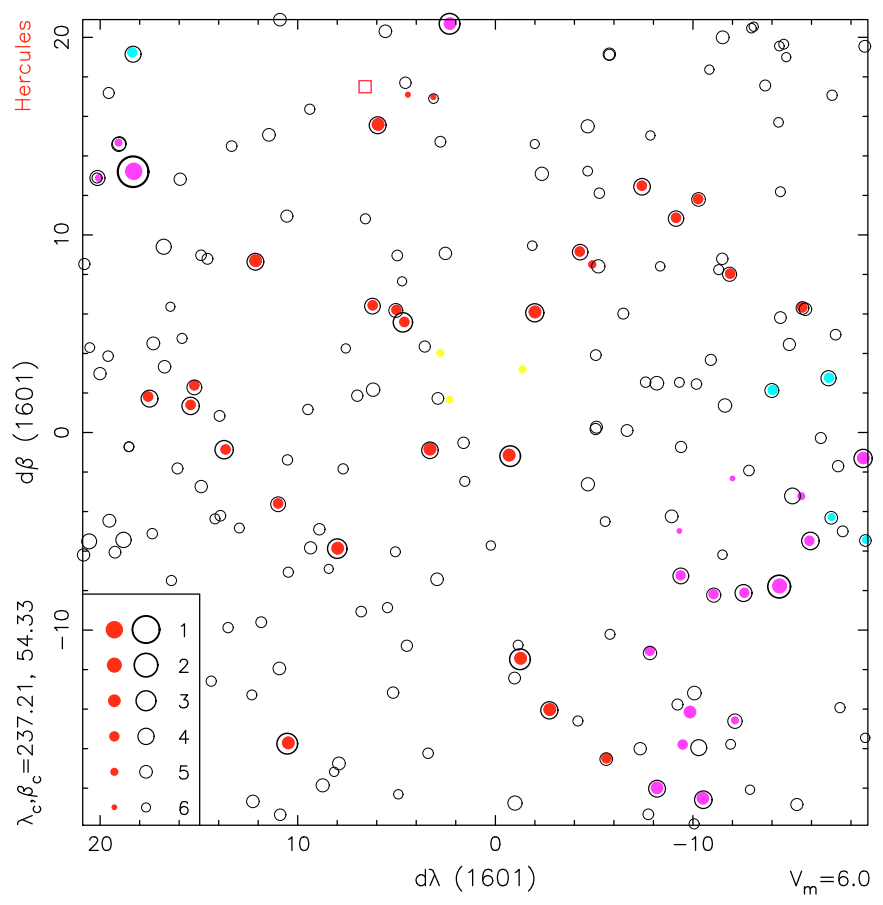

Fig. C.9. Hercules. The star from Secunda Classis near -1.4, 3.2 may be identified with M 13 . 
F. Verbunt and R. H. van Gent: Three editions of the star catalogue of Tycho Brahe

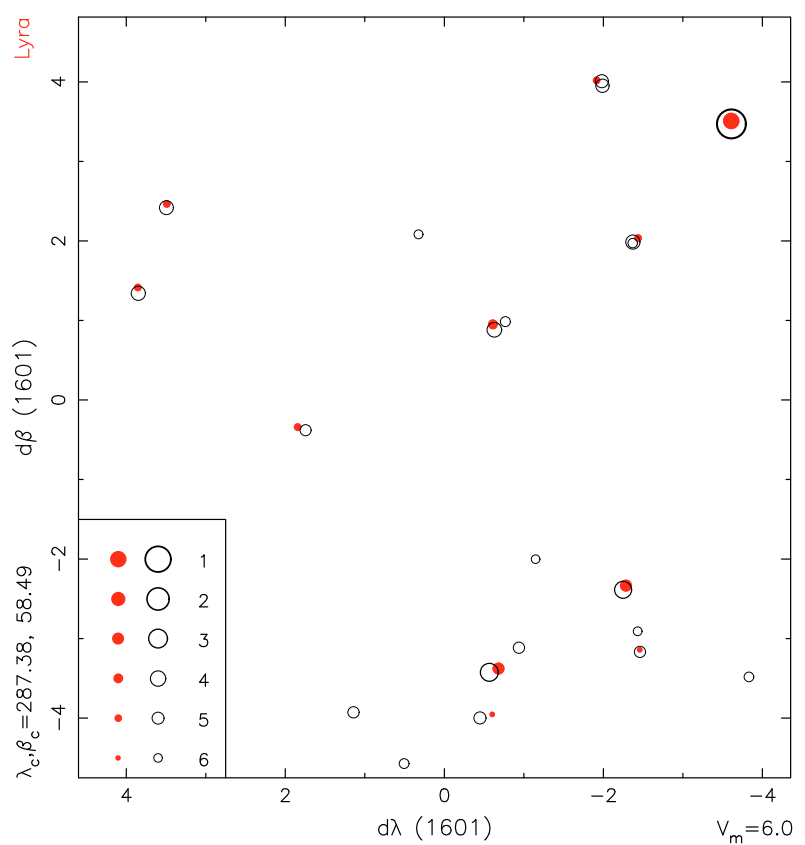

Fig. C.10. Lyra.

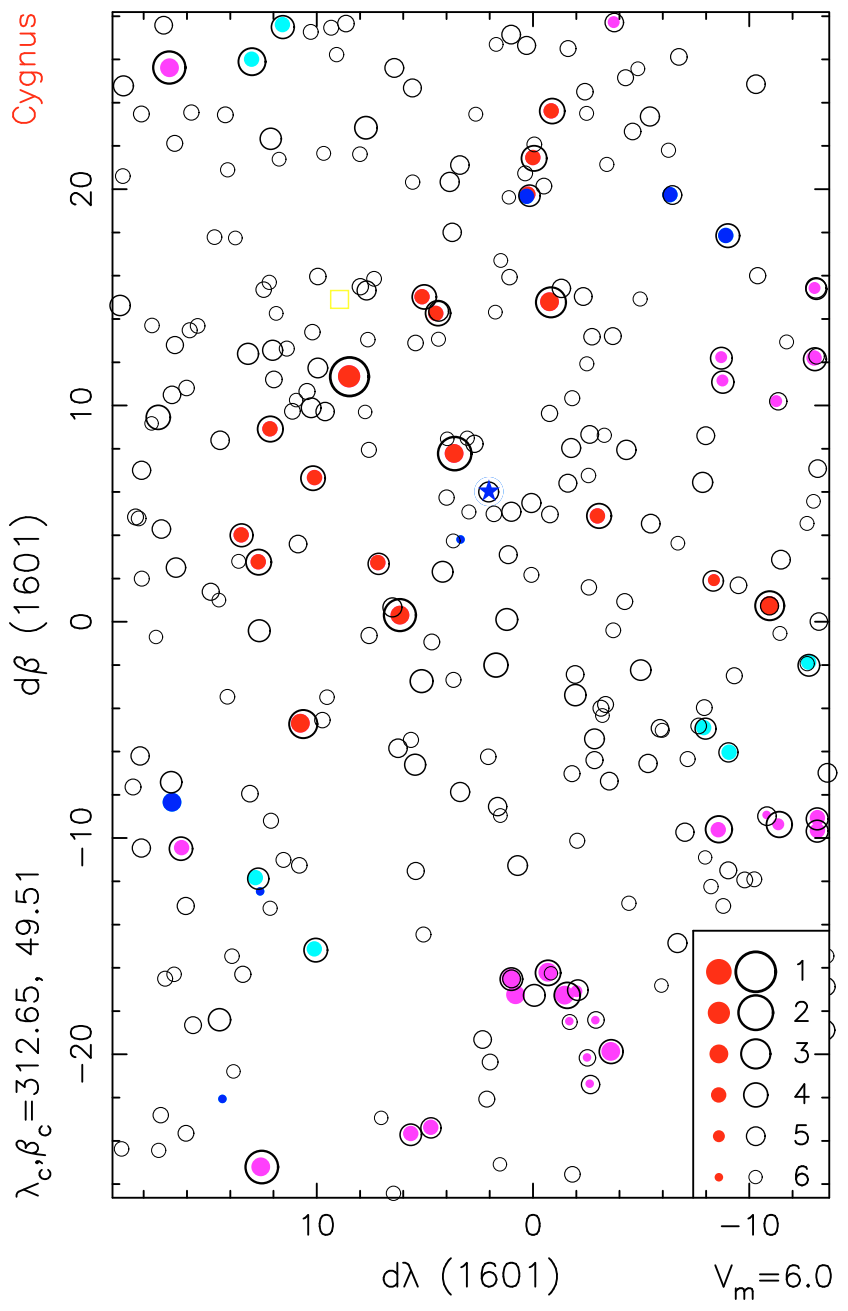

Fig. C.11. Cygnus. Nova 1600 aka P Cygni $=$ HIP 100044 is indicated with $\star$.

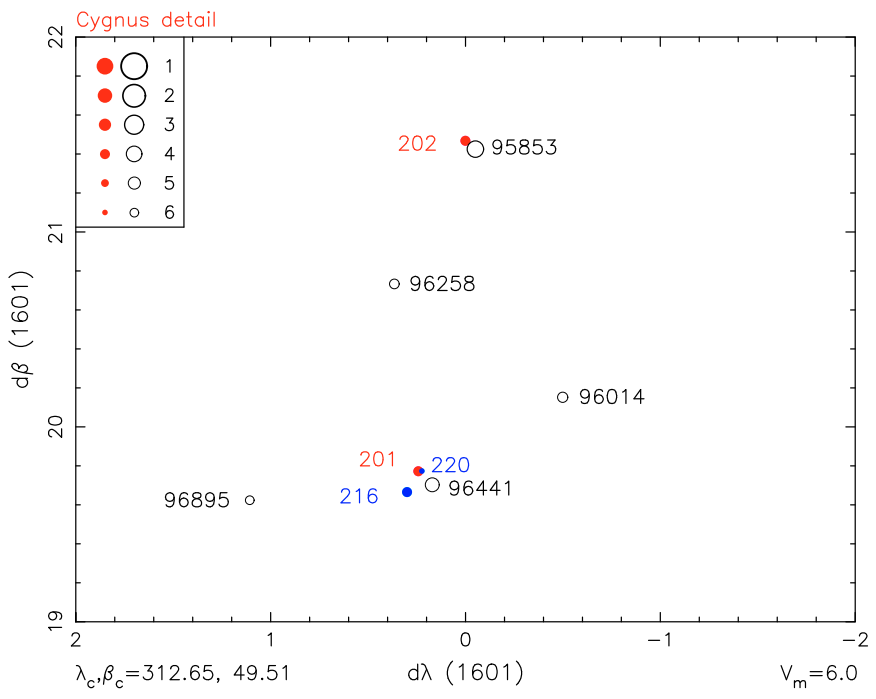

Fig. C.12. Detail of Cygnus illustrating that K 201, K 216 and K 220 are repeated entries for the same star.

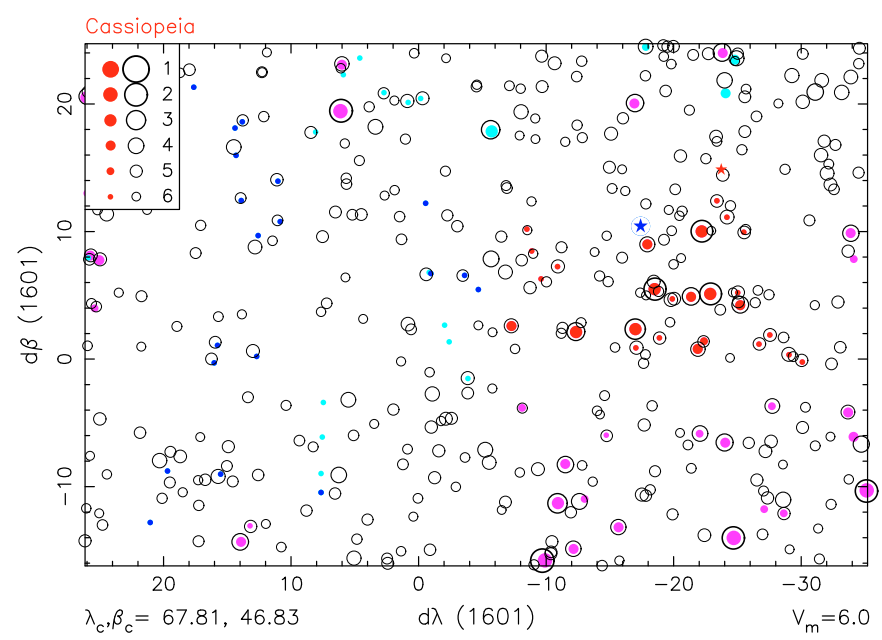

Fig. C.13. Cassiopeia. The blue $\star$ indicates Nova 1572. The red star indicates the position of $\mathrm{K} 238$ in Kepler.

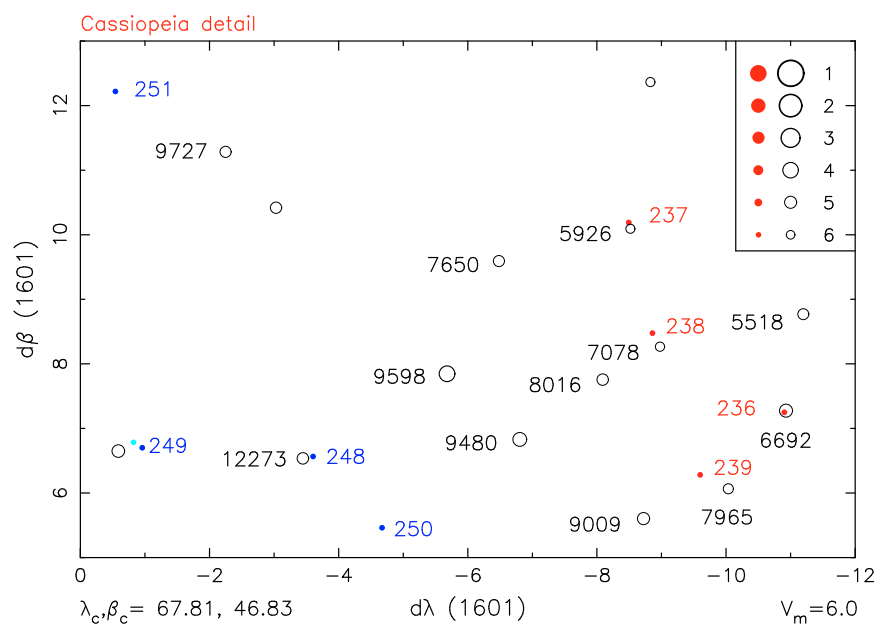

Fig. C.14. Cassiopeia detail, illustrating the identifications by Dreyer (1916) and Rawlins (1993) of K 237-239 with HIP 9598, HIP 9480 and HIP 9009. 


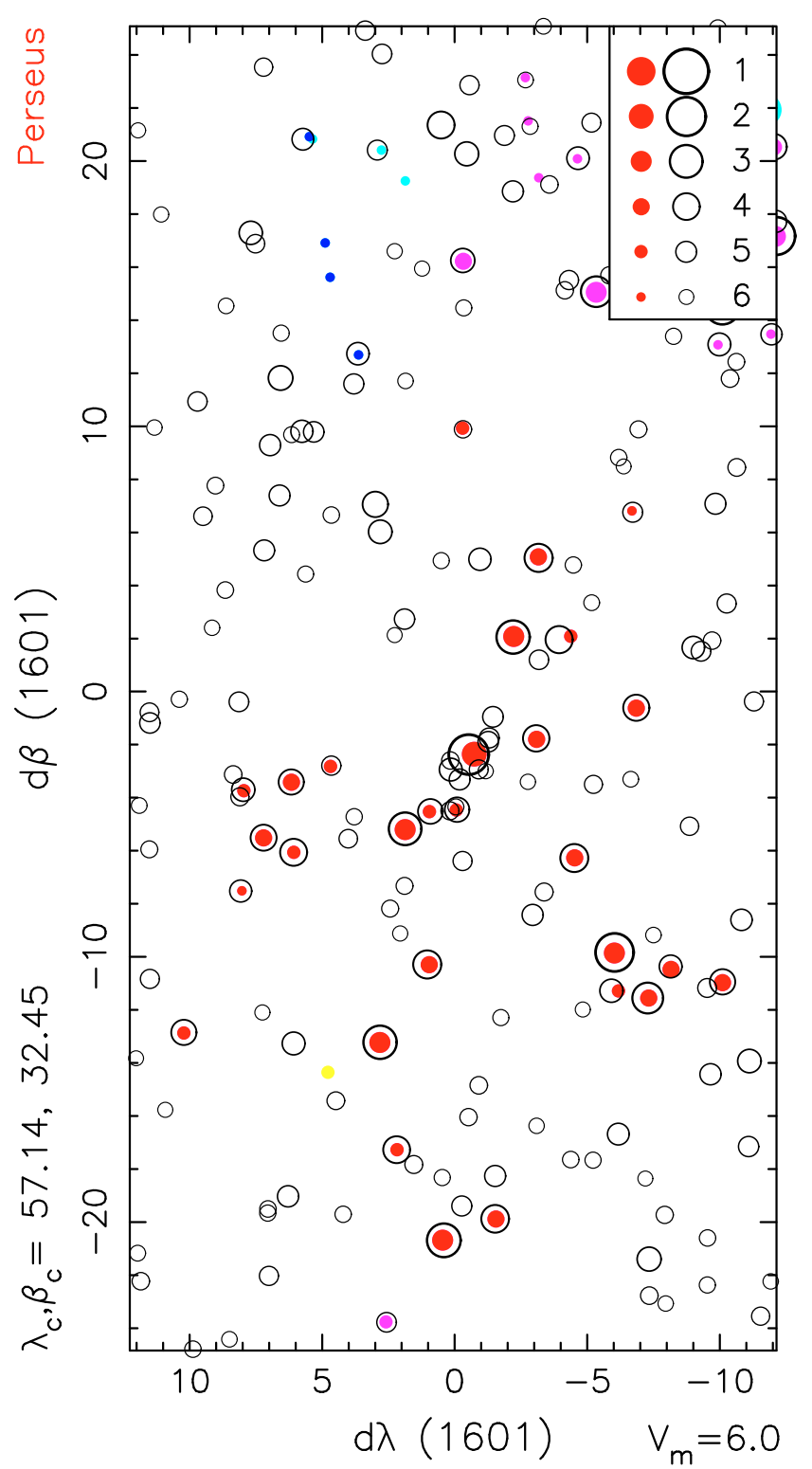

Fig. C.15. Perseus. The additional stars (in blue) appear shifted from a better match about $10^{\circ}$ south.

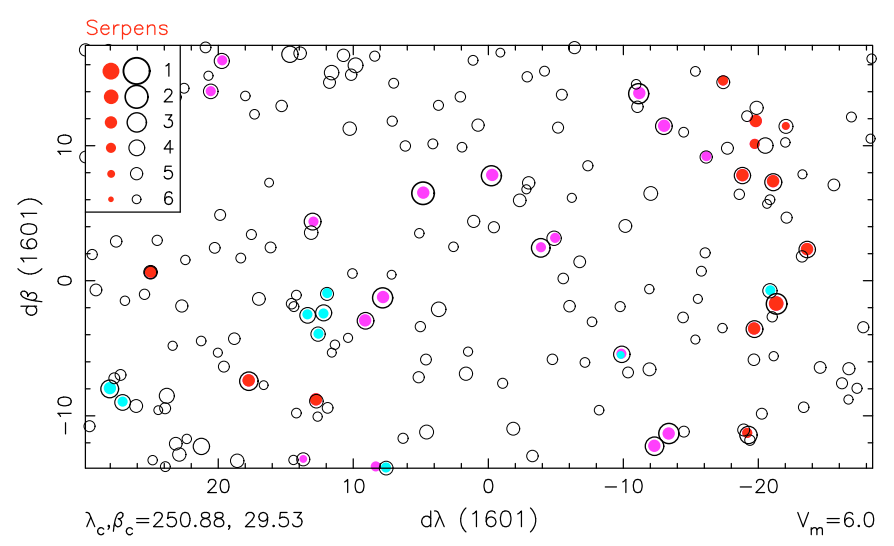

Fig. C.16. Serpens.

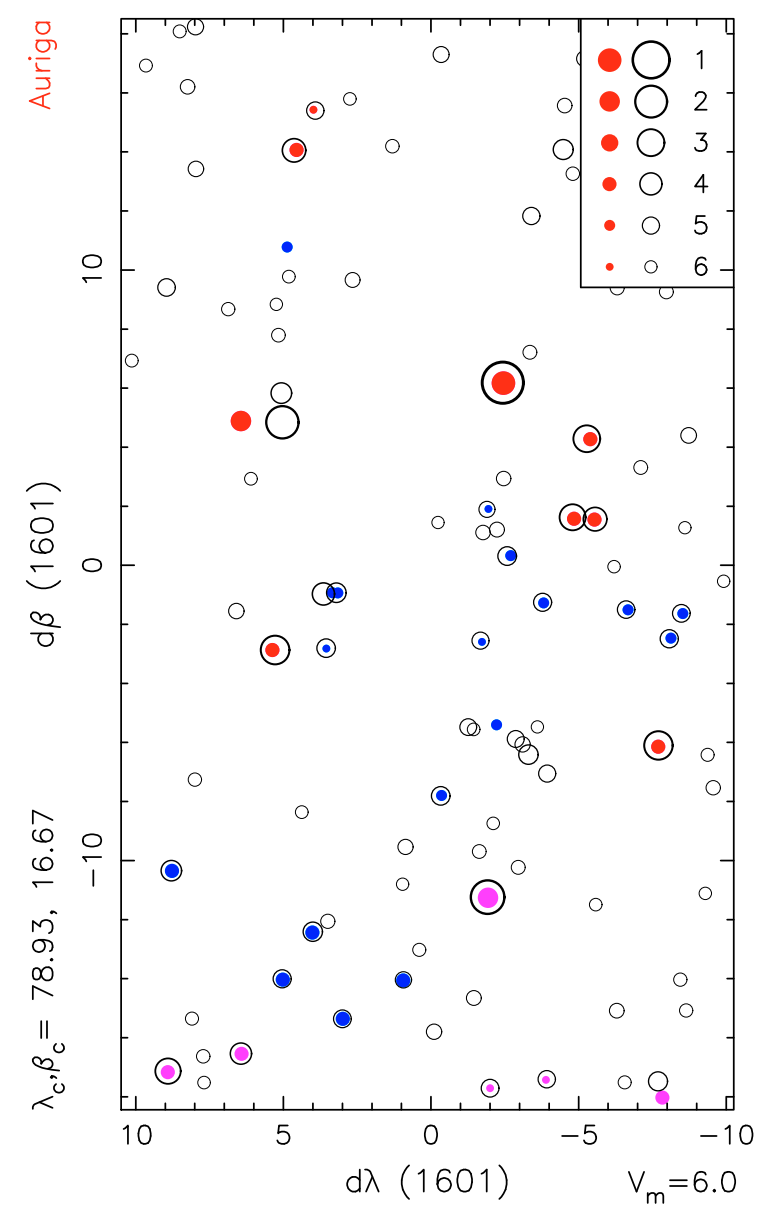

Fig. C.17. Auriga.

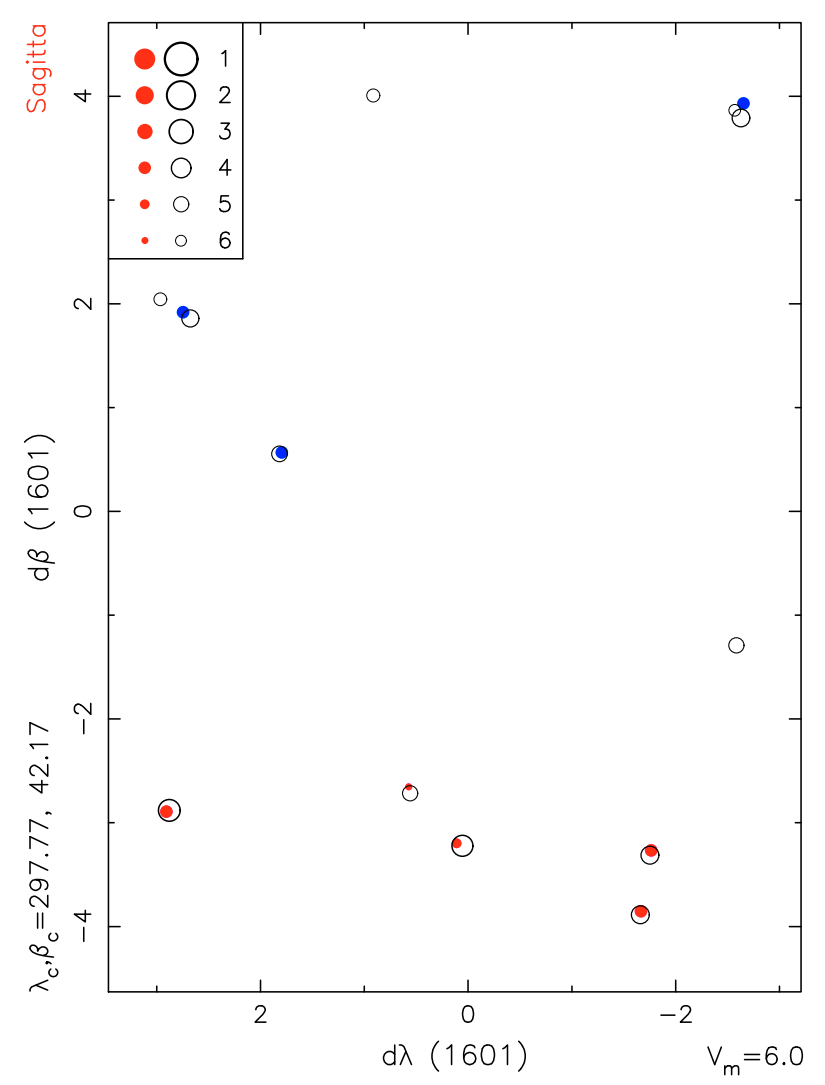

Fig. C.18. Sagitta. 
F. Verbunt and R. H. van Gent: Three editions of the star catalogue of Tycho Brahe

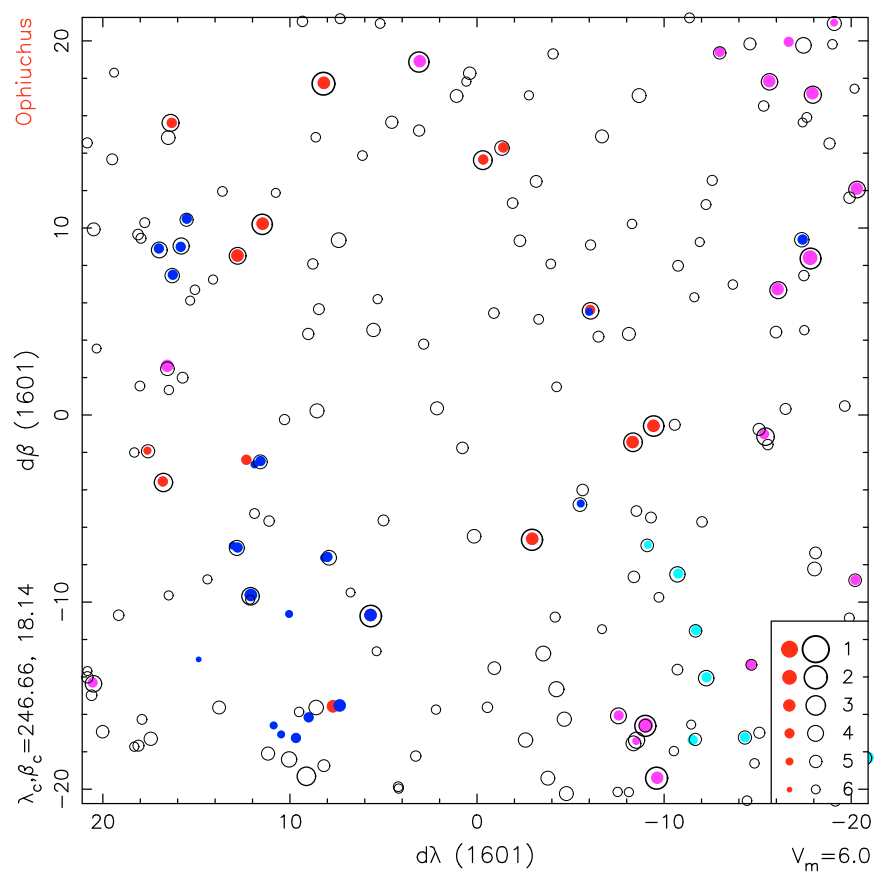

Fig. C.19. Ophiuchus.

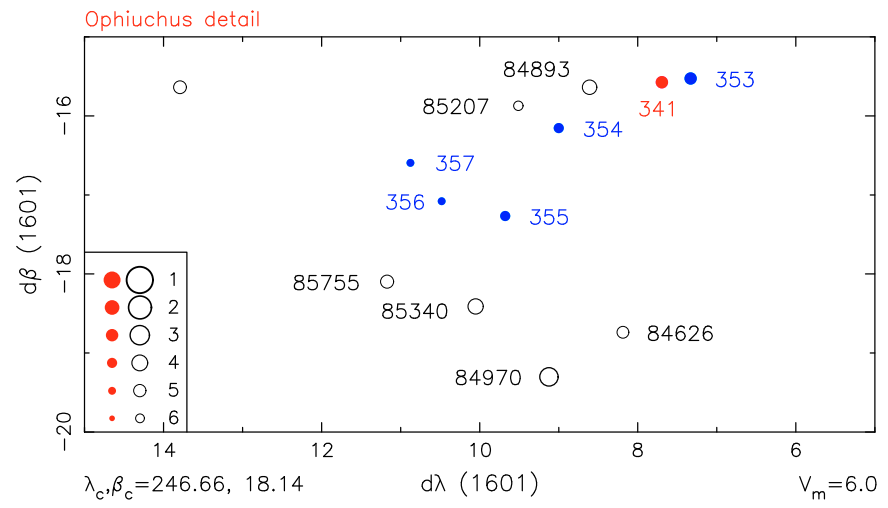

Fig. C.20. Ophiuchus detail.

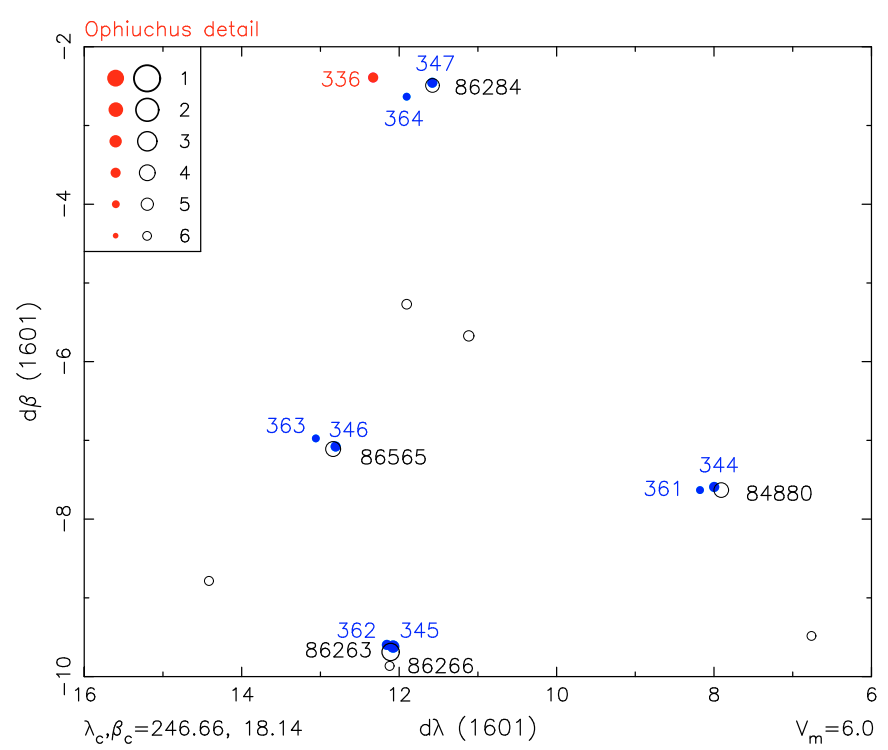

Fig. C.21. Ophiuchus detail.

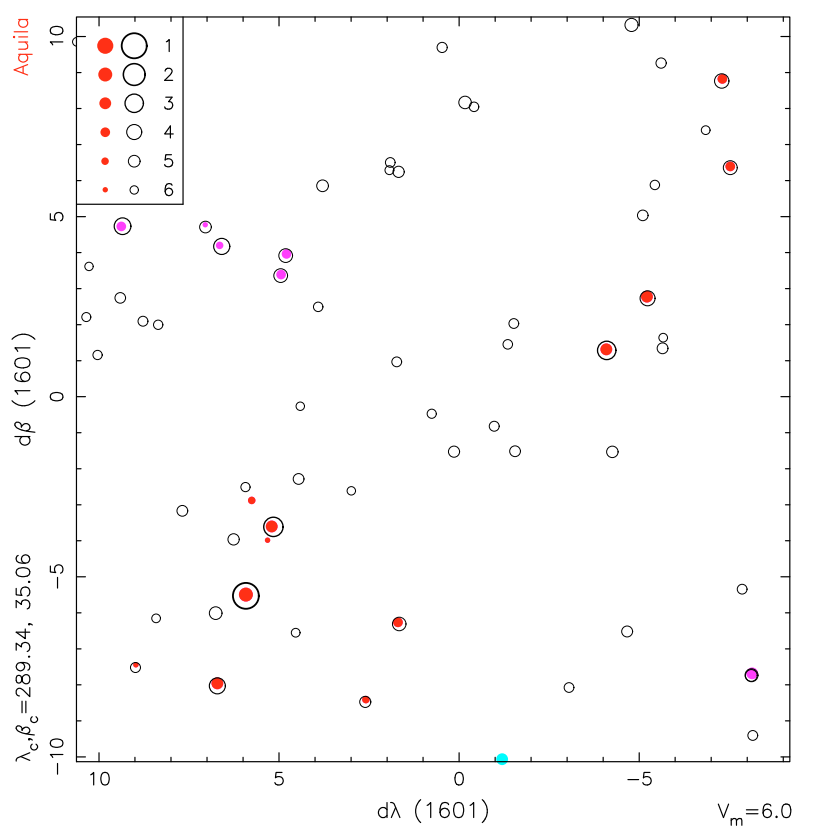

Fig. C.22. Aquila.

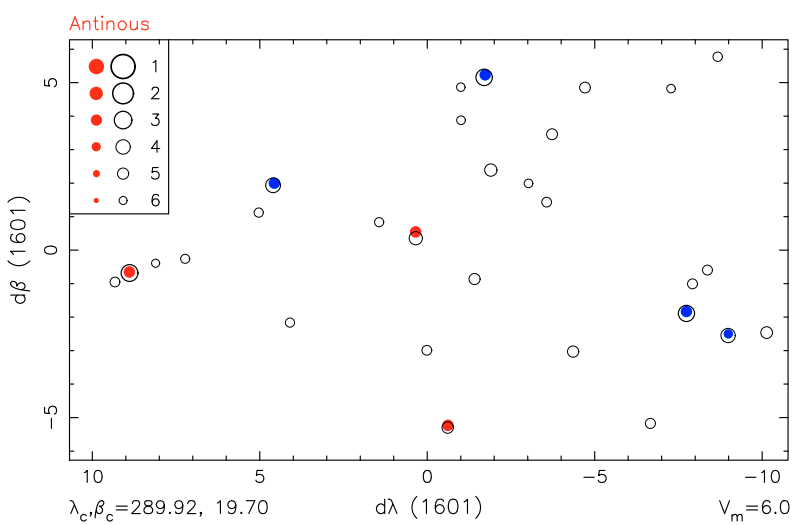

Fig. C.23. Antinous.

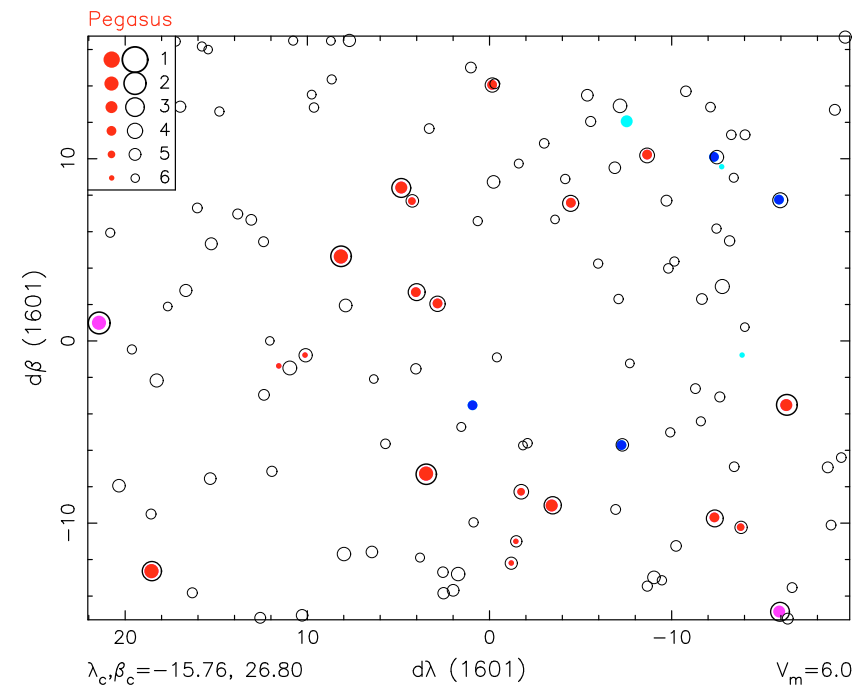

Fig. C.24. Pegasus. 
A\&A 516, A28 (2010)

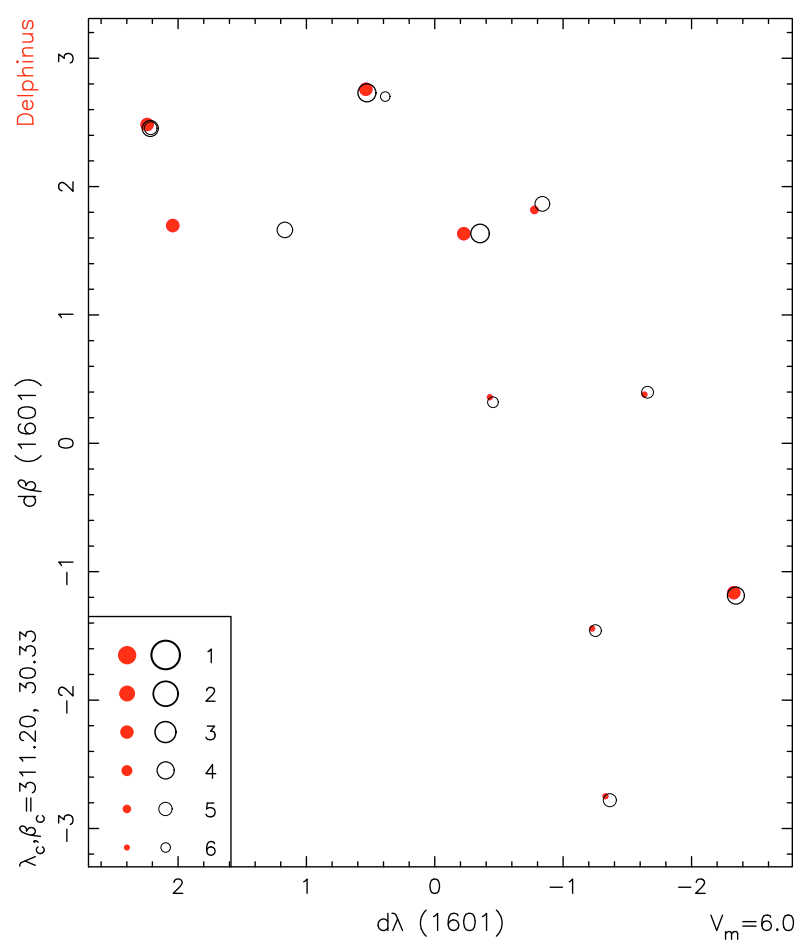

Fig. C.25. Delphinus.

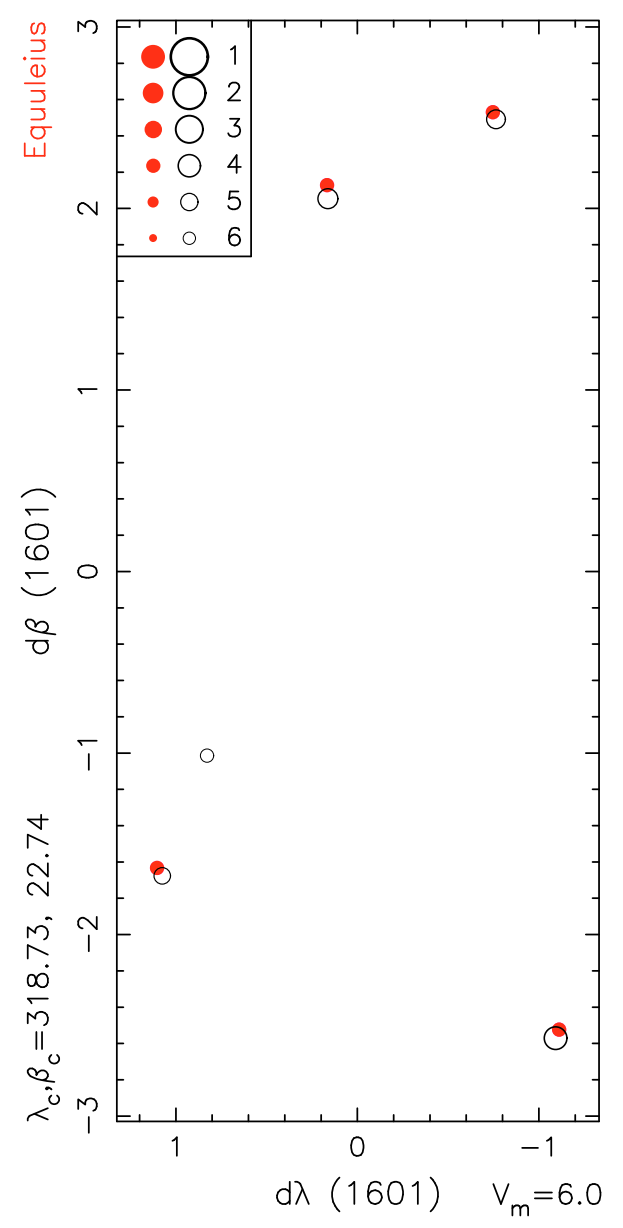

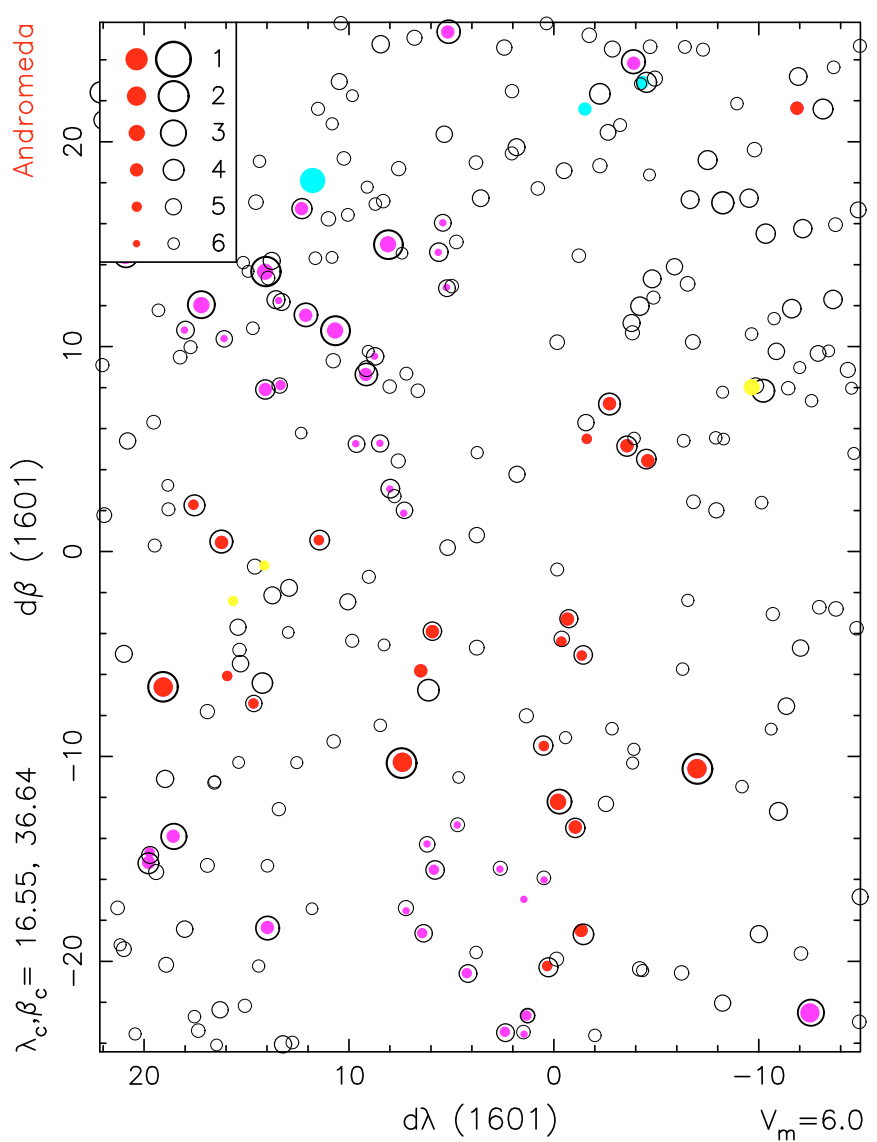

Fig. C.27. Andromeda.

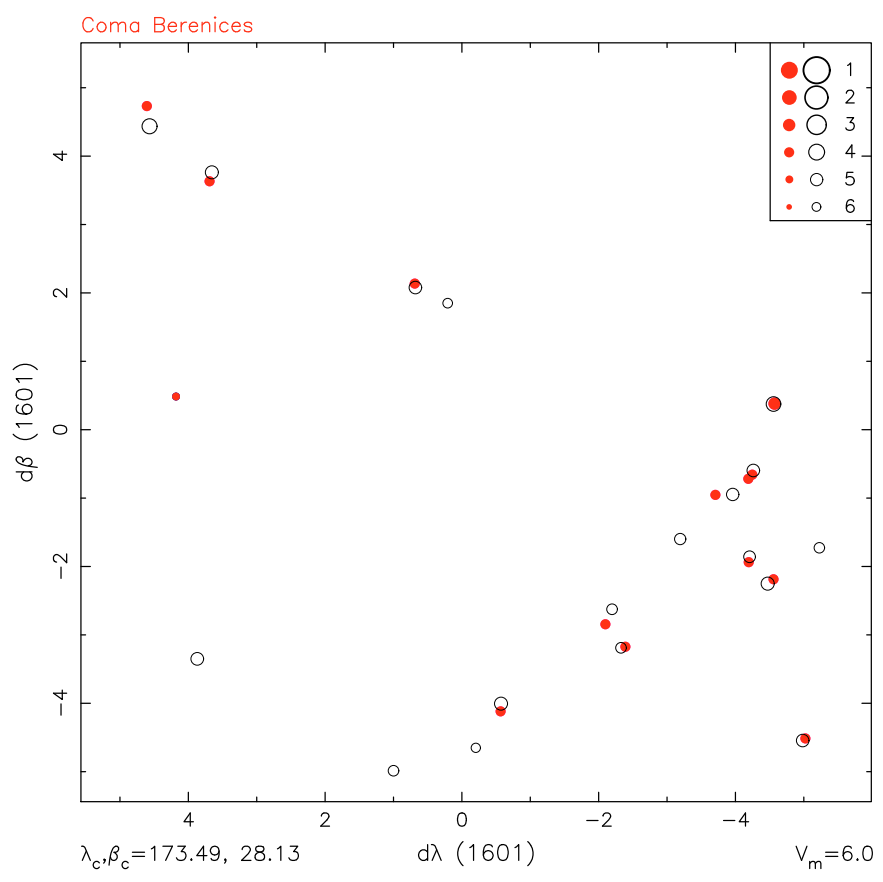

Fig. C.28. Coma Berenices.

Fig. C.26. Equuleus. 
F. Verbunt and R. H. van Gent: Three editions of the star catalogue of Tycho Brahe

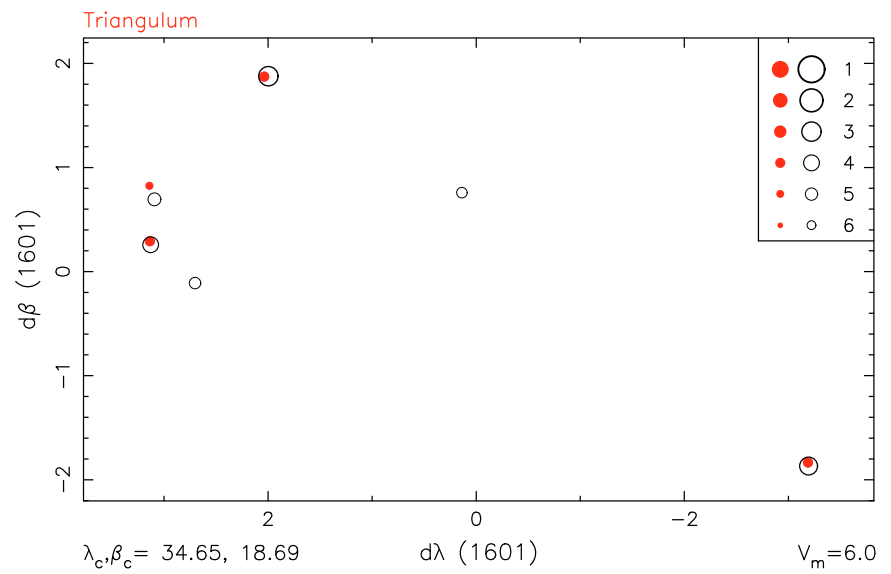

Fig. C.29. Triangulum.

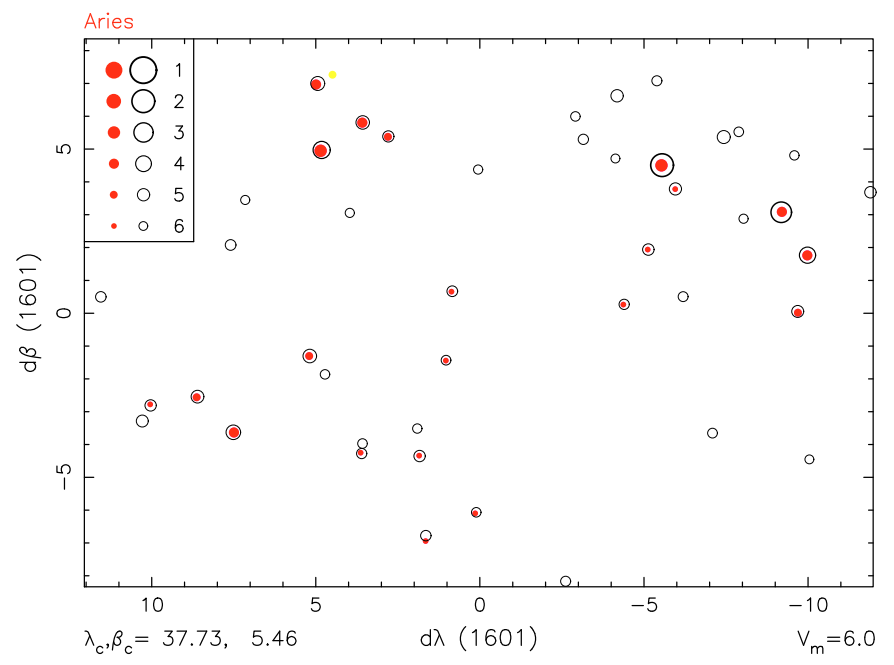

Fig. C.30. Aries.

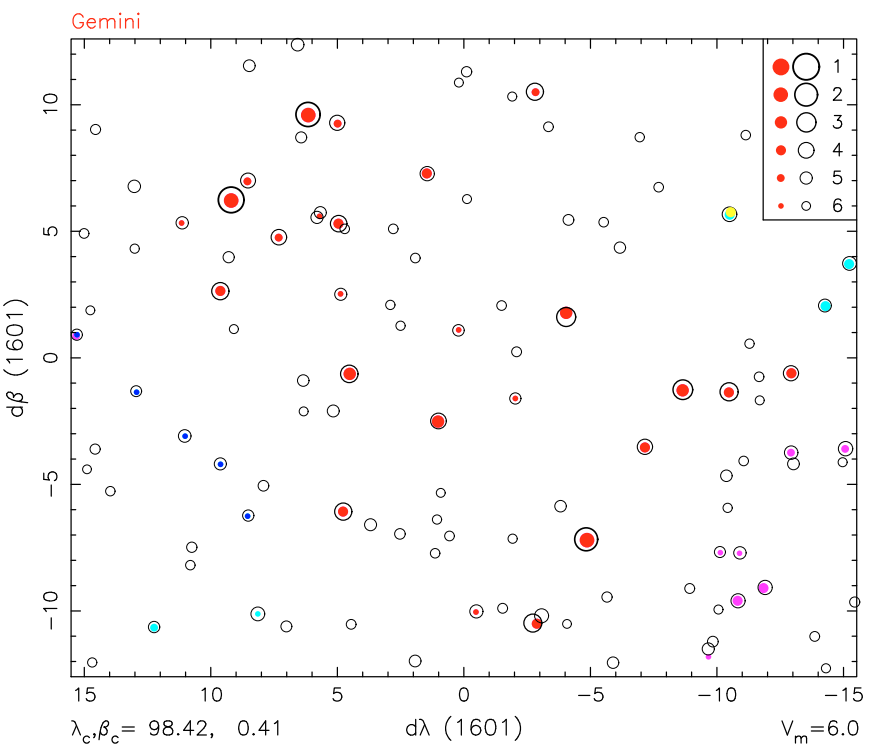

Fig. C.31. Gemini.

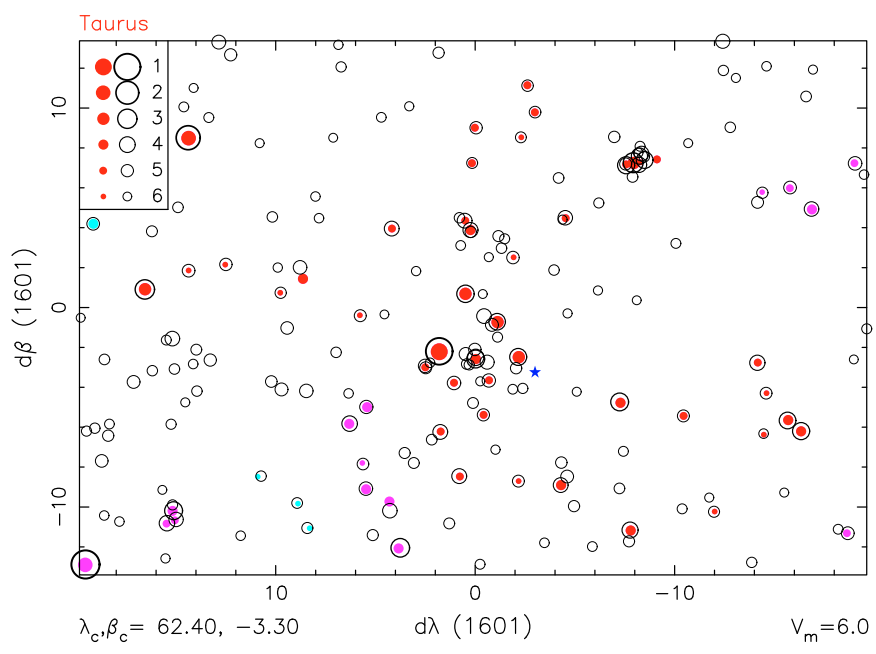

Fig. C.32. Taurus. The Kepler position of K 547, indicated $\star$, has no good identification. The emended position at about $-3,+10$ identifies it with HIP 19171.

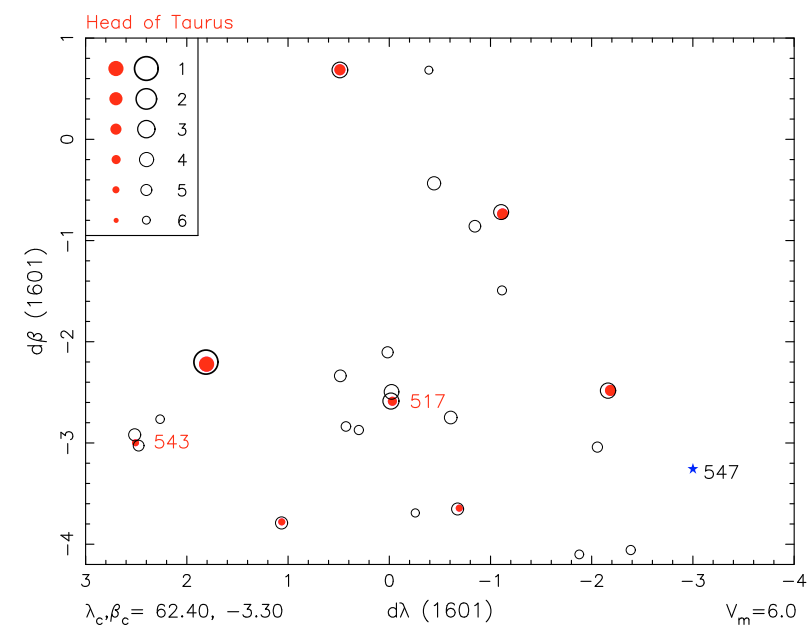

Fig. C.33. Taurus: enlarged detail of the head, showing K 517 to be best identified with $\theta^{2}$ the southern star of the close pair $\theta^{1} / \theta^{2}$, and $\mathrm{K} 543$ to be between two posible Hipparcos counterparts. The Kepler position of K 547 , indicated $\star$, has no good identification.

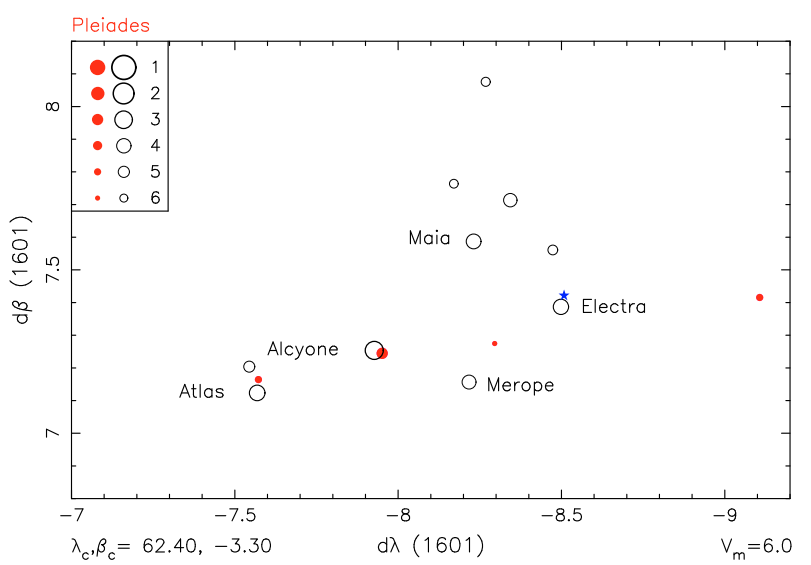

Fig. C.34. Taurus: enlarged detail of the Pleiades. The position of K 534 in Manuscript and Progym is indicated with a blue $\star$, and shows that this star should be identified with Electra. 


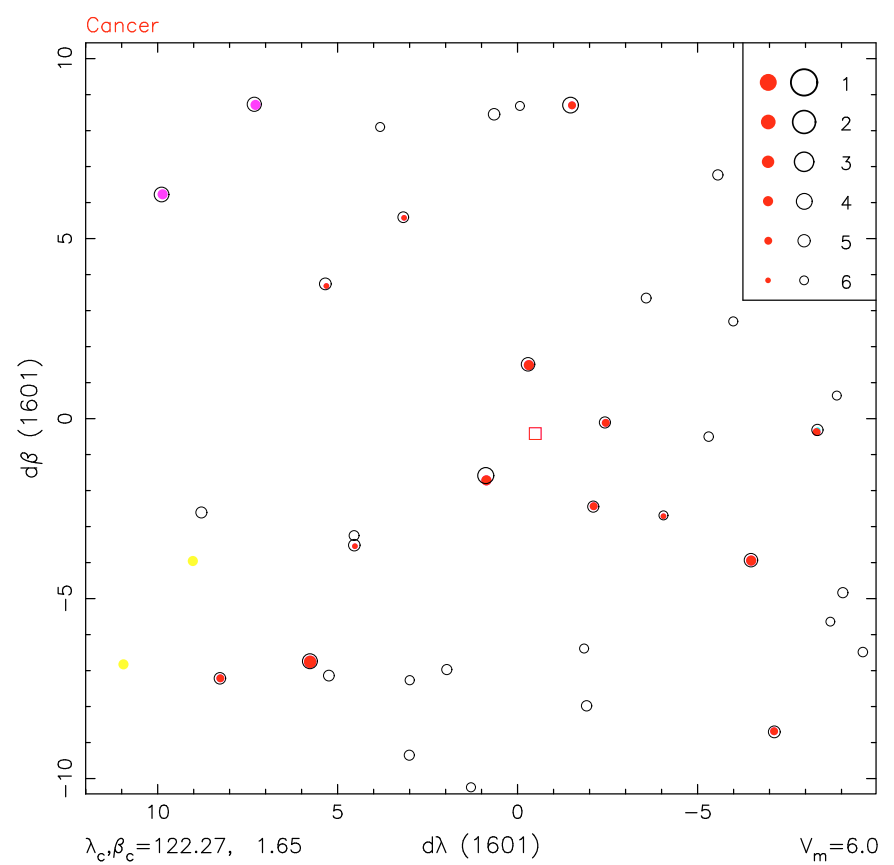

Fig. C.35. Cancer. The red square indicates K 577, Praesepe.

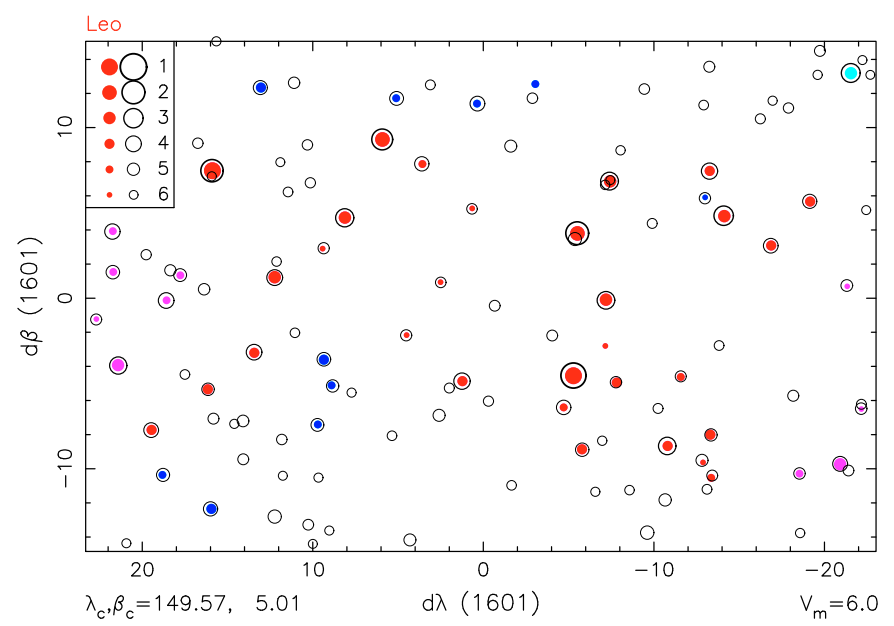

Fig. C.36. Leo.

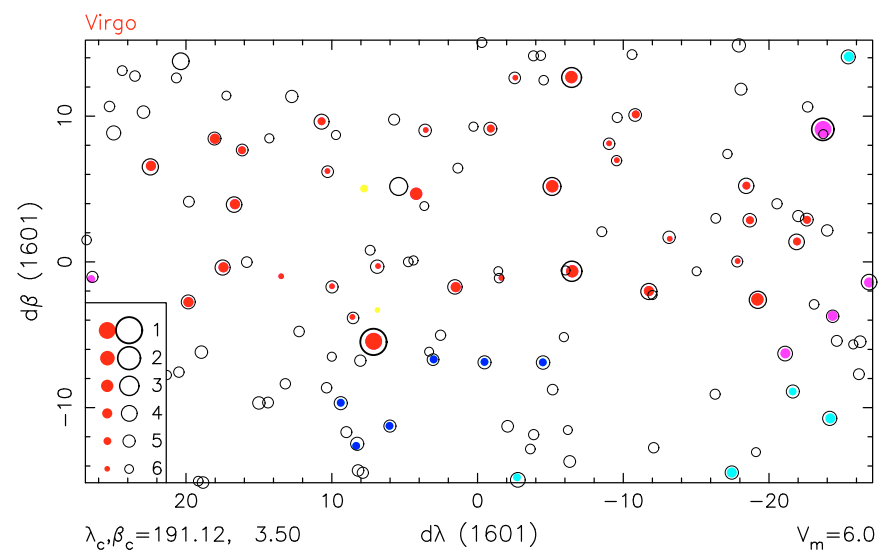

Fig. C.37. Virgo.

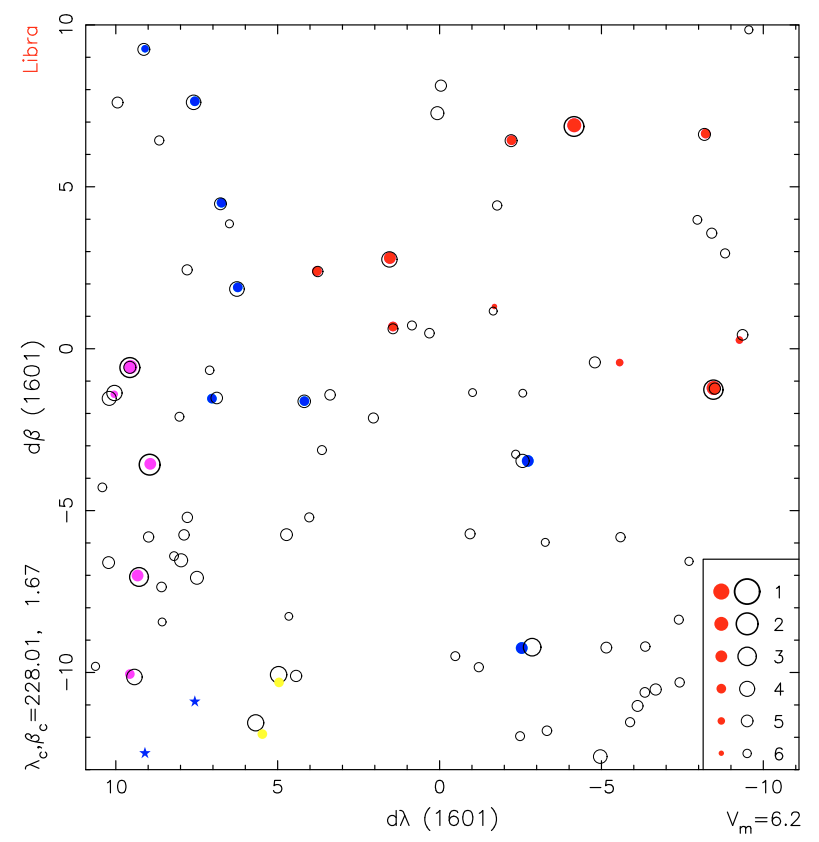

Fig. C.38. Libra. The position as given in Kepler for K 685,686 are indicated with $\star$ and have no near counterparts. The emended positions are in the upper left corner.

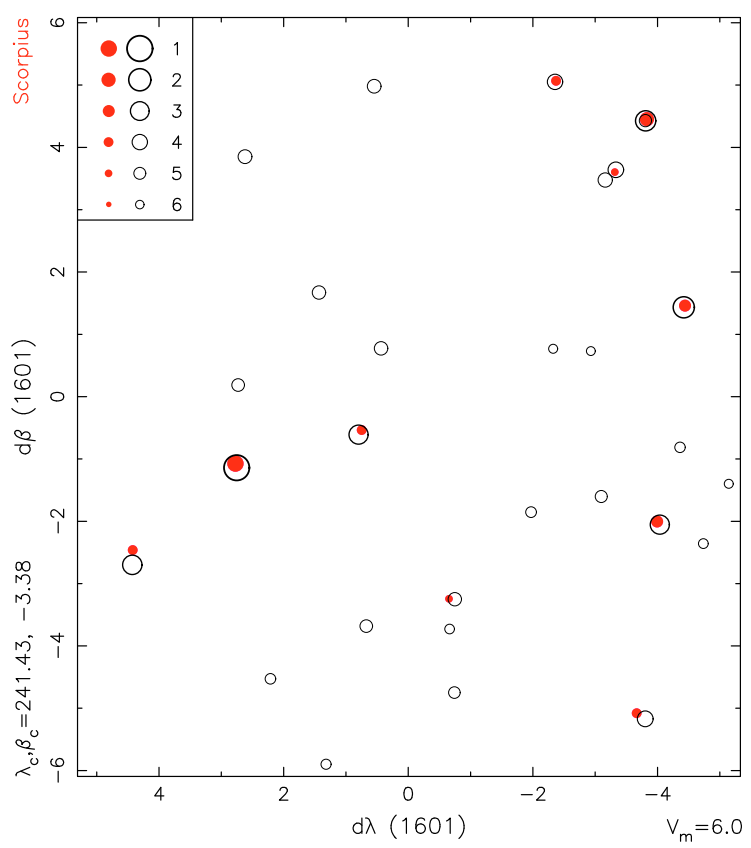

Fig. C.39. Scorpius. Only the northern stars are in Brahe's catalogue.

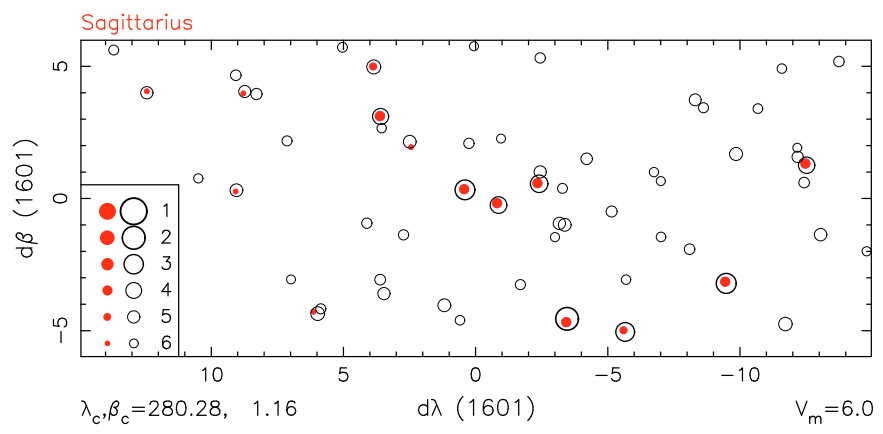

Fig. C.40. Sagittarius. 
F. Verbunt and R. H. van Gent: Three editions of the star catalogue of Tycho Brahe

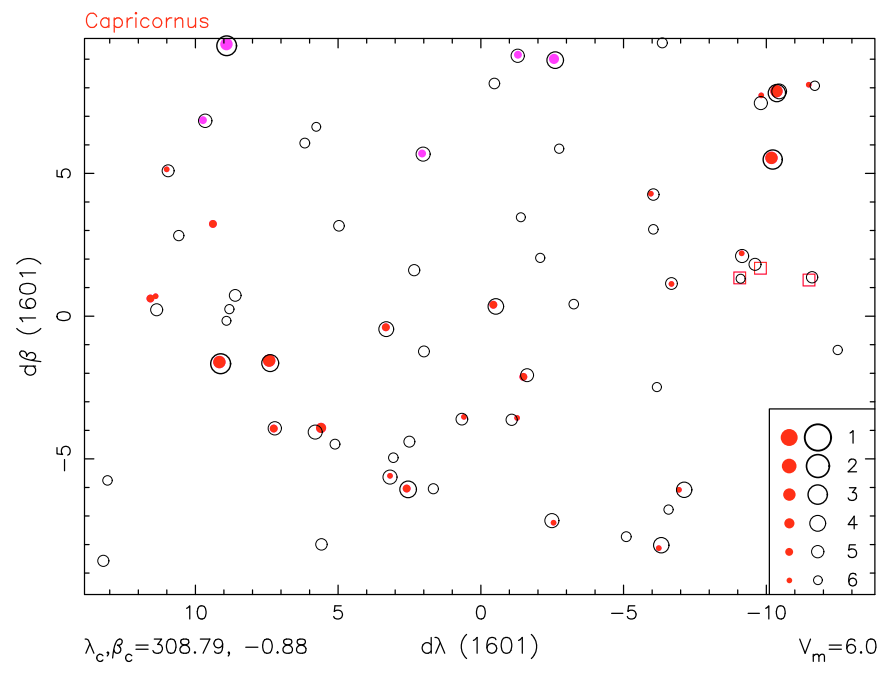

Fig. C.41. Capricornus. Three stars listed as nebulous by Brahe, indicated with $\square$, are identified with ordinary stars.

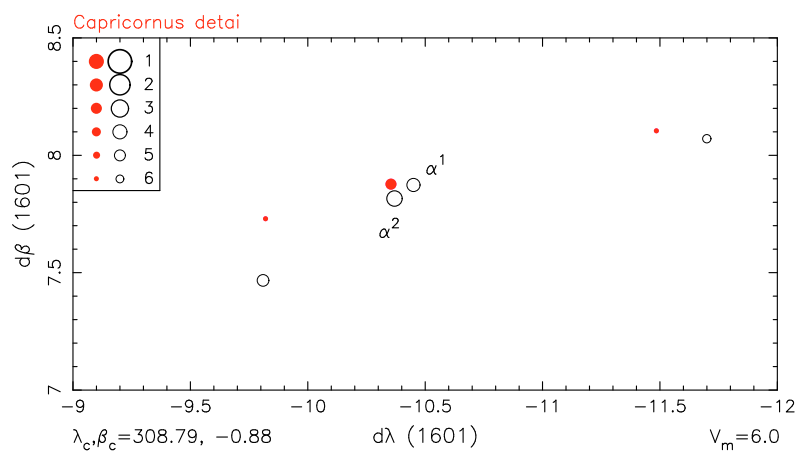

Fig. C.42. Capricornus detail, showing identification of K713 with $\alpha^{2}$ Cap.

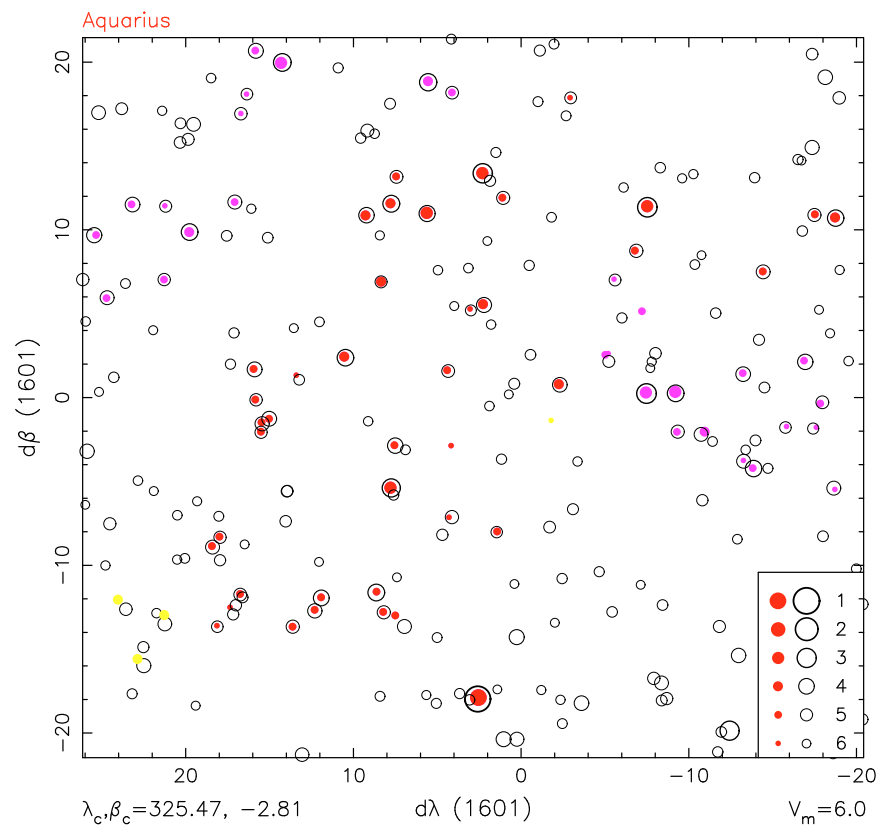

Fig. C.43. Aquarius.

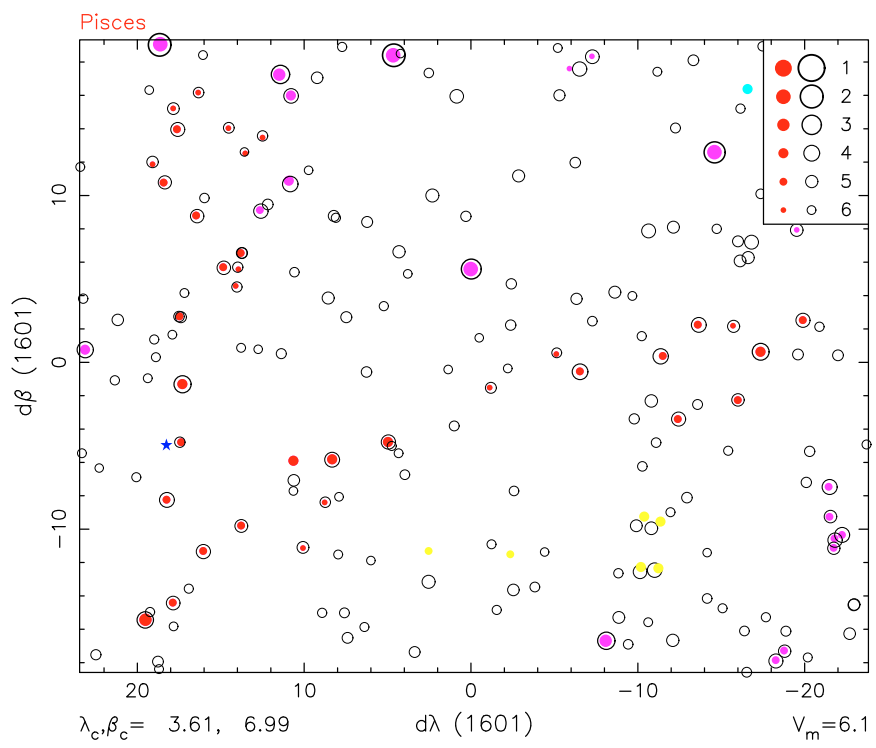

Fig. C.44. Pisces. The blue $\star$ indicates the position of K 801 according to Kepler, the emended position gives a much better coincidence with o Psc, about $3^{\circ}$ to the south.

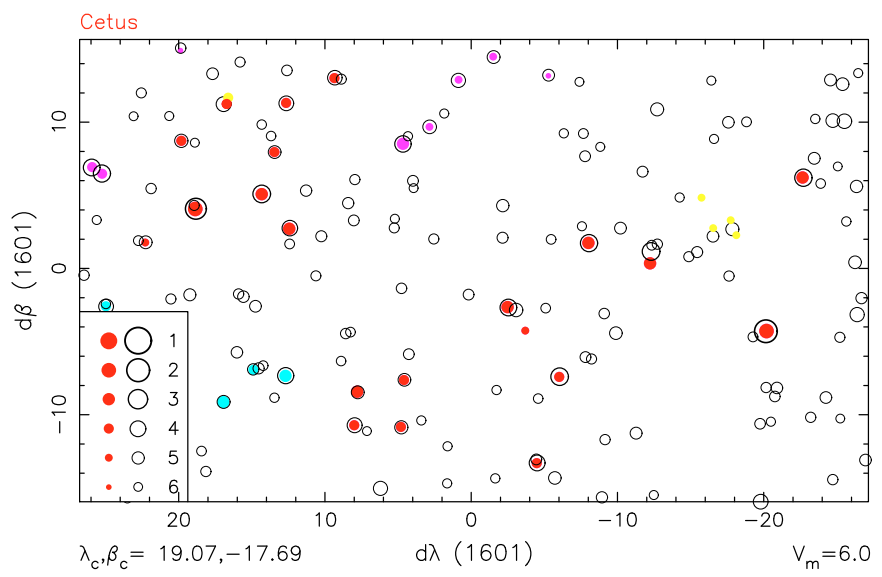

Fig. C.45. Cetus.

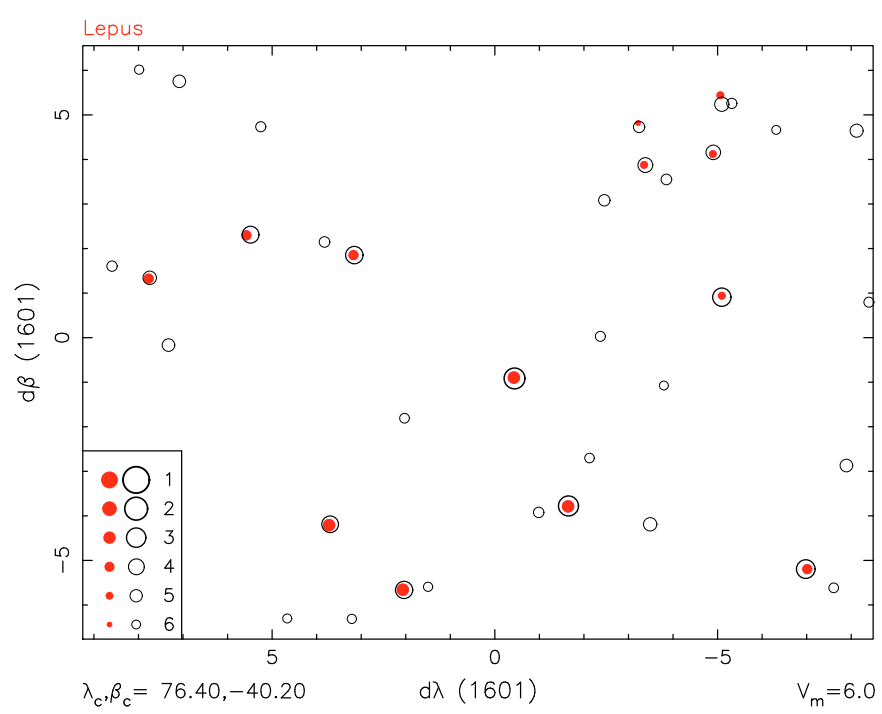

Fig. C.46. Lepus. 
A\&A 516, A28 (2010)

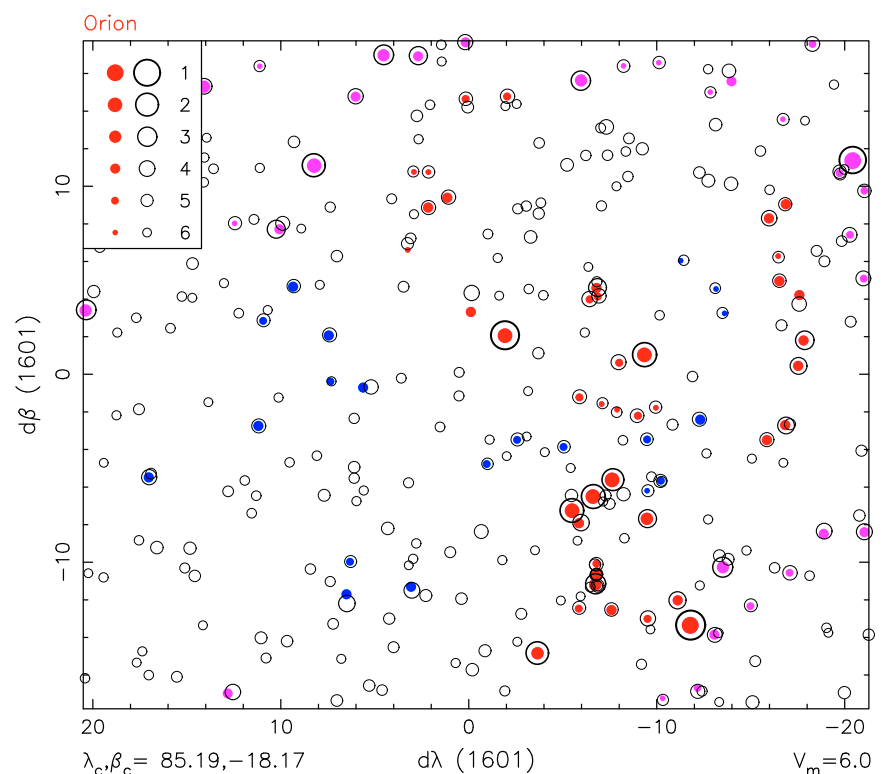

Fig. C.47. Orion.

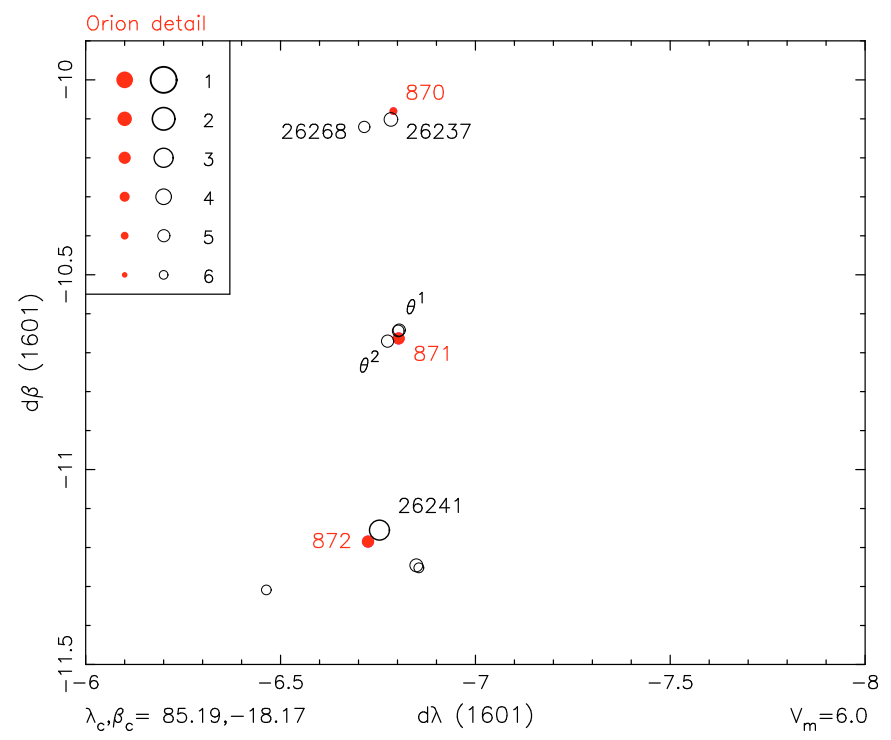

Fig. C.48. The sword of Orion.

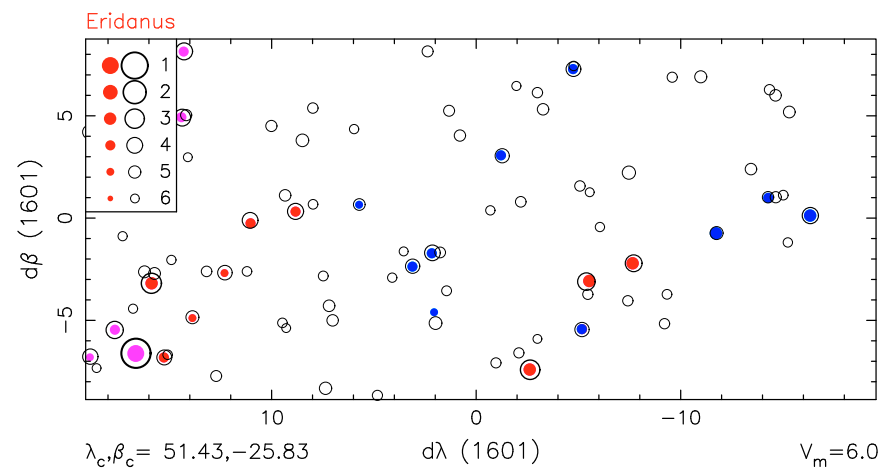

Fig. C.49. Eridanus.

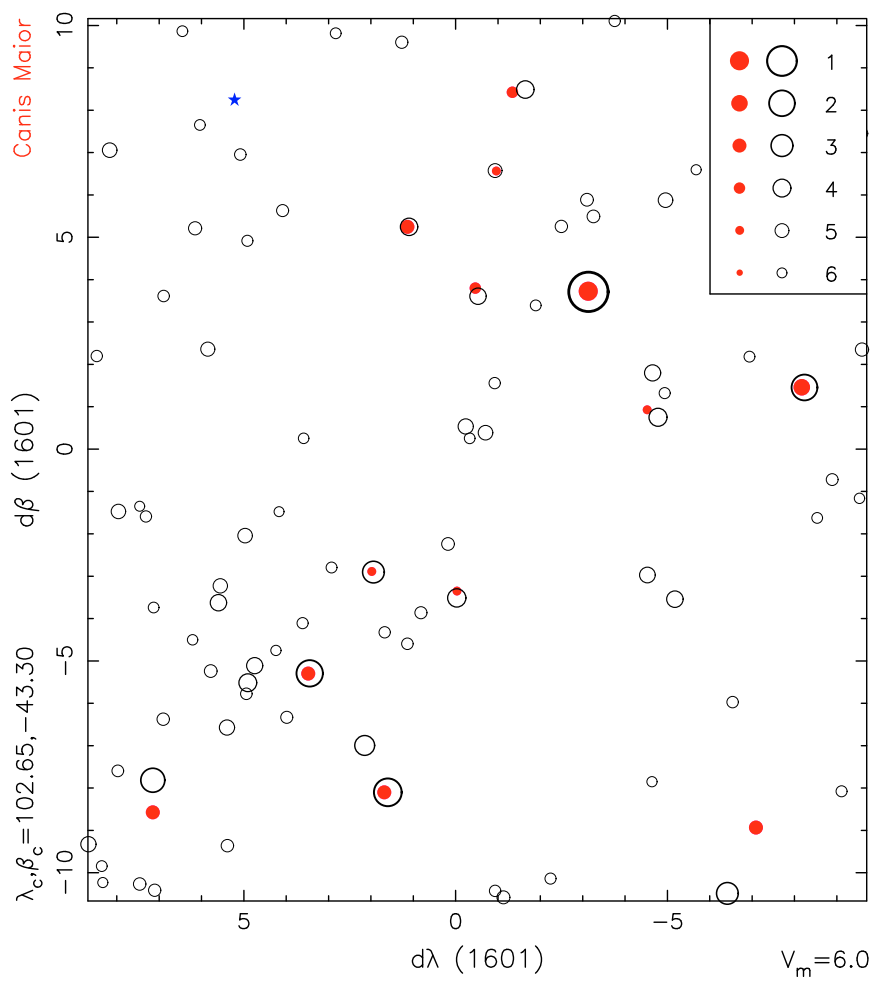

Fig. C.50. Canis Maior. The blue $\star$ indicates the postition of K 934 in Kepler.

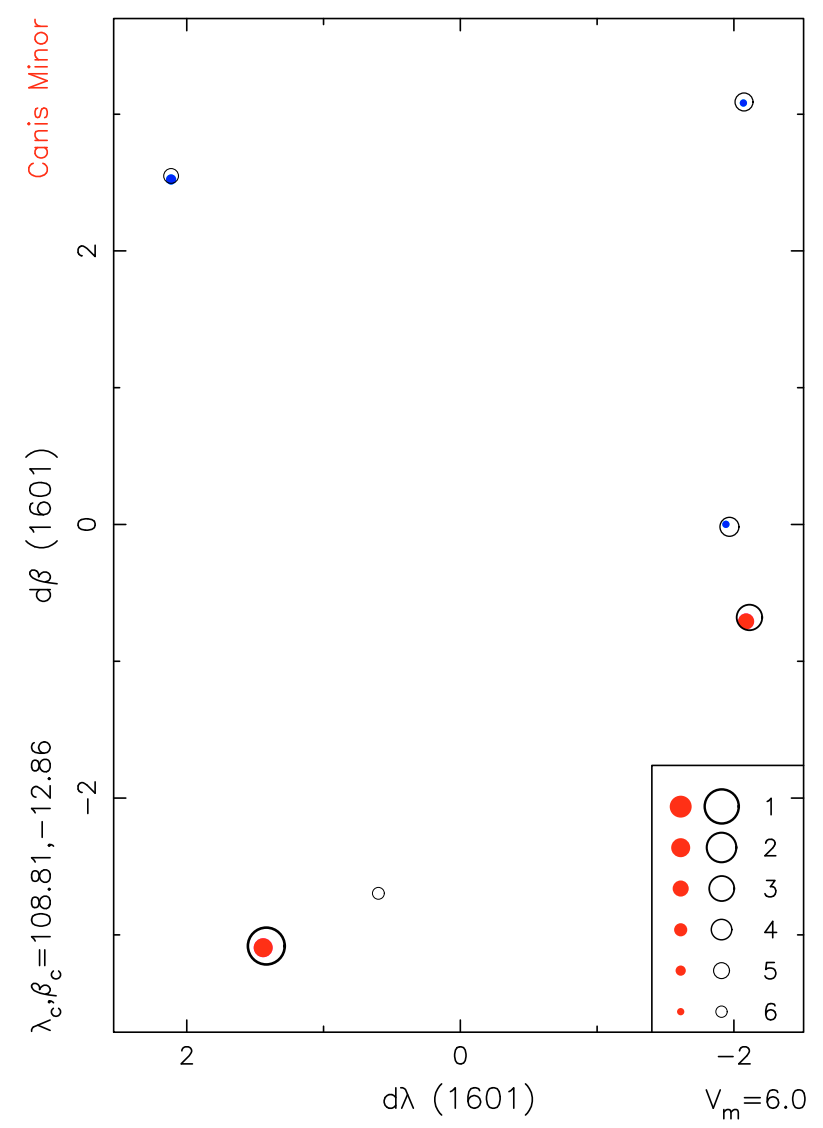

Fig. C.51. Canis Minor. 
F. Verbunt and R. H. van Gent: Three editions of the star catalogue of Tycho Brahe

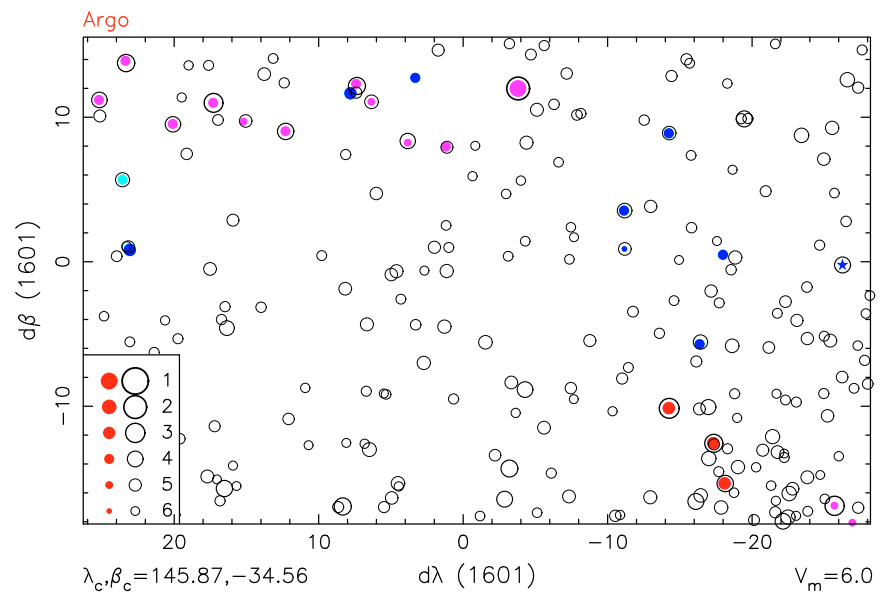

Fig. C.52. Argo. K 961 is near 23, 1 (see also Fig. C.54), its position as emended by Hevelius is indicated with the blue $\star$.

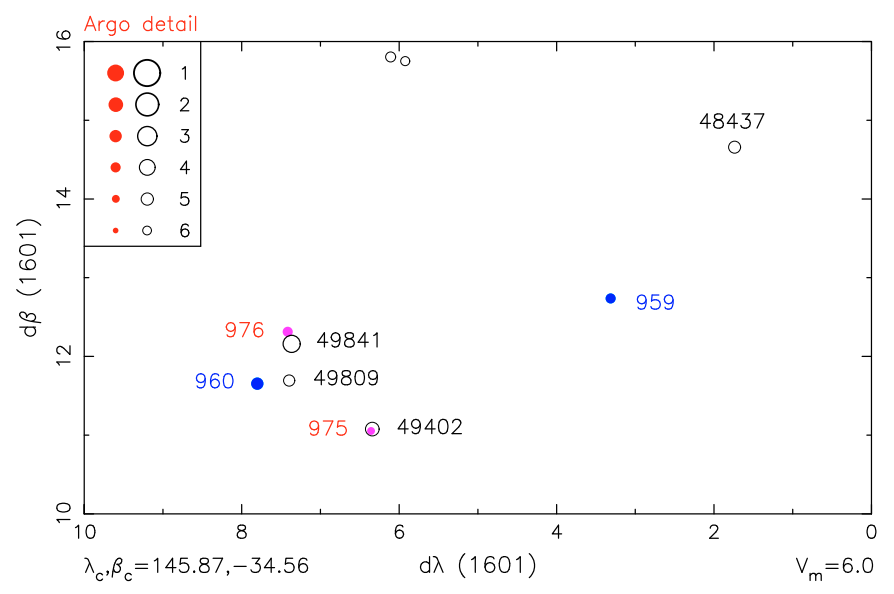

Fig. C.53. Argo detail.

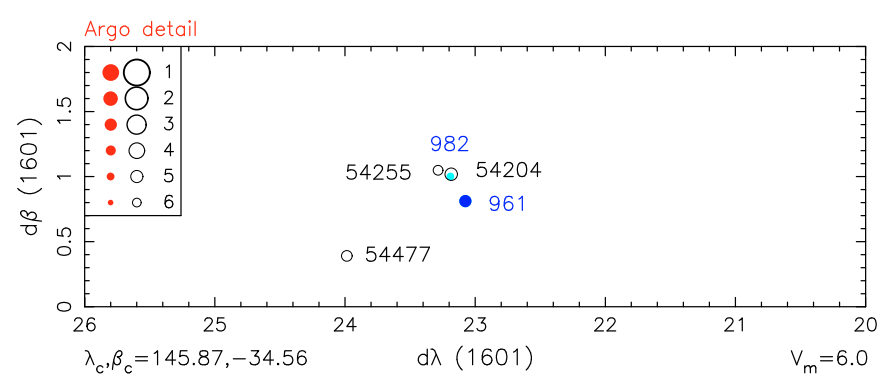

Fig. C.54. Argo detail.

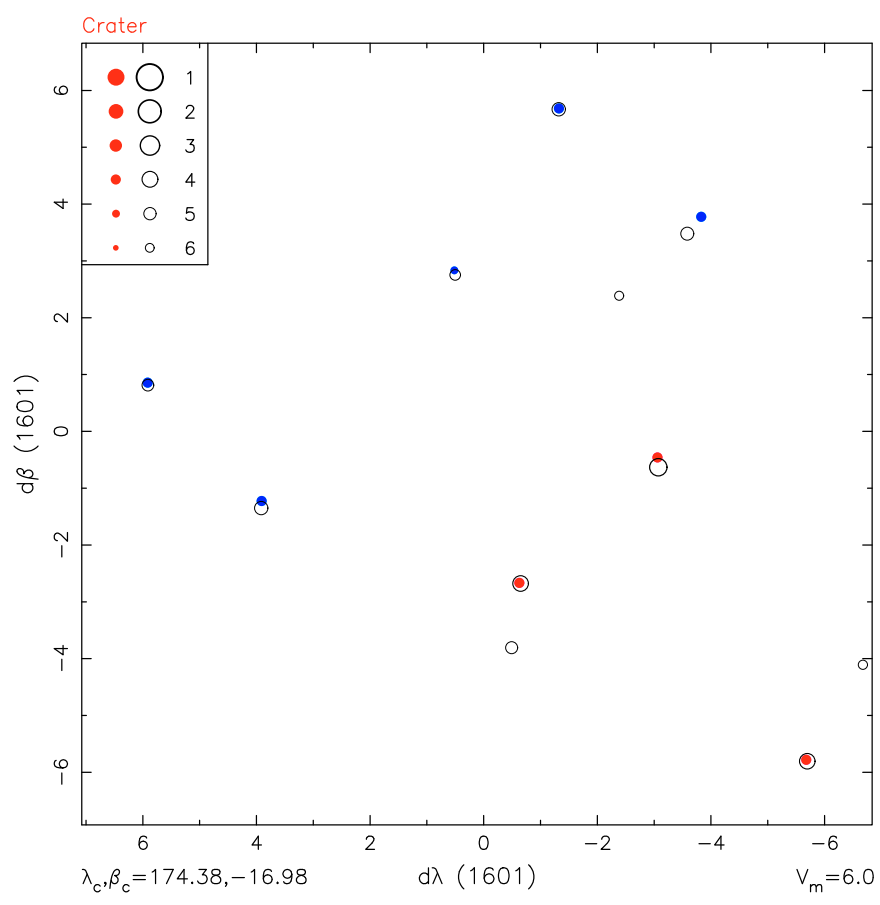

Fig. C.55. Crater.

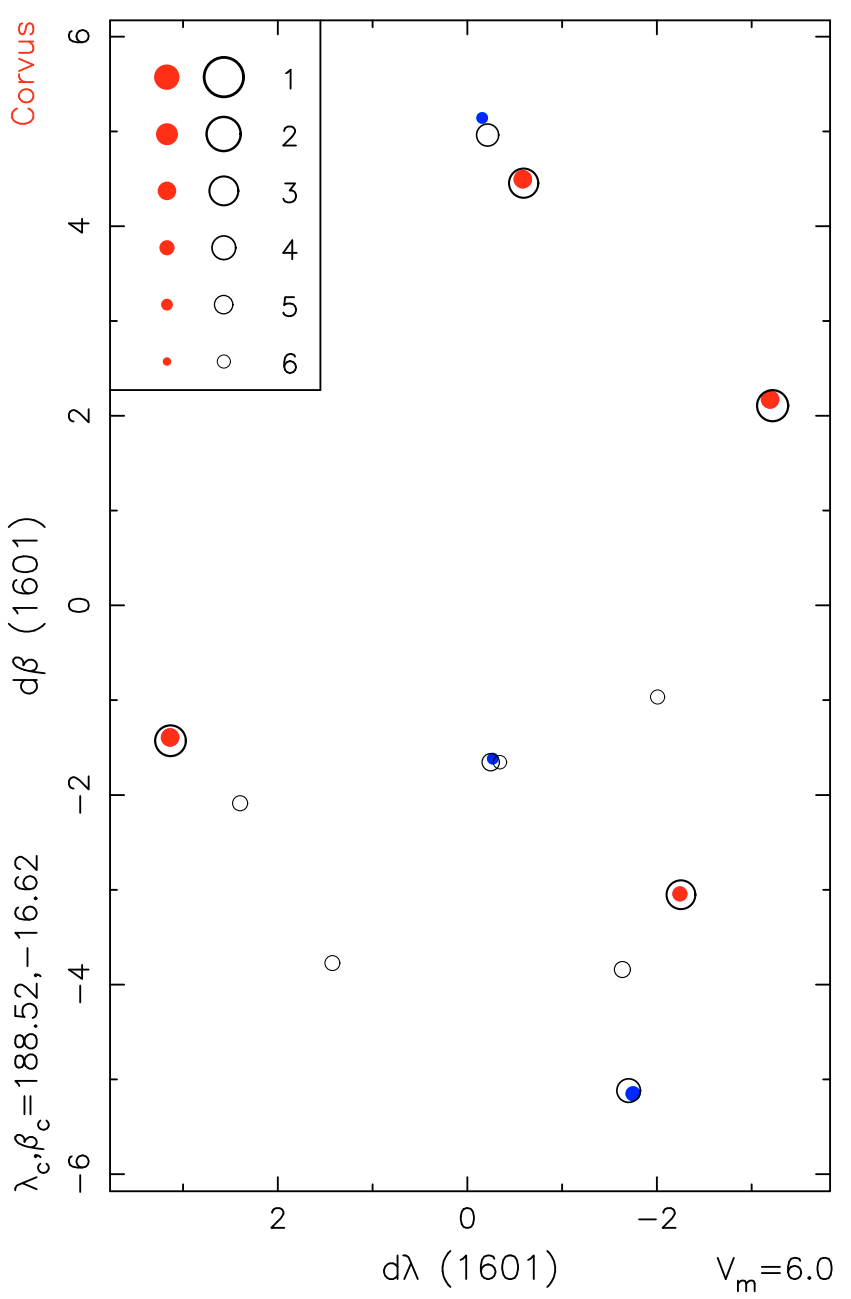

Fig. C.56. Corvus. 
A\&A 516, A28 (2010)

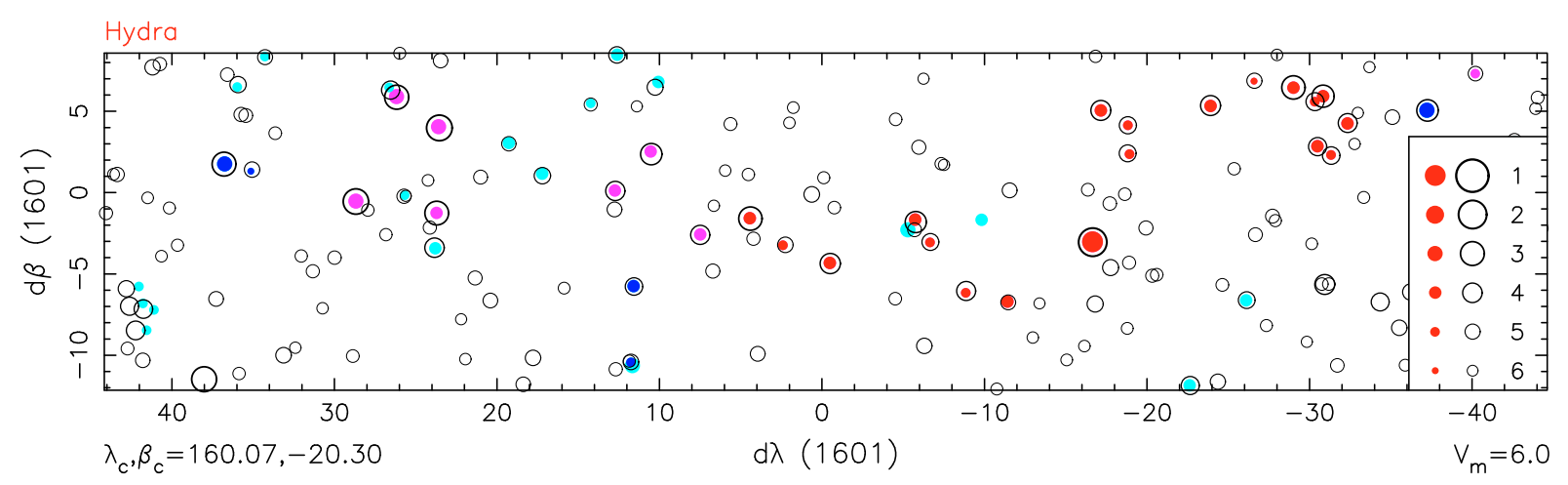

Fig. C.57. Hydra.

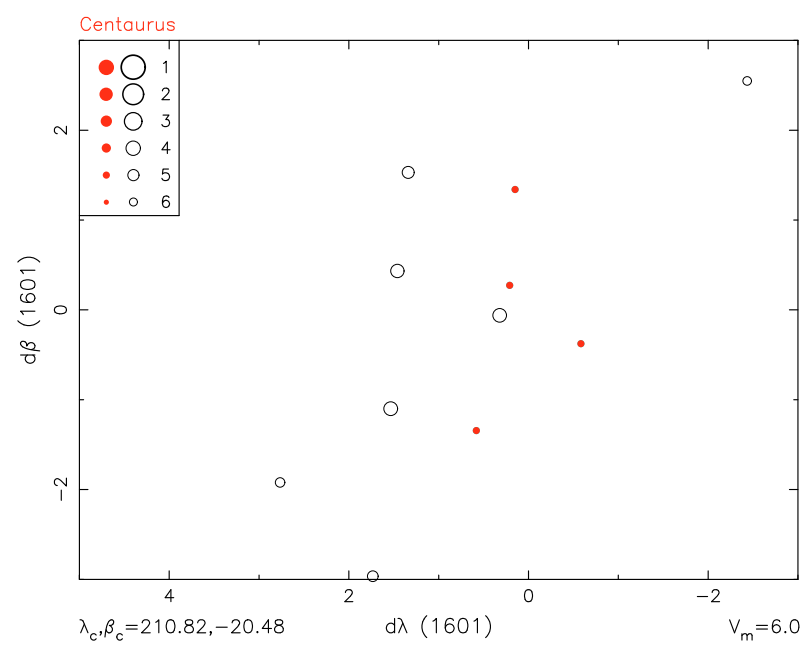

Fig. C.58. Centaurus. The catalogue longitudes appear shifted by about a degree. 